\title{
THE PROPERTIES OF MASSIVE, DENSE CLUMPS: MAPPING SURVEYS OF HCN AND CS
}

\author{
Jingwen Wu ${ }^{1,2}$, Neal J. Evans II ${ }^{3}$, Yancy L. ShiRley ${ }^{4}$, and Claudia Knez ${ }^{5}$ \\ ${ }^{1}$ Harvard-Smithsonian Center for Astrophysics, 60 Garden Street, MS78, Cambridge, MA 02138, USA; jwu@cfa.harvard.edu \\ 2 Jet Propulsion Laboratory, 4800 Oak Grove Drive, MS723, Pasadena, CA 91109, USA \\ ${ }^{3}$ Department of Astronomy, The University of Texas at Austin, 1 University Station, C1400, Austin, TX 78712, USA; nje@astro.as.utexas.edu \\ ${ }^{4}$ Steward Observatory, University of Arizona, Tucson, AZ 85721, USA; yshirley@as.arizona.edu \\ 5 Department of Astronomy, University of Maryland, College Park, MD 20742, USA \\ Received 2009 August 28; accepted 2010 March 1; published 2010 May 7
}

\begin{abstract}
We have mapped over 50 massive, dense clumps with four dense gas tracers: $\mathrm{HCN} J=1-0$ and $3-2$; and CS $J=2-1$ and 7-6 transitions. Spectral lines of optically thin $\mathrm{H}^{13} \mathrm{CN} 3-2$ and $\mathrm{C}^{34} \mathrm{~S} 5-4$ were also obtained toward the map centers. These maps usually demonstrate single well-peaked distributions at our resolution, even with higher $J$ transitions. The size, virial mass, surface density, and mean volume density within a well-defined angular size (FWHM) were calculated from the contour maps for each transition. We found that transitions with higher effective density usually trace the more compact, inner part of the clumps but have larger linewidths, leading to an inverse linewidth-size relation using different tracers. The mean surface densities are $0.29,0.33,0.78,1.09 \mathrm{~g} \mathrm{~cm}^{-2}$ within FWHM contours of CS 2-1, HCN 1-0, HCN 3-2, and CS 7-6, respectively. We find no correlation of $L_{\mathrm{IR}}$ with surface density and a possible inverse correlation with mean volume density, contrary to some theoretical expectations. Molecular line luminosities $L_{\text {mol }}^{\prime}$ were derived for each transition. We see no evidence in the data for the relation between $L_{\text {mol }}^{\prime}$ and mean density posited by modelers. The correlation between $L_{\text {mol }}^{\prime}$ and the virial mass is roughly linear for each dense gas tracer. No obvious correlation was found between the line luminosity ratio and infrared luminosity, bolometric temperature, or the $L_{\mathrm{IR}} / M_{\mathrm{Vir}}$ ratio. A nearly linear correlation was found between the infrared luminosity and the line luminosity of all dense gas tracers for these massive, dense clumps, with a lower cutoff in luminosity at $L_{\mathrm{IR}}=10^{4.5} L_{\odot}$. The $L_{\mathrm{IR}}-L_{\mathrm{HCN} 1-0}^{\prime}$ correlation agrees well with the one found in galaxies. These correlations indicate a constant star formation rate per unit mass from the scale of dense clumps to that of distant galaxies when the mass is measured for dense gas. These results support the suggestion that starburst galaxies may be understood as having a large fraction of gas in dense clumps.
\end{abstract}

Key words: galaxies: formation - ISM: clouds - ISM: molecules - stars: formation - submillimeter: ISM

Online-only material: color figures

\section{INTRODUCTION}

A full understanding of how stars form, both in the Milky Way and in other galaxies, requires an improved picture of the formation of massive stars. Most of the stars in our Galaxy form in regions of clustered star formation, and nearly all massive stars appear to form in dense clusters (Elmegreen 1985; Carpenter 2000; Lada \& Lada 2003). Massive forming stars provide the only obvious signposts of star formation in distant regions of our Galaxy and certainly in other galaxies. The star formation prescription most widely used in simulations of galaxy formation and evolution, the Kennicutt-Schmidt law (Schmidt 1959; Kennicutt 1998) is based on tracers like $\mathrm{H} \alpha$ (e.g., Calzetti 2008), which trace the formation only of relatively massive stars. The signs of formation of lower mass stars are mostly invisible at the distances of other galaxies. For all these reasons, a better understanding of the formation of massive stars is important.

However, our knowledge of the massive star formation process is still limited, in both observational and theoretical aspects, in contrast to the progress made in the study of lowmass star formation. Observational progress has been limited by distance, complexity, and the rapid evolution of young massive stars. Most high-mass star-forming regions are very distant from us, normally at least an order of magnitude farther than the nearby low-mass star-forming regions. When they are close enough to study in detail, such as the Orion molecular cloud, we find an extremely complex environment marked by high turbulence, high density and opacity (e.g., O'Dell 2005), and multiple large-scale motions, including possible explosive events. The fast evolution of the new-born massive stars leads to a harsh radiative environment. Together with the jets, winds, and outflows, the radiation field rapidly destroys the evidence that we need.

The confusing observational picture allows quite a variety of theoretical models to exist. Recently, the models have tended to fall into one of the two camps. Bonnell et al. (1997, 1998) and Bonnell \& Bate (2005) have begun with a clump containing many Jeans masses, which fragments rapidly into small stars that compete to accrete the remaining gas. The other camp, represented by McKee \& Tan (2002, 2003) and Tan et al. (2006), argue that such a clump will be composed of a spectrum of turbulent cores, essentially scaled-up versions of the well-studied cores that form low-mass stars. The higher mass stars then form because turbulent pressure produces a much higher accretion rate. For an interesting discussion of these different approaches, see Krumholz \& Bonnell (2007). Recent simulations including radiative feedback from forming protostars found that the extreme fragmentation found in earlier, isothermal simulations was greatly suppressed, allowing more massive stars to form (Krumholz et al. 2007; Urban et al. 2010; Krumholz et al. 2010). 
In this situation, one positive step to study massive star formation is to make statistical studies based on systematic surveys toward a large and well-characterized sample of massive starforming regions. Using suitable tracers, which can reveal the physical and dynamical properties of the regions, we can provide the basic constraints that successful theories must satisfy.

Star formation is not uniformly distributed over molecular clouds, but is restricted to regions within molecular clouds where the density exceeds some threshold, roughly $n>10^{4}$ or $10^{5} \mathrm{~cm}^{-3}$ (e.g., Evans 2008). There have been many detailed studies of individual regions of massive star formation and surveys of likely regions in tracers of dense gas or dust (e.g., Plume et al. 1992, 1997; Zinchenko et al. 1995; Anglada et al. 1996; Sridharan et al. 2002; Beuther et al. 2002; Wu \& Evans 2003). These surveys have mostly used signposts of massive star formation, such as masers, $\mathrm{H}$ II regions, or infrared sources with particular colors. Less biased surveys, using infrared dark clouds or blind surveys for millimeter wavelength dust continuum, are beginning to become available (e.g., Price et al. 2001; Williams et al. 2007; Schuller et al. 2009; Aguirre et al. 2009; Rosolowsky et al. 2009). These will demand follow-up studies, and the question arises of what can be learned from different tracers.

This work will focus on a large sample of massive starforming regions that are in an early phase of star formation, as indicated originally by the association with water masers. We have in the past referred to these regions as "cores," but McKee and co-workers prefer to restrict the use of "core" to a smaller region that will form a single or small star group, and they refer to regions that will form clusters as "clumps." The expectation is that star-forming clumps will contain multiple cores. We adopt the McKee terminology here and refer to these objects as clumps. We emphasize however, that their properties are very different from the objects usually going by this name. As described by Williams et al. (2000), clumps are traced by extinction or $\mathrm{C}^{18} \mathrm{O}$ peaks and have modest mean densities of $n \sim 10^{3}$. Most may not be gravitationally bound. The clumps we are studying here are very dense $\left(\langle n\rangle \sim 10^{6}\right.$, e.g., Plume et al. 1997), on average 100 times denser than the "cores" in lowmass star-forming regions (e.g., Mueller et al. 2002). To keep this distinction in mind, we refer to them as "dense clumps." Since most also have high masses, we often refer to them as "massive dense clumps."

The sample has been well studied by multiple CS line surveys (Plume et al. 1992, 1997). A subsample of these sources has been mapped in the CS 5-4 transition (Shirley et al. 2003) and in $350 \mu \mathrm{m}$ dust continuum emission (Mueller et al. 2002). Based on the dust continuum data, a one-dimensional model was constructed to determine the mass, bolometric luminosity, and density distribution of those massive dense clumps; a power-law density distribution $\left(n(r) \propto r^{-p}\right.$ with $\left.\langle p\rangle=1.8\right)$ was found to fit the data well (Mueller et al. 2002). The CS 5-4 maps have provided further constraints on the size and virial mass of the dense clumps (Shirley et al. 2003).

While the maps of CS 5-4 emission and dust continuum emission provided valuable statistical data on sizes and masses, systematic studies of other commonly used tracers can provide a valuable comparison set for surveys in these tracers, both in distant regions of our Galaxy and in other galaxies. In particular, $\mathrm{HCN}$ has been used much more widely in surveys of starburst galaxies, and we need a set of fiducial objects in order to understand those surveys.

Additional molecular lines that can trace dense clumps, as well as their maps, are needed for this purpose. We selected the CS 7-6, CS 2-1, HCN 3-2, and HCN 1-0 transitions for further mapping. CS 7-6 and CS 2-1 maps can constrain models of the clumps that have been studied with CS 5-4 at different excitation levels. HCN 3-2 is a possible indicator of infall in massive star-forming regions (Wu \& Evans 2003), and it may be able to constrain mass inflow rates needed in theories of massive star formation.

Recent work (Gao \& Solomon 2004a, 2004b) shows that the luminosity of $\mathrm{HCN} 1-0$ has a tight and linear correlation with infrared luminosity, for both nearby normal galaxies and distant starburst galaxies, suggesting that star formation efficiency may be constant in these different systems when measured against tracers of the dense gas. We can directly test this $L_{\mathrm{HCN}}^{\prime}-\mathrm{L}_{\mathrm{IR}}$ correlation in Galactic dense clumps, by mapping HCN 1-0 toward massive dense clumps. Our results, based on the data in this survey, did indeed suggest that the ratio of massive star formation to the massive dense gas is similar in Galactic dense clumps as in starburst galaxies (Wu et al. 2005). That result suggests that $\mathrm{HCN}$ can be used to connect star formation on scales ranging from individual dense clumps in our Galaxy to distant galaxies. Then we may be able to apply our knowledge of Galactic massive star formation obtained from CS and HCN studies to star formation in other galaxies.

For all these purposes, and for building a complete data set to constrain models of individual massive dense clumps, we have carried out mapping surveys using HCN $3-2,1-0$, CS $7-6$, and $2-1$. In this paper we report the basic results of the survey.

\section{OBSERVATIONS}

\subsection{The Sample}

The sample in the survey is a subset of the larger sample of dense clumps associated with $\mathrm{H}_{2} \mathrm{O}$ masers that have been surveyed with several CS transitions (Plume et al. 1992, 1997), most of which have been mapped with CS 5-4 and dust emission (Shirley et al. 2003; Mueller et al. 2002). Table 1 lists the information on sources that have been mapped in this survey. The sources mapped in CS 5-4 (Shirley et al. 2003) have virial masses within the nominal core radius $\left(R_{\mathrm{CS}}\right)$ ranging from $30 M_{\odot}$ to $2750 M_{\odot}$, with a mean of $920 M_{\odot}$. The sources in this category have infrared luminosities ranging from $10^{3}$ to $10^{7} L_{\odot}$ and most contain compact or ultracompact $\mathrm{H}_{\text {II }}$ (UCHII) regions. These dense clumps are mostly quite massive and have been well studied by several tracers; we will focus on these sources for statistical studies of the properties of massive dense clumps. To extend the sample toward lower luminosities for the study of $L_{\mathrm{HCN}}^{\prime}-\mathrm{L}_{\mathrm{IR}}$ correlation, we selected 14 IRAS sources from outflow surveys (Zhang et al. 2005; Wu et al. 2004) and a few lower mass clumps from other publications. These added sources are listed in part 2 of Table 1, separated by a line from the well-studied massive clumps in Table 1. These clumps have various luminosities; in this paper they are not included in our statistics of the properties of massive dense clumps.

We mapped massive clumps in the HCN 3-2, HCN 1-0, CS 7-6, and CS 2-1 transitions. Because of observing constraints, maps of a particular transition are not available for all sources in our sample, but we have attempted to map as many sources as possible in all the tracers. In addition to mapping the clumps in the main transitions, we also observed the optically thin isotopes of $\mathrm{HCN}$ and $\mathrm{CS}\left(\mathrm{H}^{13} \mathrm{CN} 3-2\right.$ and $\left.\mathrm{C}^{34} \mathrm{~S} 5-4\right)$ at the center position of most of these massive clumps to constrain models and to study infall in massive clumps. Observations were taken between 1996 and 2007, with information presented in Table 2. 
Table 1

Mapping Surveys Toward Massive Clumps ${ }^{\mathrm{a}}$

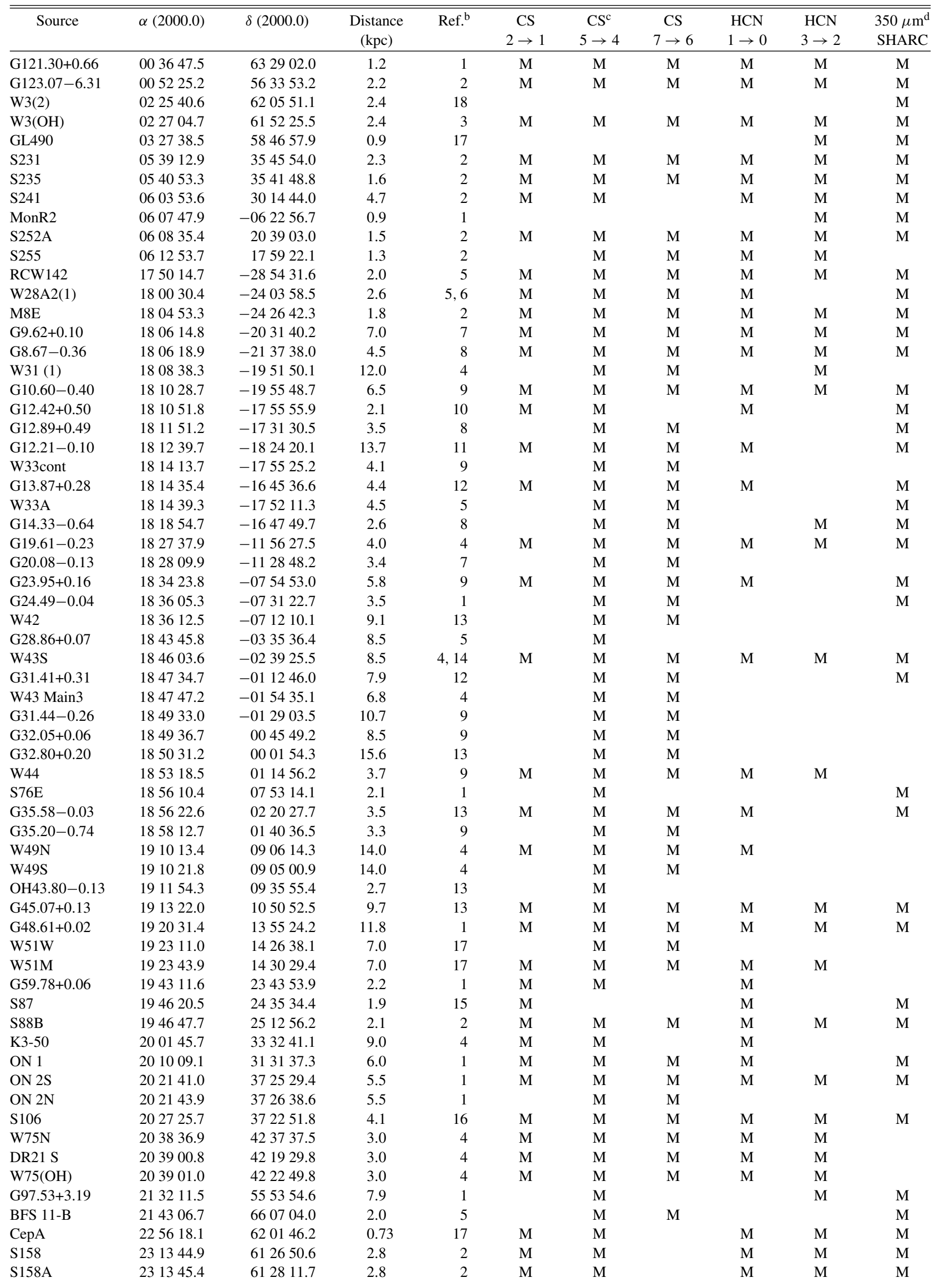


Table 1

(Continued)

\begin{tabular}{|c|c|c|c|c|c|c|c|c|c|c|}
\hline Source & $\alpha(2000.0)$ & $\delta(2000.0)$ & $\begin{array}{c}\text { Distance } \\
(\mathrm{kpc})\end{array}$ & Ref. ${ }^{b}$ & $\begin{array}{c}\text { CS } \\
2 \rightarrow 1\end{array}$ & $\begin{array}{c}\mathrm{CS}^{\mathrm{c}} \\
5 \rightarrow 4\end{array}$ & $\begin{array}{c}\text { CS } \\
7 \rightarrow 6\end{array}$ & $\begin{array}{c}\mathrm{HCN} \\
1 \rightarrow 0\end{array}$ & $\begin{array}{c}\mathrm{HCN} \\
3 \rightarrow 2\end{array}$ & $\begin{array}{l}350 \mu \mathrm{m}^{\mathrm{d}} \\
\text { SHARC }\end{array}$ \\
\hline NGC7538-IRS9 & 231401.7 & 612719.9 & 2.8 & 2 & M & & & M & M & M \\
\hline S157 & 231604.4 & 600140.6 & 2.5 & 2 & M & M & M & M & M & M \\
\hline IRAS19094+0944 & 191152.0 & 094946.2 & 6.11 & 19 & M & & & M & & \\
\hline IRAS20050+2720 & 200706.7 & 272852.9 & 0.73 & 19 & M & & & M & M & \\
\hline IRAS20106+3545 & 201231.3 & 355446.3 & 1.64 & 19 & M & & & M & & \\
\hline IRAS20188+3928 & 202039.4 & 393751.9 & 0.31 & 19 & M & & & M & M & \\
\hline IRAS20216+4107 & 202324.2 & 411739.8 & 1.70 & 20 & M & & & M & M & \\
\hline IRAS20220+3728 & 202355.7 & 373810.5 & 4.49 & 19 & M & & & M & & \\
\hline IRAS20333+4102 & 203509.6 & 411317.7 & 1.6 & 19 & M & & & M & M & \\
\hline IRAS21391+5802 & 214042.4 & 581609.6 & 0.75 & 19 & M & & & M & M & \\
\hline IRAS22172+5549 & 221909.0 & 560444.8 & 2.87 & 19 & M & & & M & & \\
\hline IRAS22198+6336 & 222127.6 & 635142.3 & 1.28 & 19 & M & & & M & & \\
\hline IRAS23385+6053 & 234053.2 & 611021.0 & 6.94 & 19 & M & & & M & & \\
\hline IRAS03282+3035 & 033121.0 & 304532.6 & 0.22 & 21 & M & & & M & M & \\
\hline L483 & 181729.8 & -043938.3 & 0.20 & 22 & & & & & M & \\
\hline L1251B & 223847.1 & 751128.8 & 0.30 & 23 & M & & & M & & \\
\hline
\end{tabular}

Notes.

a " $\mathrm{M}$ " in the table indicates that the source has been mapped in this transition.

b (1) $\mathrm{R}_{N}$ Plume et al. 1992; (2) Blitz et al. 1982; (3) Harris \& Wynn-Williams 1976; (4) Genzel \& Downes 1977; (5) Braz \& Epchtein 1983; (6) Chini et al. 1986; (7) Hofner et al. 1996; (8) Val'tts et al. 2000; (9) Solomon et al. 1987; (10) Zinchenko et al. 1994; (11) Hunter et al. 2000; (12) Churchwell et al. 1990; (13) Downes et al. 1980; (14) Wood \& Churchwell 1989; (15) Brand \& Blitz 1993; (16) Wink et al. 1982; (17) Zhou et al. 1996; (18) Plume et al. 1997; (19) Zhang et al. 2005; (20) Wu et al. 2004; (21) Cernis 1990; (22) Dame \& Thaddeus 1985; (23) Kun \& Prusti 1993.

c Mapping survey done by Shirley et al. (2003).

d Mapping survey done by Mueller et al. (2002).

\subsection{Observations with the FCRAO}

Maps of HCN 1-0 and CS 2-1 were made with the $14 \mathrm{~m}$ telescope of the Five College Radio Astronomy Observatory (FCRAO). The 16-element focal plane array (SEQUOIA) was used, with typical system temperatures 100-200 K. A velocity resolution of $0.1 \mathrm{~km} \mathrm{~s}^{-1}$ was achieved with the $25 \mathrm{MHz}$ bandwidth on the dual channel correlator (DCC). We converted the measured $T_{A}^{*}$ to $T_{R}$ via $T_{R}=T_{A}^{*} /\left(\eta_{\mathrm{FSS}} \eta_{c}\right)$, with $\eta_{\mathrm{FSS}}=0.7$. The value of $\eta_{c}$ depends on source size; for the typical map in this study $\left(\sim 10^{\prime}\right), \eta_{C}=0.7$. The map size was extended until the edge of the HCN $J=1 \rightarrow 0$ and CS $J=2 \rightarrow 1$ emission was reached, typically at the $2 \sigma$ level (mean $\sigma \sim 0.3 \mathrm{~K} \mathrm{~km} \mathrm{~s}^{-1}$ ), so we could obtain the total line luminosity.

\subsection{Observations with the CSO}

All the other observations were made at the $10.4 \mathrm{~m}$ telescope of the Caltech Submillimeter Observatory ${ }^{6}$ (CSO). The observing date, line frequency, beam size, and main beam efficiency of each line are listed in Table 2. The rest frequencies of HCN 3-2, $\mathrm{H}^{13} \mathrm{CN} 3-2$, and $\mathrm{C}^{34} \mathrm{~S} 5-4$ lines have been updated (Ahrens et al. 2002; Gottlieb et al. 2003) after some of our observations. We have corrected our data to the new frequencies listed in Table 2. The position switching mode was used; reference positions were checked when necessary. This is important for tracers that may show absorption features in the spectra (for example, HCN 3-2). We checked all the reference positions for HCN 3-2 emission and found them to be free from emission $\left(T_{A}^{*}<0.5 \mathrm{~K}\right)$. Pointing was checked periodically using planets and CO-bright stars. The pointing accuracy was usually better

\footnotetext{
6 The CSO is operated by the California Institute of Technology under funding from the National Science Foundation, contract AST 90-15755.
}

than 6" for all the runs. Map sizes of HCN 3-2 and CS 7-6 were extended to at least the point where the emission was one third of the peak strength, but usually maps extend to as low as one tenth of the peak strength (typically the $3 \sigma$ level, with the mean $\sigma \sim 1 \mathrm{~K} \mathrm{~km} \mathrm{~s}^{-1}$ ). The beam sizes and efficiencies were fairly constant for all transitions except for the CS 7-6, for which various different receiver optics were in place at different times. All data were scaled by the efficiency for the run on which they were taken to obtain the values of $T_{R}^{*}$ given in tables and used in the analysis.

\section{OBSERVATIONAL RESULTS}

Maps were obtained for 56 clumps with HCN 1-0, 43 clumps with HCN 3-2, 56 clumps with CS 2-1, and 51 clumps with CS $7-6$. The spectral line information at the central $(0,0)$ position of each map is listed in Tables 3-5.

\subsection{CS Results}

Table 3 presents the peak temperature of the line $\left(T_{R}^{*}\right)$, integrated intensity $\left(\int T_{R}^{*} \mathrm{~d} v\right)$, LSR velocity, and FWHM linewidth of CS 2-1, CS 7-6, and C ${ }^{34}$ S 5-4 transitions for massive dense clumps. Central position spectra of CS 2-1 and CS 7-6 have also been observed by Plume et al. $(1992,1997)$, who obtained CS 2-1 lines with the IRAM $30 \mathrm{~m}$ telescope at Pico Veleta, and CS 7-6 lines from the CSO. Comparing to the results in Plume et al. $(1992,1997)$, the observed parameters of CS 7-6 are consistent for the two CSO observations. The mean ratio of FWHM linewidth in the current observations to those from Plume's earlier data is $1.1 \pm 0.4$, with median ratio of 1.1. The mean ratio for $\int T_{R}^{*} \mathrm{~d} v$ is $1.1 \pm 0.7$, with a median ratio of 1.0 . For the results of CS 2-1 observations, the IRAM data give a 
Table 2

Observing Information

\begin{tabular}{|c|c|c|c|c|c|}
\hline Transitions & Dates & Telescope & $\begin{array}{c}\text { Frequency } \\
(\mathrm{GHz})\end{array}$ & $\begin{array}{c}\theta_{m b} \\
(\operatorname{arcsec})\end{array}$ & $\eta_{m b}$ \\
\hline \multirow[t]{6}{*}{ HCN 3-2 } & 2002 Jun & $\mathrm{CSO}$ & 265886.188 & 28.1 & 0.64 \\
\hline & 2002 Dec & $\mathrm{CSO}$ & 265886.188 & 28.1 & 0.60 \\
\hline & 2003 Jul & $\mathrm{CSO}$ & 265886.434 & 28.1 & 0.76 \\
\hline & 2004 Jan & $\mathrm{CSO}$ & 265886.434 & 28.1 & 0.66 \\
\hline & 2004 Jun & $\mathrm{CSO}$ & 265886.434 & 28.1 & 0.64 \\
\hline & 2007 Oct & $\mathrm{CSO}$ & 265886.434 & 28.1 & 0.77 \\
\hline \multirow[t]{7}{*}{ CS 5-4 } & 1996 Sep & $\mathrm{CSO}$ & 244935.610 & 24.5 & 0.56 \\
\hline & $1996 \mathrm{Dec}$ & $\mathrm{CSO}$ & 244935.610 & 24.5 & 0.54 \\
\hline & 1997 Apr & $\mathrm{CSO}$ & 244935.610 & 24.5 & 0.56 \\
\hline & 1997 Jun & $\mathrm{CSO}$ & 244935.610 & 24.5 & 0.58 \\
\hline & $1997 \mathrm{Dec}$ & $\mathrm{CSO}$ & 244935.610 & 24.5 & 0.56 \\
\hline & 1998 Jul & $\mathrm{CSO}$ & 244935.610 & 24.5 & 0.56 \\
\hline & 1999 Jul & $\mathrm{CSO}$ & 244935.610 & 24.5 & 0.60 \\
\hline \multirow[t]{5}{*}{ CS 7-6 } & 1998 Jul & $\mathrm{CSO}$ & 342882.900 & 16.9 & 0.59 \\
\hline & $1998 \mathrm{Dec}$ & $\mathrm{CSO}$ & 342882.900 & 20.5 & 0.61 \\
\hline & 2000 Jun & $\mathrm{CSO}$ & 342882.900 & 24.7 & 0.77 \\
\hline & $2000 \mathrm{Dec}$ & $\mathrm{CSO}$ & 342882.900 & 24.7 & 0.63 \\
\hline & 2002 Jun & $\mathrm{CSO}$ & 342882.900 & 24.7 & 0.53 \\
\hline \multirow[t]{3}{*}{$\mathrm{HCN} 1-0$} & 2004 Apr & FCRAO & 88631.847 & 58.0 & 0.50 \\
\hline & 2004 Dec & FCRAO & 88631.847 & 58.0 & 0.50 \\
\hline & $2005 \mathrm{Feb}$ & FCRAO & 88631.847 & 58.0 & 0.50 \\
\hline \multirow[t]{3}{*}{ CS 2-1 } & 2004 Apr & FCRAO & 97980.968 & 50.0 & 0.50 \\
\hline & 2004 Dec & FCRAO & 97980.968 & 50.0 & 0.50 \\
\hline & $2005 \mathrm{Feb}$ & FCRAO & 97980.968 & 50.0 & 0.50 \\
\hline \multirow[t]{5}{*}{$C^{34}$ S 5-4 } & $1998 \mathrm{Dec}$ & CSO & 241016.176 & 31.0 & 0.66 \\
\hline & $2001 \mathrm{Jul}$ & $\mathrm{CSO}$ & 241016.176 & 31.0 & 0.73 \\
\hline & 2002 Jan & $\mathrm{CSO}$ & 241016.176 & 31.0 & 0.53 \\
\hline & 2002 Jun & $\mathrm{CSO}$ & 241016.176 & 31.0 & 0.59 \\
\hline & 2005 Nov & $\mathrm{CSO}$ & 241016.089 & 31.0 & 0.80 \\
\hline \multirow[t]{4}{*}{$\mathrm{H}^{13} \mathrm{CN} 3-2$} & 2002 Dec & $\mathrm{CSO}$ & 259011.814 & 28.9 & 0.60 \\
\hline & 2003 May & $\mathrm{CSO}$ & 259011.814 & 28.9 & 0.58 \\
\hline & 2004 Jun & $\mathrm{CSO}$ & 259011.814 & 28.9 & 0.64 \\
\hline & 2006 Jun & $\mathrm{CSO}$ & 259011.814 & 28.9 & 0.80 \\
\hline
\end{tabular}

stronger $T_{R}^{*}$ and larger $\int T_{R}^{*} \mathrm{~d} v$; the ratio of $\int T_{R}^{*} \mathrm{~d} v$ for the IRAM data to current FCRAO data is $3.3 \pm 1.8$. This difference comes from the different telescope sizes and beam sizes of the IRAM and the FCRAO. But the linewidth from the two observations are close: the FWHM ratio of IRAM CS $2-1$ data to FCRAO data is $1.1 \pm 0.2$, and the median is 1.1.

\subsection{HCN Results}

Table 4 presents the line information for HCN 3-2 and its isotopologue $\mathrm{H}^{13} \mathrm{CN} 3-2$. The mean integrated intensity ratio of $\mathrm{HCN} 3-2$ to $\mathrm{H}^{13} \mathrm{CN} 3-2$ is $9.7 \pm 6.8$. This ratio is smaller than the usual ${ }^{12} \mathrm{C} /{ }^{13} \mathrm{C}$ isotopic abundance ratio, partly due to the large fraction of self-absorption features presented in HCN 3-2 lines. About half of the sources in the massive core sample show double peaks. The description of line profiles is in Table 4.

Table 5 lists the observational results for $\mathrm{HCN} 1-0$. HCN $1-0$ has three hyperfine components $(F=1-0, F=2-1, F=$ $1-1)$, separated by $-7.064 \mathrm{~km} \mathrm{~s}^{-1}(F=1-0$ to $F=2-1)$ and $4.842 \mathrm{~km} \mathrm{~s}^{-1}(F=1-1$ to $F=2-1)$. We tried to fit the spectra with three Gaussian components with the same linewidth. The peak temperatures of every component, the LSR velocity of the main component $(F=2-1)$, the linewidth, and the integrated intensity are presented in Table 5. Massive clumps usually have large linewidths; sometimes the linewidth is wider than the separation of hyperfine components, so that two or three of these components will overlap. Sometimes one or two hyperfine
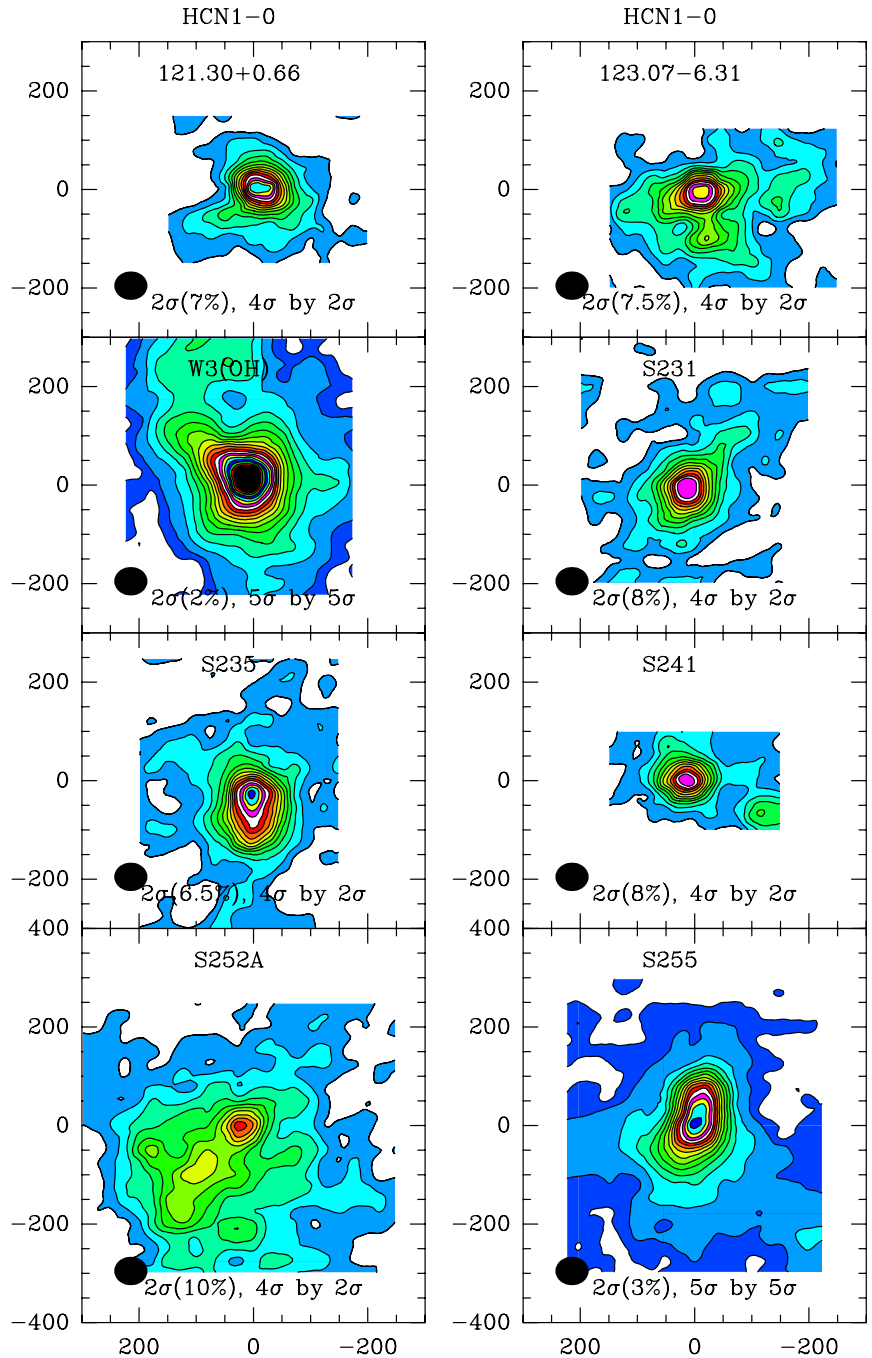

Figure 1. HCN 1-0 contour maps of massive clumps. The lowest contour level and increasing step of contours are indicated in the plot. The beam size is shown at the lower left of each map.

(A color version of this figure is available in the online journal.)

components are optically thick enough to show absorption. We have noted these features in Table 5. If the HCN 1-0 line is optically thin and the hyperfine levels are populated according to LTE, the line ratios between the three hyperfine components are $I(F=1-0): I(F=2-1): I(F=1-1)=1: 5: 3$. The observed ratios for most of our clumps (Table 5) do not agree with these optically thin ratios, as expected for lines that are almost surely optically thick. This hyperfine anomaly has also been reported in other massive star-forming regions (e.g., Pirogov et al. 1996; Pirogov 1999).

\subsection{Morphology and Multiplicity}

Contour maps of HCN 1-0, HCN 3-2, CS 2-1, and CS 7-6 are presented in Figures 1-23. These maps show a variety of morphologies, with some multiple peaks. HCN $1-0$ and CS $2-1$ maps cover a much larger area (typically greater than $7^{\prime} \times 7^{\prime}$ ) than the higher $J$ transition maps. A substantial fraction of these low- $J$ transition maps show multiple peaks. For HCN 1-0, 16 out of 53 maps (30\%) show multiple peaks; For CS 2-1, 21 out of 53 maps show multiple peaks (40\%). This ratio is lower for high- $J$ HCN and CS tracers. Only eight out of $42 \mathrm{HCN} 3-2$ maps (19\%), and one of 51 CS 7-6 (2\%) present multiple peaks. For CS 5-4 maps (Shirley et al. 2003), this ratio is $16 \%$ (nine 
Table 3

Observational Results of CS and $\mathrm{C}^{34}$ Transitions

\begin{tabular}{|c|c|c|c|c|c|c|c|c|c|c|c|c|}
\hline \multirow[t]{2}{*}{ Source } & \multicolumn{4}{|c|}{$\mathrm{CS} 2 \rightarrow 1$} & \multicolumn{4}{|c|}{$\mathrm{CS} 7 \rightarrow 6$} & \multicolumn{4}{|c|}{$\mathrm{C}^{34} \mathrm{~S} 5 \rightarrow 4$} \\
\hline & $\begin{array}{c}T_{R}^{*} \\
(\mathrm{~K}) \\
\end{array}$ & $\begin{array}{c}\int T_{R}^{*} \mathrm{~d} v \\
\left(\mathrm{~K} \mathrm{~km} \mathrm{~s}^{-1}\right)\end{array}$ & $\begin{array}{c}V_{\mathrm{LSR}} \\
\left(\mathrm{km} \mathrm{s}^{-1}\right)\end{array}$ & $\begin{array}{c}\text { FWHM } \\
\left(\mathrm{km} \mathrm{s}^{-1}\right)\end{array}$ & $\begin{array}{c}T_{R}^{*} \\
(\mathrm{~K}) \\
\end{array}$ & $\begin{array}{c}\int T_{R}^{*} \mathrm{~d} v \\
\left(\mathrm{~K} \mathrm{~km} \mathrm{~s}^{-1}\right)\end{array}$ & $\begin{array}{c}V_{\mathrm{LSR}} \\
\left(\mathrm{km} \mathrm{s}^{-1}\right)\end{array}$ & $\begin{array}{c}\text { FWHM } \\
\left(\mathrm{km} \mathrm{s}^{-1}\right)\end{array}$ & $\begin{array}{c}T_{R}^{*} \\
(\mathrm{~K}) \\
\end{array}$ & $\begin{array}{c}\int T_{R}^{*} \mathrm{~d} v \\
\left(\mathrm{~K} \mathrm{~km} \mathrm{~s}^{-1}\right)\end{array}$ & $\begin{array}{c}V_{\mathrm{LSR}} \\
\left(\mathrm{km} \mathrm{s}^{-1}\right)\end{array}$ & $\begin{array}{l}\text { FWHM } \\
\left(\mathrm{km} \mathrm{s}^{-1}\right)\end{array}$ \\
\hline G121.30+0.66 & $4.2(0.2)$ & $18.9(0.8)$ & $-17.87(03)$ & $3.82(09)$ & $3.1(0.4)$ & $9.0(1.1)$ & $-17.44(05)$ & $2.58(13)$ & $0.2(.08)$ & $0.6(0.2)$ & $-17.64(17)$ & $2.87(37)$ \\
\hline G123.07-6.31 & $3.6(0.3)$ & $13.9(1.0)$ & $-30.56(04)$ & $3.56(10)$ & $2.6(0.5)$ & $14.3(1.6)$ & $-30.97(09)$ & $5.33(21)$ & $0.2(.15)$ & $1.3(0.4)$ & $-30.29(37)$ & $5.35(108)$ \\
\hline $\mathrm{G} 135.28+2.80$ & $\ldots$ & $\ldots$ & $\ldots$ & $\ldots$ & $0.9(0.3)$ & $2.8(0.8)$ & $-71.49(09)$ & $2.47(26)$ & 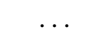 & $\ldots$ & $\ldots$ & $\ldots$ \\
\hline $\mathrm{W} 3(\mathrm{OH})$ & $6.7(0.1)$ & $36.0(0.6)$ & $-46.80(01)$ & $5.02(03)$ & $8.1(0.7)$ & $50.9(2.4)$ & $-47.65(04)$ & $6.05(10)$ & $1.0(0.1)$ & $5.9(0.5)$ & $-47.49(08)$ & $5.50(20)$ \\
\hline S231 & $3.6(0.4)$ & $18.4(1.5)$ & $-16.74(06)$ & $4.76(16)$ & $3.5(0.4)$ & $17.3(1.5)$ & $-15.82(04)$ & $4.38(11)$ & $0.5(.08)$ & $1.2(0.2)$ & $-15.77(07)$ & $2.48(15)$ \\
\hline S235 & $4.1(0.2)$ & $10.9(0.8)$ & $-16.78(02)$ & $2.33(06)$ & $5.0(0.2)$ & $19.5(0.8)$ & $-17.09(02)$ & $3.38(04)$ & $0.8(0.2)$ & $2.0(0.5)$ & $-16.93(09)$ & $2.35(24)$ \\
\hline $\mathrm{S} 241$ & $2.5(0.1)$ & $7.3(0.4)$ & $-8.74(02)$ & $2.63(05)$ & $0.6(0.2)$ & $1.9(0.5)$ & $-8.79(11)$ & $2.98(24)$ & $\ldots$ & $\ldots$ & $\ldots$ & $\ldots$ \\
\hline $\mathrm{S} 252 \mathrm{~A}$ & $3.8(0.2)$ & $12.8(0.8)$ & $9.05(03)$ & $3.09(07)$ & $2.7(0.3)$ & $10.5(0.9)$ & $9.63(05)$ & $3.57(11)$ & $\ldots$ & $\ldots$ & $\ldots$ & $\ldots$ \\
\hline $\mathrm{S} 255$ & $\ldots$ & $\ldots$ & $\ldots$ & $\ldots$ & $7.2(0.4)$ & $32.6(1.2)$ & $7.52(02)$ & $4.03(05)$ & $1.1(0.2)$ & $3.9(0.7)$ & 7.34(09) & $3.03(25)$ \\
\hline RCW142 & $7.3(0.3)$ & $44.2(1.4)$ & $17.08(03)$ & $5.23(08)$ & $10.6(0.5)$ & $83.0(2.3)$ & $16.63(03)$ & $6.81(07)$ & $4.3(0.4)$ & $26.5(1.4)$ & $17.35(05)$ & $5.60(12)$ \\
\hline W28A2(1) & $5.4(0.3)$ & $30.1(1.1)$ & $9.38(03)$ & 4.77(09) & $12.5(0.4)$ & $131.5(2.4)$ & $10.40(03)$ & $9.29(09$ & $3.4(0.2)$ & $23.4(0.7)$ & $9.77(03)$ & $5.91(09)$ \\
\hline M8 E & $4.3(0.3)$ & $10.4(0.8)$ & $10.58(03)$ & $2.15(08)$ & $5.3(0.4)$ & 18.4(1.2) & $10.91(03)$ & $3.29(07)$ & $1.9(0.2)$ & $4.6(0.4)$ & 10.81 & $2.23(08)$ \\
\hline G9.62+0.10 & $2.3(0.5)$ & $17.9(2.4)$ & $4.72(17)$ & $7.27(43)$ & $5.9(0.4)$ & $48.9(2.2)$ & $5.42(04)$ & 7.42(11) & $1.8(0.1)$ & $15.7(0.8)$ & $4.28(06)$ & $6.73(17)$ \\
\hline G8.67-0.36 & $1.7(0.3)$ & $14.3(1.1)$ & $35.23(13)$ & $8.12(32)$ & $4.6(0.3)$ & $30.1(1.5)$ & $34.97(04)$ & $5.74(10)$ & $1.0(0.2)$ & $5.7(0.5)$ & 34.84 & $5.08(23)$ \\
\hline W31 (1) & $\ldots$ & $\ldots$ & $\ldots$ & $\ldots$ & $3.0(0.9)$ & $19.9(3.2)$ & $65.00(18)$ & $5.84(43)$ & $0.9(0.1)$ & $8.0(0.6)$ & 66.27 & $8.56(29)$ \\
\hline G10.60-0.40 & $4.2(0.3)$ & $31.3(1.5)$ & $-3.24(06)$ & $6.67(14)$ & $13.0(0.5)$ & $131.3(2.5)$ & $-3.56(03)$ & $9.17(07)$ & $4.1(0.2)$ & $29.8(0.8)$ & $-2.86(03)$ & $6.72(08)$ \\
\hline $\mathrm{G} 12.42+0.50$ & $2.4(0.3)$ & $11.4(1.1)$ & $18.18(07)$ & $4.32(18)$ & $\ldots$ & $\ldots$ & $\ldots$ & $\ldots$ & $\ldots$ & $\ldots$ & $\ldots$ & $\ldots$ \\
\hline G12.89+0.49 & $1.5(0.4)$ & 8.1(1.6) & $33.77(16)$ & $4.39(44)$ & $3.9(0.3)$ & 21.1(1.1) & $33.18(04)$ & $5.09(09)$ & $1.3(0.1)$ & $5.2(0.3)$ & $33.22(03)$ & $3.78(08)$ \\
\hline G12.21-0.10 & $1.0(0.2)$ & $7.3(0.7)$ & $24.92(12)$ & $7.16(28)$ & $2.4(0.3)$ & $18.8(1.5)$ & $23.72(08)$ & 7.63(19) & 0.6 & $4.1(0.3)$ & 24.44 & $7.06(18)$ \\
\hline W33cont & $\ldots$ & $\ldots$ & $\ldots$ & $\cdots$ & $8.4(0.4)$ & $69.5(1.5)$ & $35.45(02)$ & $7.79(06)$ & $3.8(0.1)$ & $21.2(0.5)$ & $35.25(02)$ & $5.13(05)$ \\
\hline $\mathrm{G} 13.87+0.28$ & $11.2(0.4)$ & $76.3(1.8)$ & $\ldots$ & $6.38(06)$ & $4.6(0.4)$ & $14.4(1.1)$ & $48.32(03)$ & $2.57(07)$ & $0.9(0.1)$ & $2.5(0$ & 48.57 & $2.50(17)$ \\
\hline W33A & $1.4(0.3)$ & $7.8(1.4)$ & $36.58(16)$ & $5.59(36)$ & $1.6(0.5)$ & $10.3(1.4)$ & 37.59 & & $0.7(0.1)$ & $2.4(0$ & 37.89 & $3.22(24)$ \\
\hline G14.33-0.64 & $1.7(0.3)$ & $10.7(1.2)$ & $22.66(12)$ & $5.51(33)$ & $6.6(0.6)$ & $34.7(2.3)$ & $22.16(04)$ & $4.78(11)$ & $1.5(0.1)$ & $4.4(0.3)$ & $22.19(03)$ & $2.74(08)$ \\
\hline G19.61-0.23 & $0.6(0.2)$ & $4.0(0.7)$ & $42.67(24)$ & $5.61(62)$ & $3.1(0.8)$ & $31.1(3.5)$ & 42.63 & $9.72(3$ & $\ldots$ & $\ldots$ & $\ldots$ & $\ldots$ \\
\hline G20.08-0.13 & $\ldots$ & $\ldots$ & $\ldots$ & $\ldots$ & $3.0(0.3)$ & 29.1(1.6) & $41.74(06)$ & $8.83(17)$ & $\cdots$ & $\ldots$ & $\ldots$ & $\ldots$ \\
\hline $\mathrm{G} 23.95+0.16$ & $3.4(0.2)$ & $10.4(0.8)$ & 79.54(03) & $2.65(09)$ & $2.9(0.3)$ & $8.5(0.9)$ & $80.57(05)$ & $2.74(11)$ & $1.2(0.3)$ & $2.9(0.6)$ & $80.26(09)$ & $2.39(22)$ \\
\hline G24.49-0.04 & $1.3(0.2)$ & $7.3(0.7)$ & $110.17(08)$ & $4.57(23)$ & $1.3(0.3)$ & $4.4(1.0)$ & 109.60 & $6.14(38)$ & & $\ldots$ & $\ldots$ & $\ldots$ \\
\hline W42 & $\ldots$ & $\ldots$ & $\ldots$ & $\ldots$ & & $36.5(2.3)$ & $110.90(07)$ & & & $\ldots$ & $\ldots$ & $\ldots$ \\
\hline W43S & $5.5(0.1)$ & $28.2(0.7)$ & $97.51(02)$ & $4.38(05)$ & $7.2(0.7)$ & $41.0(2.6)$ & $97.76(05)$ & $5.13(11)$ & $1.4(0.1)$ & $5.9(0.1)$ & $98.00(02)$ & $3.97(04)$ \\
\hline $\mathrm{G} 31.41+0.31$ & $\ldots$ & $\ldots$ & $\ldots$ & $\ldots$ & $4.6(0.3)$ & $37.4(1.4)$ & $96.22(04)$ & $7.27(11)$ & $1.2(0.1)$ & $7.8(0.5)$ & $96.97(06)$ & $5.86(15)$ \\
\hline W43 Main3 & $\ldots$ & $\ldots$ & $\ldots$ & $\ldots$ & $2.3(0.4)$ & $18.8(1.8)$ & $98.36(10)$ & & & $\ldots$ & $\ldots$ & $\ldots$ \\
\hline G31.44-0.26 & $\ldots$ & $\ldots$ & $\ldots$ & $\ldots$ & & $12.9(0.8)$ & & & $0.6(.14)$ & $2.2(0.4)$ & $87.07(16)$ & $3.80(36)$ \\
\hline $\mathrm{G} 32.05+0.06$ & $\ldots$ & $\ldots$ & $\ldots$ & $\ldots$ & $1.5(0.3)$ & $11.7(1.1)$ & $95.50(10)$ & $7.26(22)$ & $0.3(.11)$ & $1.2(0.3)$ & $95.71(22)$ & $6.10(66)$ \\
\hline G32.80+0.20 & $\ldots$ & $\ldots$ & $\ldots$ & $\ldots$ & $4.4(0.6)$ & $38.8(2.7)$ & $14.22(08)$ & $8.40(18)$ & $\ldots$ & $\ldots$ & $\ldots$ & $\ldots$ \\
\hline W44 & $7.0(0.2)$ & $32.8(0.7)$ & $57.62(02)$ & $4.12(04)$ & $11.8(0.4)$ & $74.9(1.9)$ & $57.49(02)$ & $5.68(06)$ & $4.3(0.2)$ & $22.1(0.6)$ & $58.24(02)$ & $4.03(08)^{*}$ \\
\hline G35.58-0.03 & $0.8(0.2)$ & $4.5(0.6)$ & $53.22(12)$ & $5.11(29)$ & $3.0(0.5)$ & $19.7(2.3)$ & $53.22(08)$ & $5.72(23)$ & $0.3(.16)$ & $2.0(0.7)$ & 53.95 & $5.88(82)$ \\
\hline G35.20-0.74 & & $\ldots$ & $\ldots$ & $\ldots$ & $3.6(0.8)$ & $28.1(3.5)$ & $34.69(13)$ & $7.12(30)$ & $0.5(0.1)$ & $2.4(0.3)$ & $33.71(10)$ & $4.70(26)$ \\
\hline \multirow[t]{2}{*}{$\mathrm{W} 49 \mathrm{~N}$} & $2.1(0.2)$ & $40.2(0.9)^{*}$ & $2.54(11)^{*}$ & $7.51(23)$ & $8.9(0.6)^{*}$ & $130.7(3.2)$ & $6.81(05)$ & $13.88(11)$ & $0.5(0.1)$ & $3.6(0.4)$ & $3.18(18)$ & $6.41(39)$ \\
\hline & $2.8(0.2)$ & $\ldots$ & $12.00(08)$ & 7.78(19) & $\ldots$ & $\ldots$ & $\ldots$ & $\ldots$ & $0.6(0.1)$ & $4.7(0.4)$ & $12.36(19)$ & $7.86(43)$ \\
\hline W49S & $\ldots$ & $\ldots$ & $\ldots$ & $\ldots$ & $2.8(0.4)$ & $23.2(1.5)$ & $15.42(08)$ & $7.78(18)$ & $0.2(.09)$ & $1.9(0.3)$ & $14.82(23)$ & $7.56(54)$ \\
\hline G45.07+0.13 & $2.0(0.2)$ & $12.2(1.1)$ & $59.05(07)$ & $5.13(21)$ & $5.7(0.6)$ & $43.1(2.8)$ & $59.28(06)$ & & $1.2(0.2)$ & $8.7(0.6)$ & $59.27(08)$ & $6.33(23)$ \\
\hline G48.61+0.02 & $2.0(0.1)$ & $9.3(0.5)$ & $18.45(04)$ & $4.08(11)$ & $1.8(0.3)$ & $12.0(1.4)$ & $18.00(08)$ & $6.00(24)$ & $0.2(.06)$ & $0.5(.15)$ & $18.13(11)$ & $2.34(21)$ \\
\hline W51W & $\ldots$ & $\ldots$ & $\ldots$ & $\ldots$ & $5.2(0.6)$ & $23.6(2.0)$ & $51.04(05)$ & $4.12(12)$ & $0.9(0.2)$ & $3.5(0.4)$ & 51.01 & $3.39(25)$ \\
\hline W51M & $6.3(0.2)$ & $71.8(1.1)$ & $56.82(03)$ & $10.57(06)$ & $13.1(0.4)$ & $149.6(2.3)$ & $56.29(02)$ & $10.69(06)$ & $2.8(0.3)$ & $27.4(1.3)$ & $56.99(07)$ & $8.96(17)$ \\
\hline G59.78+0.06 & $2.6(0.2)$ & $7.2(0.7)$ & $22.78(04)$ & $2.36(10)$ & & $\ldots$ & $\ldots$ & $\ldots$ & $0.5(.13)$ & $1.2(0.3)$ & $22.74(10)$ & $2.22(31)$ \\
\hline S87 & $4.1(0.2)$ & $13.9(0.6)$ & $23.21(02)$ & $3.17(06)$ & $\ldots$ & $\ldots$ & $\ldots$ & $\ldots$ & $0.4(0.1)$ & $1.1(0.2)$ & $23.33(09)$ & $2.38(20)$ \\
\hline S88B & $5.0(0.3)$ & $15.6(0.9)$ & $21.60(03)$ & $2.81(07)$ & $1.7(0.3)$ & $4.7(0.7)$ & $21.88(05)$ & $2.23(12)$ & $0.5(.06)$ & $1.3(0.1)$ & 21.53(05) & $2.36(13)$ \\
\hline K3-50 & $1.3(0.2)$ & $10.6(0.8)$ & $-24.75(09)$ & $7.24(25)$ & $\ldots$ & $\ldots$ & $\ldots$ & $\ldots$ & $0.1(.06)$ & $0.9(0.2)$ & $-25.09(31)$ & 7.61(70) \\
\hline ON 1 & $2.5(0.1)$ & $15.2(0.5)$ & $11.56(03)$ & $5.44(08)$ & $3.1(0.6)$ & $12.7(1.9)$ & $11.44(08)$ & $4.18(18)$ & $0.5(.13)$ & $2.1(0.5)$ & $11.70(13)$ & $4.21(28)$ \\
\hline $\mathrm{ON} 2 \mathrm{~S}$ & $4.1(0.3)$ & $24.2(1.2)$ & $-1.13(05)$ & $5.22(12)$ & $4.5(0.7)$ & $22.1(2.3)$ & $-1.58(06)$ & $4.54(15)$ & $0.5(.07)$ & $2.0(0.2)$ & $-1.29(06)$ & $3.65(14)$ \\
\hline $\mathrm{ON} 2 \mathrm{~N}$ & $\ldots$ & $\ldots$ & $\ldots$ & $\ldots$ & $3.8(0.7)$ & $21.4(2.8)$ & $0.28(09)$ & $5.23(20)$ & $0.8(0.1)$ & $3.2(0.3)$ & $0.34(05)$ & $3.72(11)$ \\
\hline S106 & $2.8(0.1)$ & $10.2(0.4)$ & $-1.28(02)$ & $2.92(07)$ & $1.8(0.4)$ & 9.4(1.6) & $-2.37(11)$ & $4.91(24)$ & & $\ldots$ & $\ldots$ & $\ldots$ \\
\hline W75N & $6.5(0.2)$ & $29.0(0.7)$ & $9.41(02)$ & $4.14(04)$ & $11.3(0.5)$ & $65.4(2.1)$ & $9.48(02)$ & $5.02(06)$ & $1.5(0.1)$ & $7.0(0.4)$ & $9.64(04)$ & $4.22(11)$ \\
\hline DR21 S & $\ldots$ & $\ldots$ & $\ldots$ & $\ldots$ & $\ldots$ & $\ldots$ & $\ldots$ & $\ldots$ & $1.4(0.2)$ & $8.1(0.6)$ & $-2.29(06)$ & 4.94(19) \\
\hline W75(OH) & $6.7(0.2)$ & $37.4(0.7)$ & $-3.57(02)$ & $5.31(04)$ & $15.6(0.4)$ & $92.3(1.5)$ & $-3.64(01)$ & $5.47(03)$ & $1.3(0.2)$ & $7.4(0.7)$ & $-2.73(08)$ & $5.45(17)$ \\
\hline BFS 11-B & $\ldots$ & $\ldots$ & $\ldots$ & $\ldots$ & $1.5(0.4)$ & $5.8(1.4)$ & $-9.79(12)$ & $3.73(31)$ & & & $\ldots$ & \\
\hline CepA & $5.1(0.3)$ & $25.5(1.1)$ & $-11.05(03)$ & $4.58(08)$ & $\ldots$ & $\ldots$ & $\ldots$ & $\ldots$ & $0.8(.15)$ & $3.0(0.4)$ & $-10.39(09)$ & $3.68(19)$ \\
\hline S158 & $7.8(0.3)$ & $45.7(1.3)$ & $-56.56(03)$ & $5.25(06)$ & $\ldots$ & $\ldots$ & $\ldots$ & $\ldots$ & $1.2(0.1)$ & $5.2(0.4)$ & $-55.74(05)$ & $4.30(13)$ \\
\hline S158A & 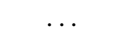 & $\ldots$ & & $\ldots$ & $\ldots$ & $\ldots$ & $\ldots$ & $\ldots$ & $1.1(0.3)$ & $4.1(0.9)$ & $-57.21(11)$ & $3.64(27)$ \\
\hline S157 & $2.9(0.2)$ & $11.5(0.8)$ & $-43.57(04)$ & $3.50(10)$ & $1.9(0.4)$ & 7.1(1.1) & $-44.70(07)$ & $3.53(18)$ & $\ldots$ & $\ldots$ & $\ldots$ & $\ldots$ \\
\hline IRAS18586+010 & $0.9(.15)$ & $3.8(0.5)$ & $38.42(10)$ & $3.60(29)$ & & $\ldots$ & & & & $\ldots$ & $\ldots$ & \\
\hline IRAS19094+0944 & $0.8(.14)$ & $2.1(0.5)$ & $65.47(08)$ & $2.72(20)$ & & $\ldots$ & $\ldots$ & $\ldots$ & $\ldots$ & $\ldots$ & $\ldots$ & \\
\hline IRAS20050+2720 & $1.5(0.3)$ & $5.5(0.8)$ & $6.48(09)$ & $3.19(22)$ & $\cdots$ & $\ldots$ & $\ldots$ & $\ldots$ & $\ldots$ & $\ldots$ & $\ldots$ & $\ldots$ \\
\hline
\end{tabular}


Table 3

(Continued)

\begin{tabular}{|c|c|c|c|c|c|c|c|c|c|c|c|c|}
\hline \multirow[t]{2}{*}{ Source } & \multicolumn{4}{|c|}{$\mathrm{CS} 2 \rightarrow 1$} & \multicolumn{4}{|c|}{$\mathrm{CS} 7 \rightarrow 6$} & \multicolumn{4}{|c|}{$\mathrm{C}^{34} \mathrm{~S} 5 \rightarrow 4$} \\
\hline & $\begin{array}{l}T_{R}^{*} \\
(\mathrm{~K})\end{array}$ & $\underset{\left(\mathrm{K} \mathrm{km} \mathrm{s}^{-1}\right)}{\int T_{R}^{*} \mathrm{~d} v}$ & $\begin{array}{c}V_{\mathrm{LSR}} \\
\left(\mathrm{km} \mathrm{s}^{-1}\right)\end{array}$ & $\begin{array}{c}\text { FWHM } \\
\left(\mathrm{km} \mathrm{s}^{-1}\right)\end{array}$ & $\begin{array}{l}T_{R}^{*} \\
(\mathrm{~K})\end{array}$ & $\underset{\left(\mathrm{K} \mathrm{km} \mathrm{s} \mathrm{s}^{-1}\right)}{\int T_{R}^{*} \mathrm{~d} v}$ & $\begin{array}{c}V_{\mathrm{LSR}} \\
\left(\mathrm{km} \mathrm{s}^{-1}\right)\end{array}$ & $\begin{array}{c}\text { FWHM } \\
\left(\mathrm{km} \mathrm{s}^{-1}\right)\end{array}$ & $\begin{array}{l}T_{R}^{*} \\
(\mathrm{~K})\end{array}$ & $\underset{\left(\mathrm{K} \mathrm{km} \mathrm{s}^{-1}\right)}{\int T_{R}^{*} \mathrm{~d} v}$ & $\begin{array}{c}V_{\mathrm{LSR}} \\
\left(\mathrm{km} \mathrm{s}^{-1}\right)\end{array}$ & $\begin{array}{c}\text { FWHM } \\
\left(\mathrm{km} \mathrm{s}^{-1}\right)\end{array}$ \\
\hline IRAS20106+3545 & $1.5(0.1)$ & $3.4(0.3)$ & 7.97(03) & $2.04(08)$ & $\ldots$ & $\ldots$ & $\ldots$ & $\ldots$ & $\ldots$ & $\ldots$ & $\ldots$ & $\ldots$ \\
\hline IRAS20126+4104 & $2.6(0.2)$ & $9.3(0.5)$ & $-3.52(03)$ & $3.09(07)$ & $\ldots$ & $\ldots$ & $\ldots$ & $\ldots$ & $\ldots$ & $\ldots$ & $\ldots$ & $\ldots$ \\
\hline IRAS20188+3928 & $3.6(0.2)$ & $16.3(0.9)$ & $2.05(03)$ & $3.70(09)$ & $\ldots$ & $\ldots$ & $\ldots$ & $\ldots$ & $0.2(.12)$ & $0.7(.35)$ & $1.74(33)$ & $3.75(92)$ \\
\hline IRAS20216+4107 & $1.1(0.2)$ & $2.7(0.5)$ & $-1.70(06)$ & $2.12(17)$ & $\ldots$ & $\ldots$ & $\ldots$ & $\ldots$ & $\ldots$ & $\ldots$ & $\ldots$ & $\ldots$ \\
\hline IRAS20220+3728 & $2.0(0.2)$ & $8.4(0.6)$ & $-2.16(05)$ & $3.61(13)$ & $\ldots$ & $\ldots$ & $\ldots$ & $\ldots$ & $\ldots$ & $\ldots$ & $\ldots$ & $\ldots$ \\
\hline IRAS20333+4102 & $3.5(0.1)$ & $6.9(0.4)$ & $8.55(01)$ & $1.84(03)$ & $\ldots$ & $\ldots$ & $\ldots$ & $\ldots$ & $\ldots$ & $\ldots$ & $\ldots$ & $\ldots$ \\
\hline IRAS21391+5802 & $3.9(0.2)$ & $13.1(0.5)$ & 0.71(02) & $2.99(05)$ & $\ldots$ & $\ldots$ & $\ldots$ & $\ldots$ & $\ldots$ & $\ldots$ & $\ldots$ & $\ldots$ \\
\hline IRAS22172+5549 & $1.4(0.2)$ & $4.0(0.6)$ & $-43.36(07)$ & $2.61(17)$ & $\ldots$ & $\ldots$ & $\ldots$ & $\ldots$ & $\ldots$ & $\ldots$ & $\ldots$ & $\ldots$ \\
\hline IRAS22198+6336 & $2.1(0.1)$ & $5.1(0.4)$ & $-11.09(02)$ & $2.05(06)$ & $\ldots$ & $\ldots$ & $\ldots$ & $\ldots$ & $\ldots$ & $\ldots$ & $\ldots$ & $\ldots$ \\
\hline IRAS23011+6126 & $1.4(0.2)$ & $3.8(0.5)$ & $-11.22(06)$ & $2.07(18)$ & $\ldots$ & $\ldots$ & $\ldots$ & $\ldots$ & $\ldots$ & $\ldots$ & $\ldots$ & $\ldots$ \\
\hline IRAS23385+6053 & $1.8(0.2)$ & 7.1(0.7) & $-49.47(07)$ & $3.90(14)$ & $\ldots$ & $\ldots$ & $\ldots$ & $\ldots$ & $\ldots$ & $\ldots$ & $\ldots$ & $\ldots$ \\
\hline
\end{tabular}

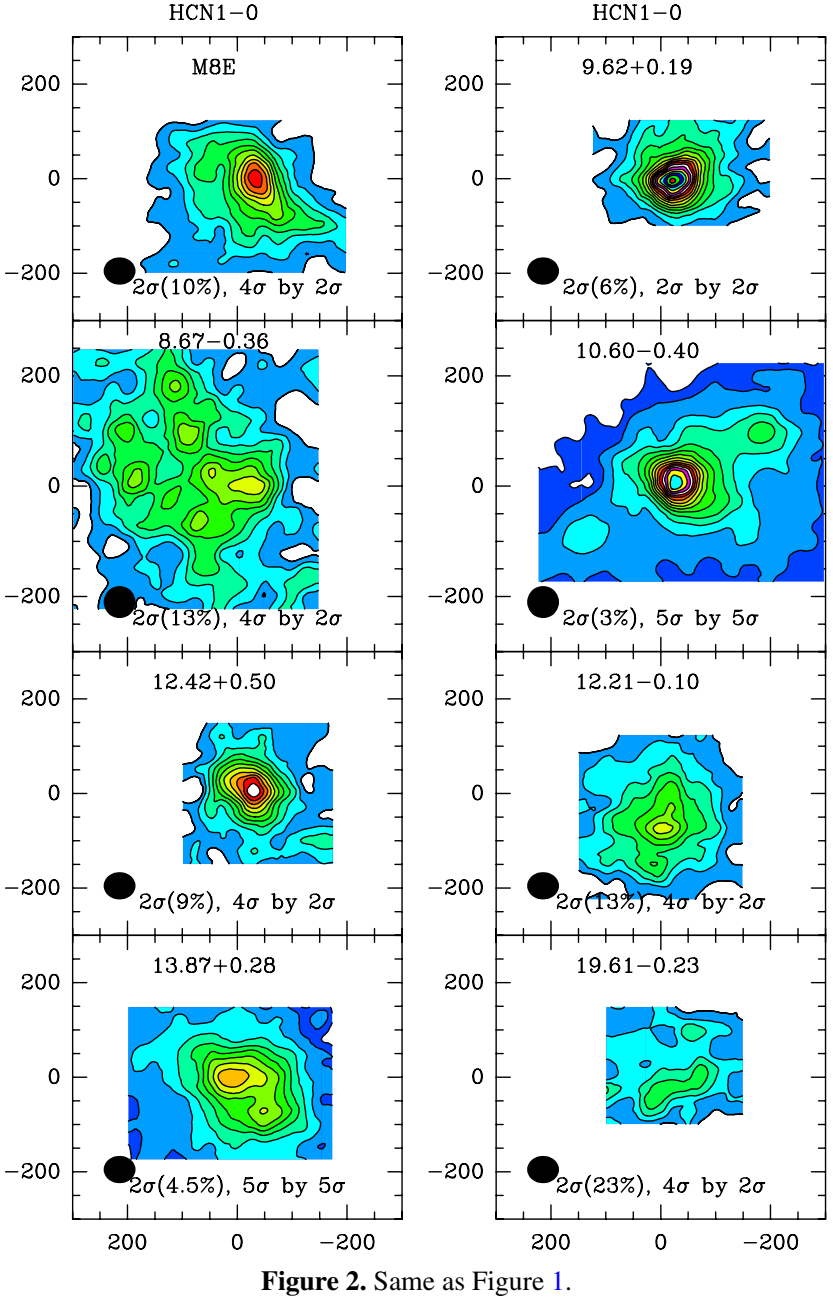

(A color version of this figure is available in the online journal.) out of 55). The majority of HCN 3-2 and CS 7-6 maps show a single, well-peaked distribution of intensity, similar to earlier maps of the CS 5-4 transition or the $350 \mu \mathrm{m}$ dust continuum emission (Shirley at al. 2003; Mueller et al. 2002).

Considering only sources with maps of multiple lines of the same species, there are 37 dense clumps mapped in both HCN $1-0$ and $\mathrm{HCN} 3-2$; six clumps that are single clumps in HCN 1-0 maps break into multiple clumps in HCN 3-2. Among the 38 clumps mapped by both CS 2-1 and CS 5-4, four single clumps
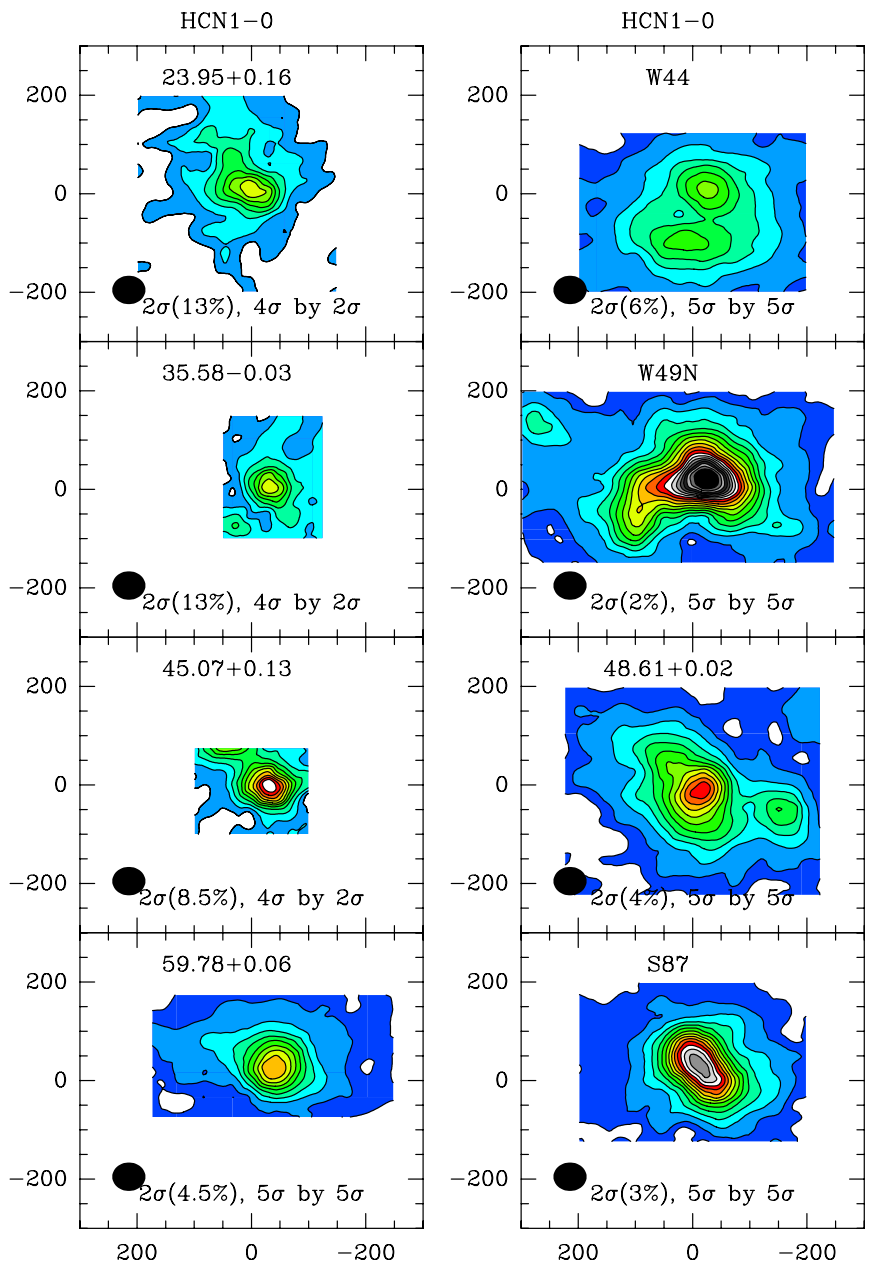

Figure 3. Same as Figure 1.

(A color version of this figure is available in the online journal.)

in CS 2-1 maps show multiple components in CS 5-4 maps. This effect may be due to the higher resolution of the HCN 3-2 and CS 5-4 observations. For the four clumps that have been resolved by CS 5-4 but not CS 2-1 maps, they are either not observed by CS 7-6, or the other components are beyond the CS 7-6 mapping area. Consequently, we do not know if they are also separate peaks in the CS 7-6 maps.

The overall impression is that low excitation lines may show secondary peaks that are not dense enough to show up in 
Table 4

Observational Results of $\mathrm{HCN} 3-2$ and $\mathrm{H}^{13} \mathrm{CN} 3-2$ Transitions

\begin{tabular}{|c|c|c|c|c|c|c|c|c|c|c|}
\hline \multirow[t]{2}{*}{ Source } & \multicolumn{4}{|c|}{$\mathrm{H}^{13} \mathrm{CN} 3 \rightarrow 2$} & \multicolumn{4}{|c|}{$\mathrm{HCN} 3 \rightarrow 2$} & \multirow{2}{*}{$\begin{array}{l}\text { Line }^{a} \\
\text { Profile }\end{array}$} & \multirow[t]{2}{*}{$\delta v$} \\
\hline & $\begin{array}{c}T_{R}^{*} \\
(\mathrm{~K})\end{array}$ & $\begin{array}{c}\int T_{R}^{*} \mathrm{~d} v \\
\left(\mathrm{~K} \mathrm{~km} \mathrm{~s}^{-1}\right)\end{array}$ & $\begin{array}{c}V_{\mathrm{LSR}} \\
\left(\mathrm{km} \mathrm{s}^{-1}\right)\end{array}$ & $\begin{array}{l}\text { FWHM } \\
\left(\mathrm{km} \mathrm{s}^{-1}\right)\end{array}$ & $\begin{array}{l}T_{R}^{*} \\
(\mathrm{~K}) \\
\end{array}$ & $\begin{array}{c}V_{\text {peak }} \\
\left(\mathrm{km} \mathrm{s}^{-1}\right)\end{array}$ & $\underset{\left(\mathrm{K} \mathrm{km} \mathrm{s}^{-1}\right)}{\int T_{R}^{*} \mathrm{~d} \nu}$ & $\begin{array}{c}V_{\mathrm{LSR}} \\
\left(\mathrm{km} \mathrm{s}^{-1}\right)\end{array}$ & & \\
\hline G121.30+0.66 & $0.5(.11)$ & $1.8(0.3)$ & $-17.77(10)$ & $3.06(25)$ & $4.1(.30)$ & $-18.42(22)$ & $22.6(1.1)$ & $-17.00(22)$ & $\mathrm{db}$ & $-0.37(08)$ \\
\hline G123.07-6.31 & $0.5(.11)$ & $2.8(0.3)$ & $-30.79(13)$ & $4.87(32)$ & $2.5(.23)$ & $-32.75(22)$ & $22.5(1.0)$ & $-28.90(22)$ & $\mathrm{db}$ & $-0.46(12)$ \\
\hline $\mathrm{W} 3(2)$ & $0.7(.12)$ & $3.3(0.4)$ & $-38.73(10)$ & $4.48(25)$ & $5.9(.29)$ & $-36.97(32)$ & $32.8(1.4)$ & $-39.77(32)$ & $\mathrm{dr}$ & $0.39(04)$ \\
\hline $\mathrm{W} 3(\mathrm{OH})$ & $1.8(.13)$ & $10.1(0.5)$ & $-47.16(05)$ & $5.30(12)$ & $6.6(.55)$ & $-49.23(22)$ & $51.3(2.3)$ & $-46.15(22)$ & $\mathrm{db}$ & $-0.32(03)$ \\
\hline $\mathrm{G} 135.28+2.80$ & $\ldots$ & $\ldots$ & $\ldots$ & $\ldots$ & $0.7(.21)$ & $-42.18(16)$ & $3.3(0.7)$ & $-42.18(19)$ & & \\
\hline S231 & $0.8(.10)$ & $3.3(0.4)$ & $-16.41(06)$ & $3.74(17)$ & $3.5(.23)$ & $-14.12(22)$ & $20.2(1.0)$ & $\cdots$ & rbs & $0.67(07)$ \\
\hline $\mathrm{S} 235$ & $1.1(.11)$ & $3.4(0.3)$ & $-17.44(05)$ & $2.71(13)$ & $5.0(.31)$ & $-17.36(11)$ & 29.1(1.0) & $-17.36(03)$ & & $-0.18(06)$ \\
\hline S241 & $\ldots$ & $\ldots$ & $\ldots$ & $\ldots$ & $1.1(.21)$ & $-8.58(22)$ & $5.4(0.7)$ & $-8.58(11)$ & & \\
\hline MonR2 & $\ldots$ & $\ldots$ & $\ldots$ & $\ldots$ & $7.8(.23)$ & $10.19(16)$ & $39.0(0.9)$ & $10.31(02)$ & & \\
\hline $\mathrm{S} 252 \mathrm{~A}$ & $0.6(.09)$ & $1.7(0.3)$ & $9.13(07)$ & $2.61(16)$ & $3.7(.32)$ & $10.04(22)$ & $20.5(1.2)$ & $9.65(06)$ & rbs & $0.35(05)$ \\
\hline S255 & $\ldots$ & $\ldots$ & $\ldots$ & $\ldots$ & $7.9(.68)$ & $7.98(22)$ & $48.1(2.5)$ & $7.65(05)$ & & $0.21(05)$ \\
\hline RCW142 & $2.7(.27)$ & $21.6(1.0)$ & $16.16(09)$ & $7.15(23)$ & $17.8(.45)$ & $14.36(11)$ & $164.9(2.6)$ & $20.77(21)$ & $\mathrm{db}$ & $-0.53(02)$ \\
\hline W28A2(1) & $5.1(.21)$ & $47.8(1.3)$ & $9.23(03)$ & $6.93(09)$ & 13.1(.35) & $11.18(22)$ & $177.3(2.3)$ & $11.40(03)$ & ws & $0.24(02)$ \\
\hline G9.62+0.10 & $1.2(.19)$ & $10.9(0.8)$ & 4.77(10) & $8.17(26)$ & $6.2(.60)$ & $7.38(22)$ & $52.0(2.9)$ & 2.67(42) & $\mathrm{dr}$ & $0.46(02)$ \\
\hline G8.67-0.36 & $1.4(.33)$ & $6.9(1.0)$ & $34.32(13)$ & $4.40(30)$ & $2.7(.16)$ & $31.91(16)$ & $22.4(0.8)$ & $36.19(50)$ & $\mathrm{db}$ & $-0.58(04)$ \\
\hline W31 (1) & $1.1(.11)$ & $7.9(2.0)$ & $64.85(08)$ & $6.69(19)$ & $6.9(.22)$ & $62.71(11)$ & $68.2(1.1)$ & $68.70(21)$ & $\mathrm{db}$ & $-0.42(02)$ \\
\hline G10.60-0.40 & $3.6(.21)$ & $30.5(0.9)$ & $-4.16(06)$ & $7.74(14)$ & $11.8(.62)$ & $-6.50(11)$ & $132.2(3.4)$ & $-0.43(10)$ & $\mathrm{db}$ & $-0.54(02)$ \\
\hline $\mathrm{G} 12.42+0.50$ & $0.9(.26)$ & $1.7(0.6)$ & $17.36(12)$ & $1.79(29)$ & $4.2(.25)$ & $16.06(11)$ & 24.1(0.9) & $17.49(09)$ & $\mathrm{db}$ & $-0.73(15)$ \\
\hline G12.89+0.49 & $0.8(.19)$ & $5.4(0.8)$ & $33.13(18)$ & $6.42(48)$ & $2.6(.15)$ & $\ldots$ & $21.1(0.7)$ & $33.49(30)$ & $\mathrm{d}$ & \\
\hline W33cont & $2.1(.34)$ & $11.6(1.4)$ & $35.43(11)$ & $5.74(24)$ & $10.0(.38)$ & $39.53(22)$ & $94.3(2.1)$ & $33.75(20)$ & $\mathrm{dr}$ & $0.83(02)$ \\
\hline W33A & $0.7(.23)$ & $4.2(0.8)$ & $37.69(22)$ & $5.40(58)$ & $1.8(.21)$ & $40.49(22)$ & $25.8(1.2)$ & $38.14(11)$ & rbs & $0.81(07)$ \\
\hline G14.33-0.64 & $1.8(.30)$ & $8.8(1.2)$ & $22.25(10)$ & $4.59(25)$ & $7.1(.25)$ & $25.47(11)$ & $69.1(1.5)$ & $21.65(10)$ & $\mathrm{dr}$ & $1.20(05)$ \\
\hline G19.61-0.23 & $0.4(.11)$ & $6.3(0.7)$ & $42.57(34)$ & $15.50(85)$ & $3.1(.32)$ & $40.40(22)$ & $45.2(1.9)$ & $40.66(14)$ & brs & $-0.14(02)$ \\
\hline G20.08-0.13 & $\ldots$ & $\ldots$ & $\ldots$ & $\ldots$ & $2.1(.30)$ & $38.46(32)$ & $22.3(1.5)$ & $39.85(16)$ & brs & \\
\hline $\mathrm{G} 23.95+0.16$ & $0.4(.09)$ & $1.3(0.3)$ & $80.32(10)$ & $3.24(35)$ & $1.9(.28)$ & $78.57(22)$ & $14.5(1.1)$ & $80.52(22)$ & $\mathrm{db}$ & $-0.71(09)$ \\
\hline W42 & $0.9(.32)$ & $8.4(1.4)$ & $110.49(28)$ & $8.55(58)$ & $3.9(.35)$ & $114.49(22)$ & $28.1(1.8)$ & $108.59(22)$ & $\mathrm{dr}$ & $0.47(05)$ \\
\hline W43S & $1.3(.16)$ & $7.1(0.7)$ & 98.11(06) & $5.25(16)$ & $6.3(.32)$ & $96.61(22)$ & $52.0(1.4)$ & $96.85(03)$ & brs & $-0.35(03)$ \\
\hline G31.41+0.31 & $1.2(.30)$ & $7.7(1.2)$ & $96.23(16)$ & $5.41(46)$ & $3.2(.28)$ & $93.25(16)$ & $21.5(1.5)$ & $98.67(32)$ & $\mathrm{db}$ & $-0.63(03)$ \\
\hline G31.44-0.26 & $\ldots$ & $\ldots$ & $\ldots$ & $\ldots$ & $3.1(.19)$ & $87.87(16)$ & $24.8(0.8)$ & $87.27(05)$ & & $0.21(06)$ \\
\hline W44 & $3.8(.14)$ & $24.4(0.6)$ & $56.82(04)$ & $5.64(10)$ & $11.5(.32)$ & $54.79(16)$ & $84.9(1.8)$ & $61.17(22)$ & $\mathrm{db}$ & $-0.86(04)$ \\
\hline S76E & $\ldots$ & $\ldots$ & $\ldots$ & $\ldots$ & $7.8(.23)$ & $33.63(16)$ & $53.6(1.0)$ & $32.99(02)$ & rbs & \\
\hline G35.58-0.03 & $0.3(.06)$ & $1.8(0.2)$ & $53.68(13)$ & $6.13(34)$ & $2.8(.34)$ & $52.91(22)$ & $30.0(1.7)$ & $52.91(09)$ & & $-0.18(06)$ \\
\hline G35.20-0.74 & $0.7(.20)$ & $6.5(0.8)$ & $34.01(24)$ & $8.26(56)$ & $7.9(.26)$ & $37.34(16)$ & $54.3(1.2)$ & $33.93(32)$ & $\mathrm{dr}$ & $0.77(06)$ \\
\hline W49N & $1.2(.39)$ & $\ldots$ & $\ldots$ & $\ldots$ & $8.7(.33)$ & $3.02(22)$ & $142.4(2.0)$ & $12.44(22)$ & two & \\
\hline W49S & $\ldots$ & $\ldots$ & $\ldots$ & $\ldots$ & $2.8(.16)$ & $15.32(16)$ & $37.3(0.9)$ & $15.32(06)$ & & $0.07(04)$ \\
\hline $\mathrm{OH} 43.80-0.13$ & $0.5(.21)$ & $4.8(0.9)$ & $44.17(31)$ & $8.47(77)$ & $2.2(.25)$ & $46.80(32)$ & $23.7(1.2)$ & $42.96(32)$ & $\mathrm{dr}$ & $0.31(05)$ \\
\hline G45.07+0.13 & $0.6(.16)$ & $4.3(0.7)$ & $59.13(15)$ & $6.49(35)$ & $4.3(.27)$ & $59.52(22)$ & $41.8(1.5)$ & $59.52(04)$ & rw & $0.04(02)$ \\
\hline $\mathrm{G} 48.61+0.02$ & $0.2(.09)$ & $1.4(0.3)$ & $18.04(22)$ & $5.35(67)$ & $2.3(.34)$ & $18.09(11)$ & $18.7(1.4)$ & $18.09(09)$ & & $-0.02(07)$ \\
\hline W51W & $\ldots$ & $\ldots$ & $\ldots$ & $\ldots$ & $2.5(.32)$ & $52.18(32)$ & $18.5(1.2)$ & $51.44(09)$ & $\mathrm{sr}$ & $0.35(06)$ \\
\hline W51M & $3.1(.47)$ & $36.3(2.3)$ & $56.60(15)$ & $11.04(37)$ & $14.2(.44)$ & $51.74(32)$ & $153.9(2.3)$ & $55.65(99)$ & brs & $-0.59(02)$ \\
\hline G59.78+0.06 & $0.2(.11)$ & $0.4(0.2)$ & $22.67(18)$ & $1.96(36)$ & $2.5(.27)$ & $21.52(22)$ & $16.0(1.0)$ & 21.79(06) & $\mathrm{sb}$ & $-0.55(10)$ \\
\hline S87 & $\ldots$ & $\ldots$ & $\ldots$ & $\ldots$ & $4.6(.28)$ & $22.76(22)$ & $25.9(1.0)$ & $22.67(03)$ & brs & $-0.24(06)$ \\
\hline S88B & $0.4(.07)$ & $1.3(0.2)$ & $21.85(08)$ & $3.11(18)$ & $2.8(.25)$ & $22.06(22)$ & $15.8(0.9)$ & $22.06(05)$ & & $0.22(05)$ \\
\hline K3-50 & $\ldots$ & $\ldots$ & $\ldots$ & $\ldots$ & $4.1(.26)$ & $-22.91(22)$ & $40.3(1.3)$ & $-23.78(05)$ & $\mathrm{sr}$ & $0.29(05)$ \\
\hline ON 1 & $0.8(.23)$ & $3.9(0.8)$ & $11.66(17)$ & $4.74(42)$ & $3.8(.40)$ & $13.87(22)$ & $27.2(1.8)$ & $10.72(22)$ & $\mathrm{dr}$ & $0.52(05)$ \\
\hline $\mathrm{ON} 2 \mathrm{~S}$ & $0.7(.16)$ & $3.1(0.6)$ & $-1.50(11)$ & $4.31(30)$ & $8.6(.33)$ & $-0.07(22)$ & $68.0(1.6)$ & $-1.59(03)$ & rbs & $0.33(04)$ \\
\hline $\mathrm{ON} 2 \mathrm{~N}$ & $\ldots$ & $\ldots$ & $\ldots$ & $\ldots$ & $4.2(.40)$ & $2.58(22)$ & $46.1(2.0)$ & $0.51(09)$ & rbs & $0.60(04)$ \\
\hline S106 & $\ldots$ & $\ldots$ & $\ldots$ & $\ldots$ & $3.0(.35)$ & $-2.38(22)$ & $20.2(1.4)$ & $-3.00(07)$ & rbs & \\
\hline W75N & $1.9(.22)$ & $11.1(1.0)$ & $9.67(06)$ & $5.12(16)$ & $9.5(.27)$ & $11.74(22)$ & $92.3(1.7)$ & $9.46(11)$ & $\mathrm{dr}$ & $0.50(03)$ \\
\hline DR21 S & 1.7(.19) & $15.2(0.9)$ & $-2.16(10)$ & $8.39(26)$ & $15.8(.36)$ & $-6.02(22)$ & $217.7(2.3)$ & $-2.00(11)$ & $\mathrm{db}$ & $-0.76(04)$ \\
\hline W75(OH) & $1.8(.26)$ & $10.3(1.0)$ & $-3.35(13)$ & $5.26(28)$ & $11.1(.43)$ & $-6.27(22)$ & $80.7(2.2)$ & $-2.24(22)$ & $\mathrm{db}$ & $-0.65(03)$ \\
\hline G97.53+3.19 & $\ldots$ & $\ldots$ & $\ldots$ & $\ldots$ & $1.0(.32)$ & $-71.88(22)$ & $8.3(1.5)$ & $-71.88(27)$ & & \\
\hline CepA & $\ldots$ & $\ldots$ & $\ldots$ & $\ldots$ & $7.9(.37)$ & $-12.33(22)$ & $71.5(2.0)$ & $-10.41(22)$ & $\mathrm{db}$ & $-0.53(05)$ \\
\hline S158 & $0.9(.13)$ & $5.7(0.5)$ & $-56.29(11)$ & $6.09(26)$ & $5.5(.32)$ & $-59.05(22)$ & $52.0(1.8)$ & $-55.28(22)$ & $\mathrm{db}$ & $-0.77(04)$ \\
\hline S158A & & & & & $7.0(.82)$ & $-58.43(22)$ & $37.2(3.7)$ & $-58.13(10)$ & brs & $-0.34(05)$ \\
\hline NGC7538-IRS9 & $\ldots$ & $\ldots$ & $\ldots$ & $\ldots$ & $3.0(.33)$ & $-54.75(22)$ & $18.7(1.3)$ & $\cdots$ & rbs & \\
\hline S157 & $\ldots$ & $\ldots$ & $\ldots$ & $\ldots$ & $3.0(.82)$ & $-45.03(22)$ & $17.8(3.0)$ & $-44.53(15)$ & & $-0.36(06)$ \\
\hline IRAS $20050+2720$ & $\ldots$ & $\ldots$ & $\ldots$ & $\ldots$ & $2.3(.25)$ & $5.94(22)$ & $13.1(0.9)$ & 5.94(06) & & \\
\hline IRAS20216+4107 & $\ldots$ & $\ldots$ & $\ldots$ & $\ldots$ & $2.0(.28)$ & $-1.31(11)$ & $7.9(0.9)$ & $-1.31(06)$ & & \\
\hline IRAS23011+6126 & $\ldots$ & $\ldots$ & $\ldots$ & $\ldots$ & $0.9(.29)$ & $-10.94(22)$ & $7.4(1.0)$ & $-10.94(21)$ & & \\
\hline
\end{tabular}

Note.

${ }^{a}$ Code of line profiles: db, double peak with blue strong; dr, double peaks with red strong; d: double peaks with neither one obviously stronger; flat, the line has a flat top; rbs, blue shoulder with peak skewed to red; brs, red shoulder with peak skewed to blue; ws: wings on both sides; rw, red wing; sr, peak skews to red; br, peak skews to blue; two, the line has two known velocity components. 
Table 5

Observational Results for HCN 1-0 Transition

\begin{tabular}{|c|c|c|c|c|c|c|c|}
\hline Source & $\begin{array}{c}T_{R}^{*}(\mathrm{~K}) \\
(F=1-0)\end{array}$ & $\begin{array}{c}T_{R}^{*}(\mathrm{~K}) \\
(F=2-1) \\
\end{array}$ & $\begin{array}{c}T_{R}^{*}(\mathrm{~K}) \\
(F=1-1))\end{array}$ & $\begin{array}{c}\int T_{R}^{*} \mathrm{~d} \nu\left(\mathrm{K} \mathrm{km} \mathrm{s}^{-1}\right) \\
(\mathrm{All} \mathrm{hpf} F \mathrm{~s})\end{array}$ & $\begin{array}{c}V_{\mathrm{LSR}}\left(\mathrm{km} \mathrm{s}^{-1}\right) \\
(F=2-1)\end{array}$ & $\begin{array}{c}\text { FWHM }\left(\mathrm{km} \mathrm{s}^{-1}\right. \\
(F=2-1)\end{array}$ & $\begin{array}{l}\text { Line }^{\mathrm{a}} \\
\text { Profile }\end{array}$ \\
\hline G121.30+0.66 & $0.9(0.2)$ & $4.0(0.2)$ & $\ldots$ & $24.9(1.2)$ & $-18.31(33)$ & $\ldots$ & $a b$ \\
\hline G123.07-6.31 & $0.9(0.2)$ & $2.6(0.2)$ & $1.8(0.2)$ & $19.6(1.0)$ & $-31.13(04)$ & $3.63(05)$ & \\
\hline $\mathrm{W} 3(\mathrm{OH})$ & $1.9(0.1)$ & $\ldots$ & $\ldots$ & $41.8(0.7)$ & $\ldots$ & $\ldots$ & $\mathrm{ab}, \mathrm{bl}$ \\
\hline S231 & $0.7(0.2)$ & $2.3(0.2)$ & $1.3(0.2)$ & $20.1(1.2)$ & $-17.26(06)$ & $4.21(07)$ & bl \\
\hline $\mathrm{S} 235$ & $1.2(0.2)$ & $4.3(0.2)$ & $1.5(0.2)$ & $19.0(1.0)$ & $-17.17(02)$ & $2.37(03)$ & \\
\hline $\mathrm{S} 241$ & $0.3(0.1)$ & $1.5(0.1)$ & $0.7(0.1)$ & 7.1(0.5) & $-8.90(03)$ & $2.66(04)$ & \\
\hline S252A & $1.0(0.2)$ & $3.5(0.2)$ & $1.6(0.2)$ & $21.6(1.1)$ & $9.05(03)$ & $3.28(04)$ & \\
\hline RCW142 & $3.1(0.3)$ & $5.6(0.3)$ & $3.1(0.3)$ & 73.2(1.6) & $16.73(06)$ & $5.31(07)$ & bl \\
\hline W28A2(1) & $1.9(0.2)$ & $4.0(0.2)$ & $1.0(0.5)$ & 28.1(1.2) & $8.65(03)$ & $3.66(04)$ & \\
\hline M8E & $0.8(0.3)$ & $4.0(0.3)$ & $1.8(0.3)$ & $17.6(1.3)$ & $10.46(03)$ & $2.52(04)$ & \\
\hline G9.62+0.10 & $1.2(0.3)$ & $1.0(0.3)$ & $1.1(0.3)$ & $17.3(1.7)$ & $5.50(13)$ & $4.31(20)$ & \\
\hline G8.67-0.36 & $1.0(0.3)$ & $1.0(0.3)$ & $\ldots$ & $11.4(1.3)$ & $33.15(33)$ & $\ldots$ & \\
\hline G10.60-0.40 & $0.9(0.6)$ & $2.3(1.0)$ & $1.3(0.5)$ & $46.0(1.7)$ & $-3.45(01)$ & $\ldots$ & bf, bl \\
\hline $\mathrm{G} 12.42+0.50$ & $0.5(0.3)$ & $1.7(0.3)$ & $1.3(0.3)$ & $15.1(1.3)$ & 17.59(11) & $3.88(11)$ & bl \\
\hline $\mathrm{G} 12.21-0.10$ & $\ldots$ & $0.5(0.2)$ & $\ldots$ & $4.7(0.8)$ & $\ldots$ & $\ldots$ & bl \\
\hline $\mathrm{G} 13.87+0.28$ & $2.9(0.4)$ & $4.4(0.4)$ & $2.7(0.4)$ & $55.3(1.9)$ & $\ldots$ & $\ldots$ & bl \\
\hline G19.61-0.23 & $\ldots$ & $0.2(0.1)$ & $\ldots$ & $1.6(0.5)$ & $\ldots$ & $\ldots$ & \\
\hline $\mathrm{G} 23.95+0.16$ & $0.6(0.2)$ & $2.4(0.2)$ & $1.1(0.2)$ & $12.4(1.0)$ & $78.90(04)$ & $2.72(06)$ & \\
\hline W43S & $1.6(0.1)$ & $2.9(0.1)$ & $1.0(0.1)$ & $26.4(0.8)$ & $96.72(03)$ & $4.03(04)$ & \\
\hline W44 & $1.7(0.2)$ & $2.2(0.2)$ & $\ldots$ & $12.7(0.8)$ & $57.07(04)$ & $2.93(05)$ & \\
\hline G35.58-0.03 & $\ldots$ & $0.5(0.1)$ & $\ldots$ & $5.2(0.7)$ & $\ldots$ & $\ldots$ & bl \\
\hline W49N & $\ldots$ & $\ldots$ & $\ldots$ & $53.1(1.2)$ & $\ldots$ & $\ldots$ & bl \\
\hline G45.07+0.13 & $0.5(0.2)$ & $0.8(0.2)$ & $0.2(0.2)$ & $10.9(1.1)$ & $59.58(03)$ & $5.93(23)$ & bl \\
\hline G48.61+0.02 & $0.5(0.1)$ & $2.1(0.1)$ & $1.1(0.1)$ & $17.9(0.7)$ & $18.36(03)$ & $4.28(04)$ & bl \\
\hline W51M & $\ldots$ & $\ldots$ & $\ldots$ & $50.4(1.1)$ & $\ldots$ & $\ldots$ & bl \\
\hline G59.78+0.06 & $0.5(0.2)$ & $2.1(0.2)$ & $1.0(0.2)$ & $12.3(0.9)$ & $22.45(05)$ & $3.01(06)$ & \\
\hline S87 & $1.1(0.2)$ & $4.8(0.2)$ & $2.1(0.2)$ & $31.2(0.8)$ & $22.88(02)$ & $3.71(02)$ & bl2 \\
\hline S88B & $0.9(0.2)$ & $3.9(0.2)$ & $2.3(0.2)$ & $25.2(0.9)$ & $21.54(02)$ & $3.22(03)$ & bl2 \\
\hline K3-50 & $\ldots$ & $\ldots$ & $\ldots$ & $24.3(0.8)$ & $\ldots$ & $\ldots$ & bl \\
\hline ON 1 & $\ldots$ & $\ldots$ & $\ldots$ & $16.0(0.7)$ & $\ldots$ & $\ldots$ & bl \\
\hline ON 2S & $\ldots$ & $\ldots$ & $\ldots$ & $2.5(0.8)$ & $\ldots$ & $\ldots$ & $\mathrm{ab}, \mathrm{bl}$ \\
\hline S106 & $0.5(0.1)$ & $2.0(0.4)$ & $1.0(0.1)$ & $12.7(0.4)$ & $-1.58(02)$ & $3.22(03)$ & bl2 \\
\hline W75N & $0.8(0.1)$ & $1.4(0.1)$ & $1.0(0.1)$ & $19.3(0.5)$ & $9.75(07)$ & $5.54(08)$ & bl \\
\hline DR21 S & $1.2(0.2)$ & $3.4(0.2)$ & $1.4(0.2)$ & $19.4(1.1)$ & $-3.49(07)$ & $5.69(09)$ & $\mathrm{ab}, \mathrm{bl}$ \\
\hline W75(OH) & $\ldots$ & $\ldots$ & $\ldots$ & $21.7(1.0)$ & $\ldots$ & $\ldots$ & $\mathrm{ab}, \mathrm{bl}$ \\
\hline CepA & $1.0(0.2)$ & $\ldots$ & $\ldots$ & $30.6(0.9)$ & $\ldots$ & $\ldots$ & $\mathrm{bl}, \mathrm{bf}$ \\
\hline S158 & $3.2(0.3)$ & $5.3(0.3)$ & $1.9(0.3)$ & $57.9(1.5)$ & $-57.14(04)$ & $4.76(05)$ & bl \\
\hline S157 & $0.7(0.2)$ & $2.7(0.2)$ & $1.3(0.2)$ & $19.5(0.9)$ & $-43.58(04)$ & $3.79(05)$ & $\mathrm{b} 12$ \\
\hline IRAS18586+0106 & $0.3(0.2)$ & $0.7(0.2)$ & $0.2(0.2)$ & $4.1(0.7)$ & $38.34(10)$ & $3.10(15)$ & \\
\hline IRAS19094+0944 & $0.2(0.1)$ & $0.8(0.1)$ & $0.5(0.1)$ & $5.1(0.6)$ & $65.48(07)$ & $3.13(08)$ & \\
\hline IRAS20050+2720 & $0.3(0.2)$ & $0.5(0.2)$ & $0.3(0.2)$ & $5.7(1.1)$ & $6.06(27)$ & $4.72(25)$ & bl2 \\
\hline IRAS20106+3545 & $0.3(0.1)$ & $1.3(0.1)$ & $0.6(0.1)$ & $5.2(0.5)$ & $7.80(03)$ & $2.15(05)$ & \\
\hline IRAS20126+4104 & $0.9(0.1)$ & $2.9(0.1)$ & $1.8(0.1)$ & $19.6(0.6)$ & $-3.60(02)$ & $3.14(02)$ & bl2 \\
\hline IRAS20188+3928 & $0.7(0.2)$ & $1.9(0.5)$ & $1.3(0.4)$ & $19.8(0.8)$ & $2.49(06)$ & $4.42(07)$ & $\mathrm{ab}$ ? bl, bf \\
\hline IRAS20216+4107 & $0.2(0.1)$ & $1.3(0.1)$ & $0.5(0.1)$ & $5.4(0.6)$ & $-1.57(04)$ & $2.32(06)$ & \\
\hline IRAS20220+3728 & $0.4(0.2)$ & $1.8(0.2)$ & $0.3(0.2)$ & $8.9(0.8)$ & $-2.30(05)$ & $3.09(06)$ & \\
\hline IRAS20333+4102 & $0.9(0.1)$ & $4.4(0.1)$ & $1.8(0.1)$ & $16.9(0.6)$ & $8.60(01)$ & $2.21(02)$ & \\
\hline IRAS21391+5802 & $0.9(0.1)$ & $1.8(0.5)$ & $1.2(0.2)$ & $15.0(0.6)$ & $0.50(03)$ & $3.30(04)$ & $a b ?$ \\
\hline IRAS22172+5549 & $0.2(0.1)$ & $1.2(0.1)$ & $0.5(0.1)$ & $6.2(0.7)$ & $-43.51(06)$ & $3.05(07)$ & $\mathrm{bl} 2$ \\
\hline IRAS22198+6336 & $0.5(0.1)$ & $1.3(0.1)$ & $0.5(0.1)$ & $6.0(0.6)$ & $-11.14(04)$ & $2.35(05)$ & \\
\hline IRAS23011+6126 & $0.4(0.1)$ & $0.9(0.1)$ & $0.6(0.1)$ & $6.7(0.6)$ & $-11.76(07)$ & $3.25(09)$ & \\
\hline IRAS23385+6053 & $0.5(0.2)$ & $1.9(0.2)$ & $0.8(0.2)$ & $10.0(0.8)$ & $-49.95(04)$ & $2.91(05)$ & \\
\hline IRAS03282+3035 & $0.8(0.1)$ & $1.3(0.1)$ & $0.8(0.1)$ & $3.3(0.4)$ & 6.94(02) & $0.92(04)$ & \\
\hline L1251B & $0.7(0.1)$ & $1.5(0.3)$ & $0.9(0.2)$ & $5.0(0.3)$ & $-4.03(02)$ & $1.56(02)$ & $a b$ \\
\hline
\end{tabular}

Note.

a Code of line profiles: ab, absorption features on the line; bl, three components are blended; bl2: Only $F=2-1$ and $F=1-1$ are blended; bf, the line cannot be well fitted by three Gaussian components.

the lines requiring higher excitation, while maps with higher resolution may find multiple peaks within the low-resolution map. Because lines of higher excitation in our study have better spatial resolution, those multiple peaks may not be seen in the lower excitation tracers, just because of instrumental limitations. To check this possibility, we have smoothed the HCN 3-2 maps for four clumps with multiple peaks to the resolution of the $\mathrm{HCN}$ 1-0 data and compare them to the HCN 1-0 data (Figure 24). 

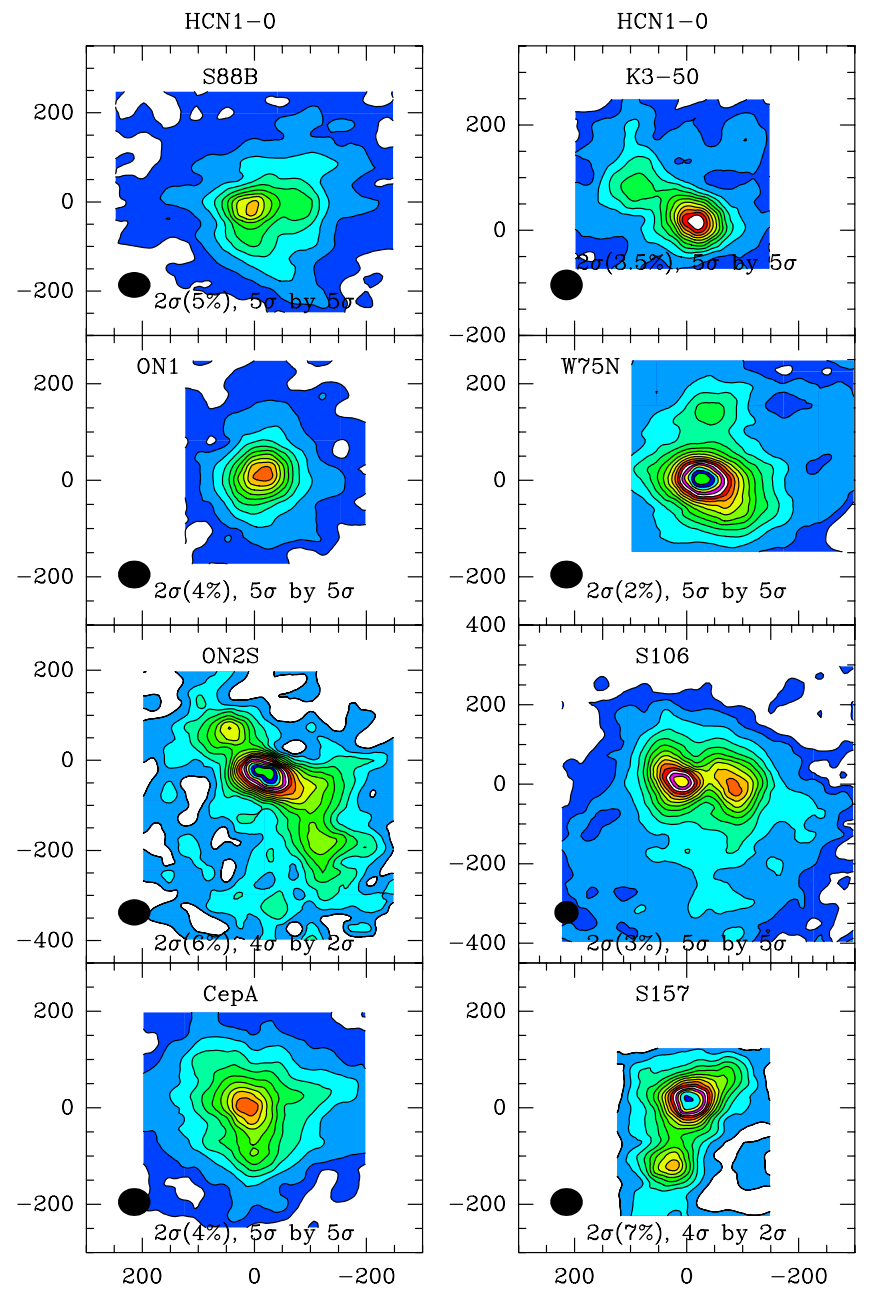

Figure 4. Same as Figure 1

(A color version of this figure is available in the online journal.)

The similarity of the smoothed HCN 3-2 maps to the HCN 1-0 maps supports the suggestion that $\mathrm{HCN} 1-0$ maps with higher resolution would also resolve multiple peaks.

These few clumps aside, the general appearance of most maps at the current resolution is not one of many cores within a clump, but of single well-peaked distributions. Maps with higher resolution that still retain sensitivity to extended structure are needed to study substructure in these clumps.

\subsection{Clump Sizes}

The size of the clump is characterized by the angular diameter or linear radius after beam deconvolution. $\theta_{\text {transition }}$ is the angular diameter of a circle that has the same area as the half-peak intensity contour:

$$
\theta_{\text {transition }}=2\left(\frac{A_{1 / 2}}{\pi}-\frac{\theta_{\text {beam }}^{2}}{4}\right)^{1 / 2},
$$

and $R_{\text {transition }}=\theta_{\text {transition }} D / 2$, where $A_{1 / 2}$ is the area within the contour of half-peak intensity, $\theta_{\text {beam }}$ is the FWHM beam size, and $D$ is the distance to the source. It is important to note that these sizes do not represent sharp boundaries. To show this fact, we chose G10.6-0.4, W3(OH), and S231 as examples, since these sources are round in the contour maps, thus better approximated by a one-dimensional radial profile. In Figure 25, the emission generally declines smoothly to the noise level,
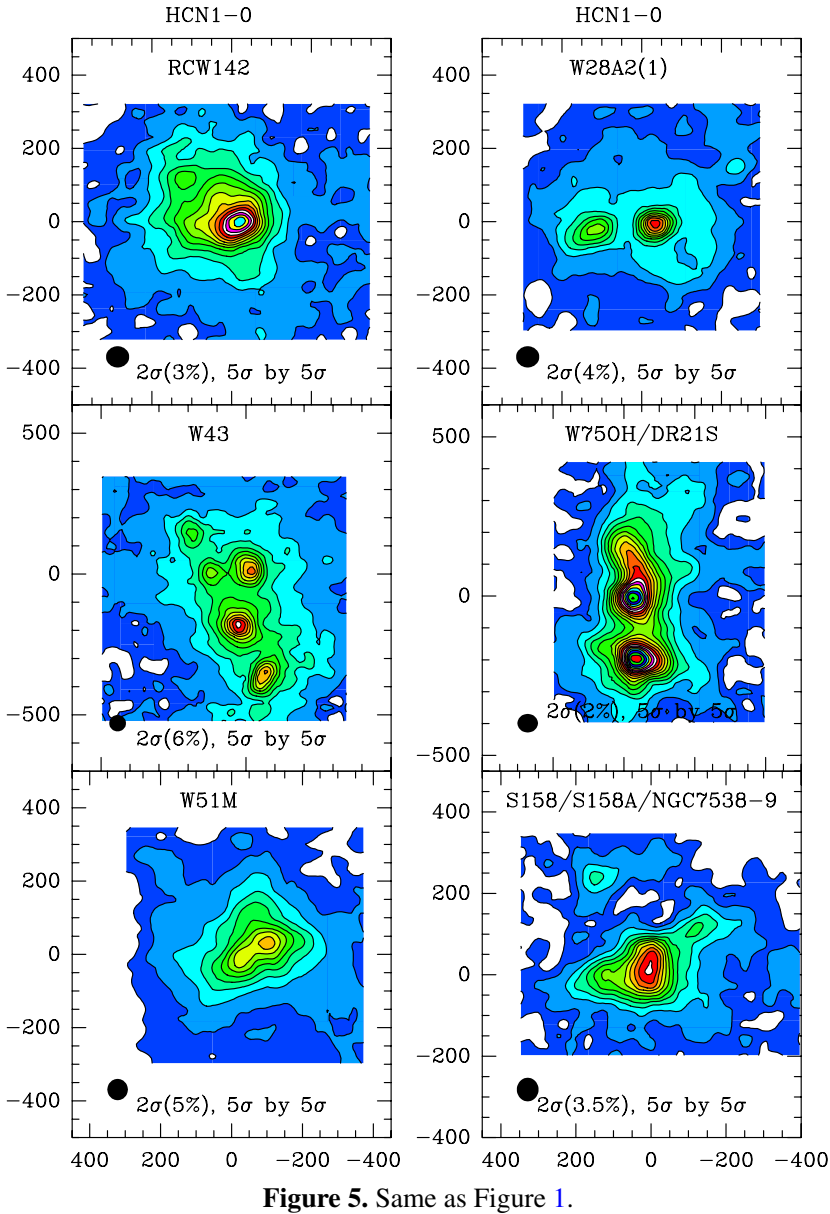

Figure 5. Same as Figure 1

(A color version of this figure is available in the online journal.)

when plotted against the angular source size, in units of the FWHM angular extent.

The calculated sizes of clumps in each transition are listed in Table 6, with the mean and median values given at the end of the table. The statistics on median and mean value include only the massive sources, but not those sources below the horizontal line in the tables, which are mostly not very massive and were added to study the lower luminosity end of the star formation law. The histogram of the deconvolved FWHM sizes (angular size and linear size) of each transition are presented in Figures 26 and 27, in which again we only include massive dense clumps above the horizontal line in the tables. Figure 26 shows the histogram of the deconvolved angular FWHM size of these dense clumps, with a dashed line indicating the beam size in that transition. In HCN 3-2 maps, 29\% of dense clumps have their deconvolved FWHM sizes smaller than the beam size. For CS 7-6 maps, this ratio increases to $55 \%$, including one source (G12.21-0.10) not being resolved at all. The beam size effect can be nearly ignored for lower- $J$ transitions; almost all the FWHM sizes of HCN 1-0 and CS 2-1 are well above their beam sizes.

As Figure 27 makes particularly clear, the size distribution is skewed to low values before it is cut off by our resolution limits. Note that the plots of the lower excitation lines use a larger scale because they cover a substantially larger range of sizes.

\subsection{Luminosities}

For later comparison to work on other galaxies, we compute the line luminosities and the infrared luminosities in the same way as is done for extragalactic studies. The line luminosity of 


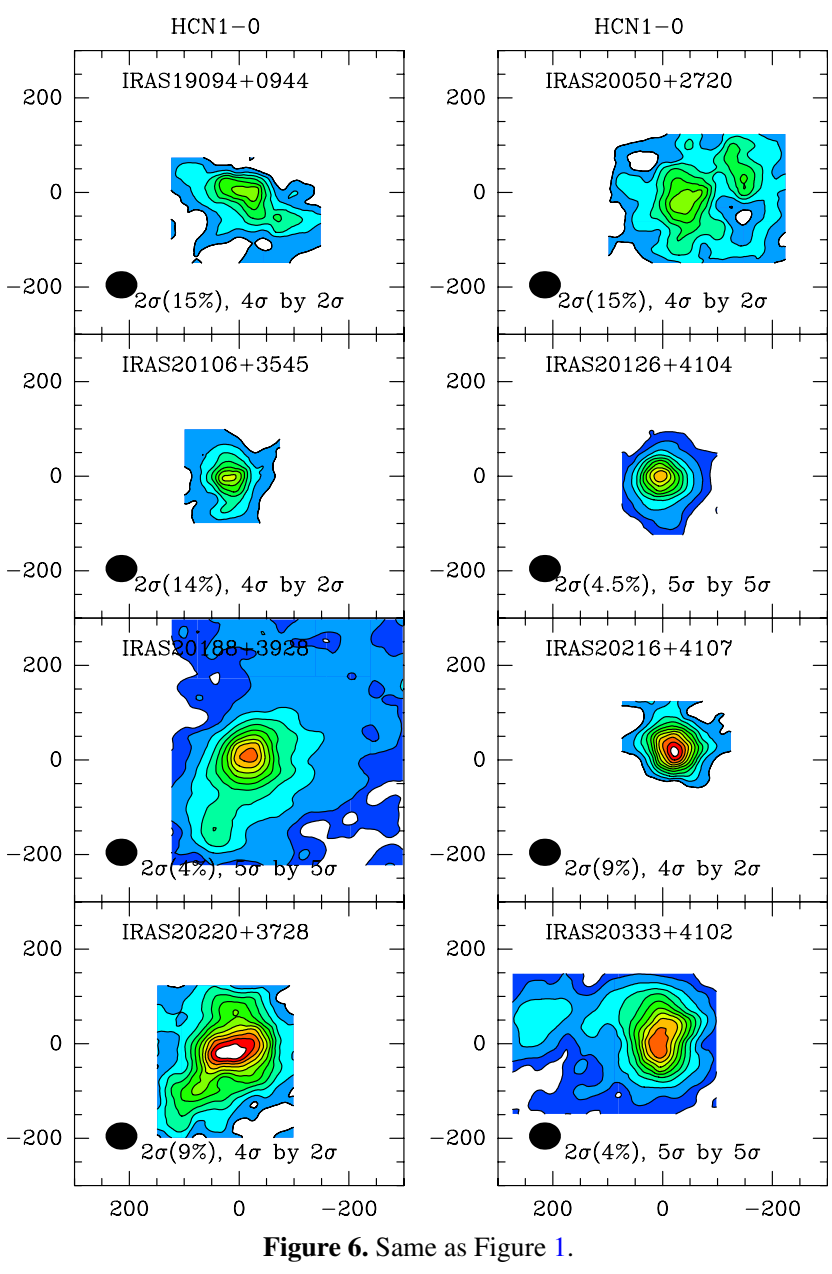

(A color version of this figure is available in the online journal.)

each core, assuming a Gaussian brightness distribution for the source and a Gaussian beam, is (we use HCN 1-0, for example)

$$
\begin{aligned}
L_{\mathrm{HCN} 1-0}^{\prime}= & 23.5 \times 10^{-6} \times D^{2} \times\left(\frac{\pi \times \theta_{s}^{2}}{4 \ln 2}\right) \times\left(\frac{\theta_{s}^{2}+\theta_{\text {beam }}^{2}}{\theta_{s}^{2}}\right) \\
& \times \int T_{R} d v \mathrm{~K} \mathrm{~km} \mathrm{~s}^{-1} .
\end{aligned}
$$

Here $D$ is the distance in kpc, and $\theta_{s}$ and $\theta_{\text {beam }}$ are the angular size of the half-peak HCN 1-0 contour and the beam in arcsecond. This method is analogous to that of Gao \& Solomon (2004b), but adapted to Galactic clumps.

The total infrared luminosity $(8-1000 \mu \mathrm{m})$ was calculated based on the four IRAS bands (Sanders \& Mirabel 1996), as was done for the galaxy sample of Gao \& Solomon (2004a):

$$
L_{\mathrm{IR}}=0.56 \times D^{2} \times\left(13.48 \times f_{12}+5.16 \times f_{25}+2.58 \times f_{60}+f_{100}\right),
$$

where $f_{x}$ is the flux in band $x$ from the four IRAS bands in units of Jy, $D$ is in kpc, and $L_{\mathrm{IR}}$ is in $L_{\odot}$. Some authors used the bolometric luminosity $\left(L_{\mathrm{bol}}\right)$, which is calculated from the spectral energy distribution (SED) of the source (e.g., Mueller et al. 2002). Our statistics show that $L_{\mathrm{bol}}$ calculated in Mueller et al. (2002) is very close to the $L_{\mathrm{IR}}$ calculated in this paper for the same clumps. The ratio of $L_{\mathrm{IR}}$ to $L_{\mathrm{bol}}$ is $1.28 \pm 0.90$, and the median is 1.09 . The relatively large dispersion for this ratio is strongly affected by two sources (S252A and CepA) whose ratio is much larger than others. Excluding these two sources,

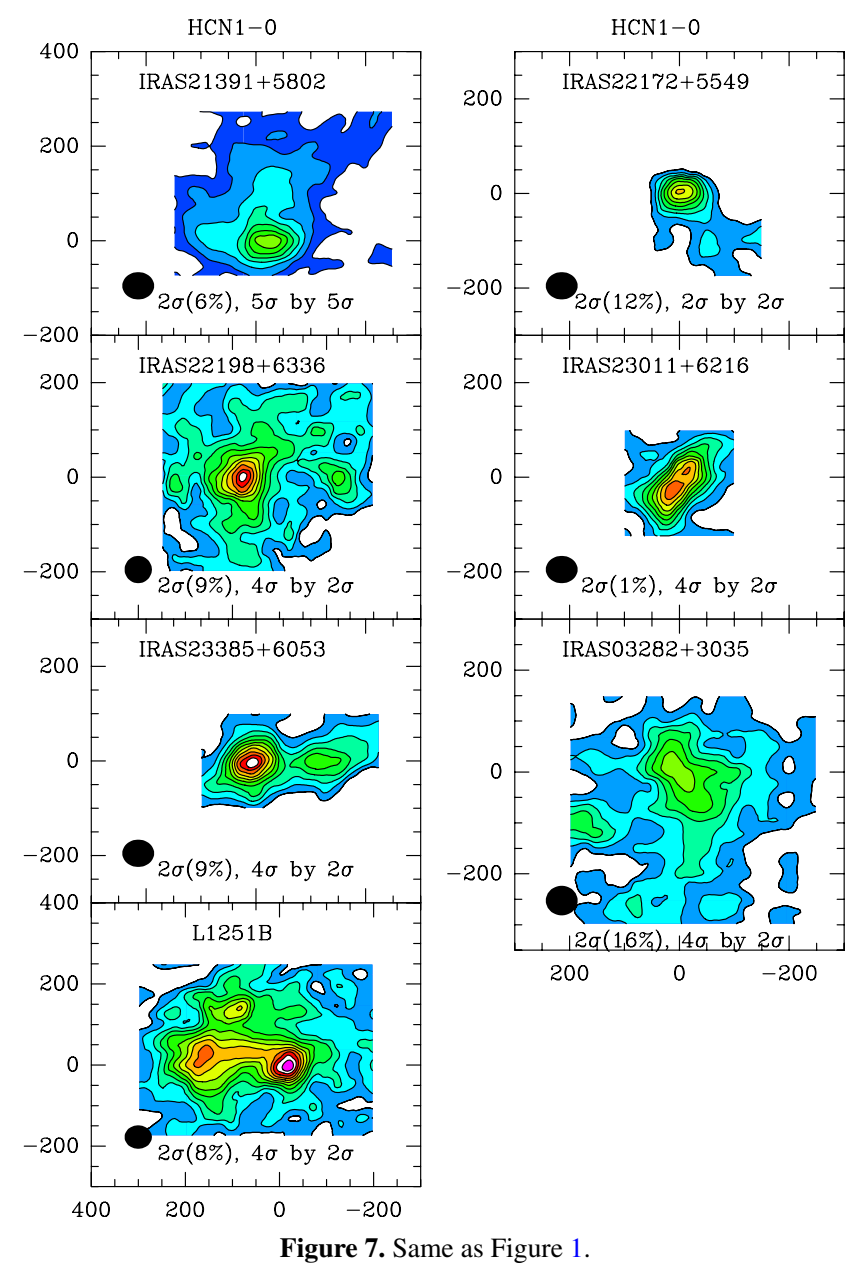

(A color version of this figure is available in the online journal.)

the mean ratio becomes $1.10 \pm 0.25$, with the median 1.08 . The mean and median values of $L_{\mathrm{IR}}$ are $(4.7 \pm 1.2) \times 10^{5} L_{\odot}$ and $1.06 \times 10^{5} L_{\odot}$, with values ranging from $708 L_{\odot}$ to $5 \times 10^{6} L_{\odot}$, considering only the sources in the original sample.

The infrared and line luminosities are tabulated in Table 7, with mean and median values for the massive, dense clumps at the bottom. The histograms of luminosity are shown in Figure 28. While the histograms appear to be well-peaked, we are systematically biased against low-luminosity sources. Together with the fact that the histograms plot the logarithm of the luminosity, the highest luminosity sources are clearly rare. For example, the mean value of luminosity is typically three times the median.

\section{DISCUSSION}

\subsection{Trends in Size with Tracer}

Although all these lines trace "dense" gas, there are differences. Higher $J$ transitions of a given species trace denser parts of the core. Crudely speaking, for a given $J$ level, HCN transitions have higher critical densities than do the corresponding transitions in CS. The dependence on temperature also differs, with the upper level of the CS 7-6 transition lying $66 \mathrm{~K}$ above ground, compared to only $26 \mathrm{~K}$ above ground for the HCN 3-2 transition.

In Table 8, we list the properties of the transitions and the statistics of the size ratios and luminosity ratios of maps derived from different tracers. Table 9 shows that maps of low- $J$ 


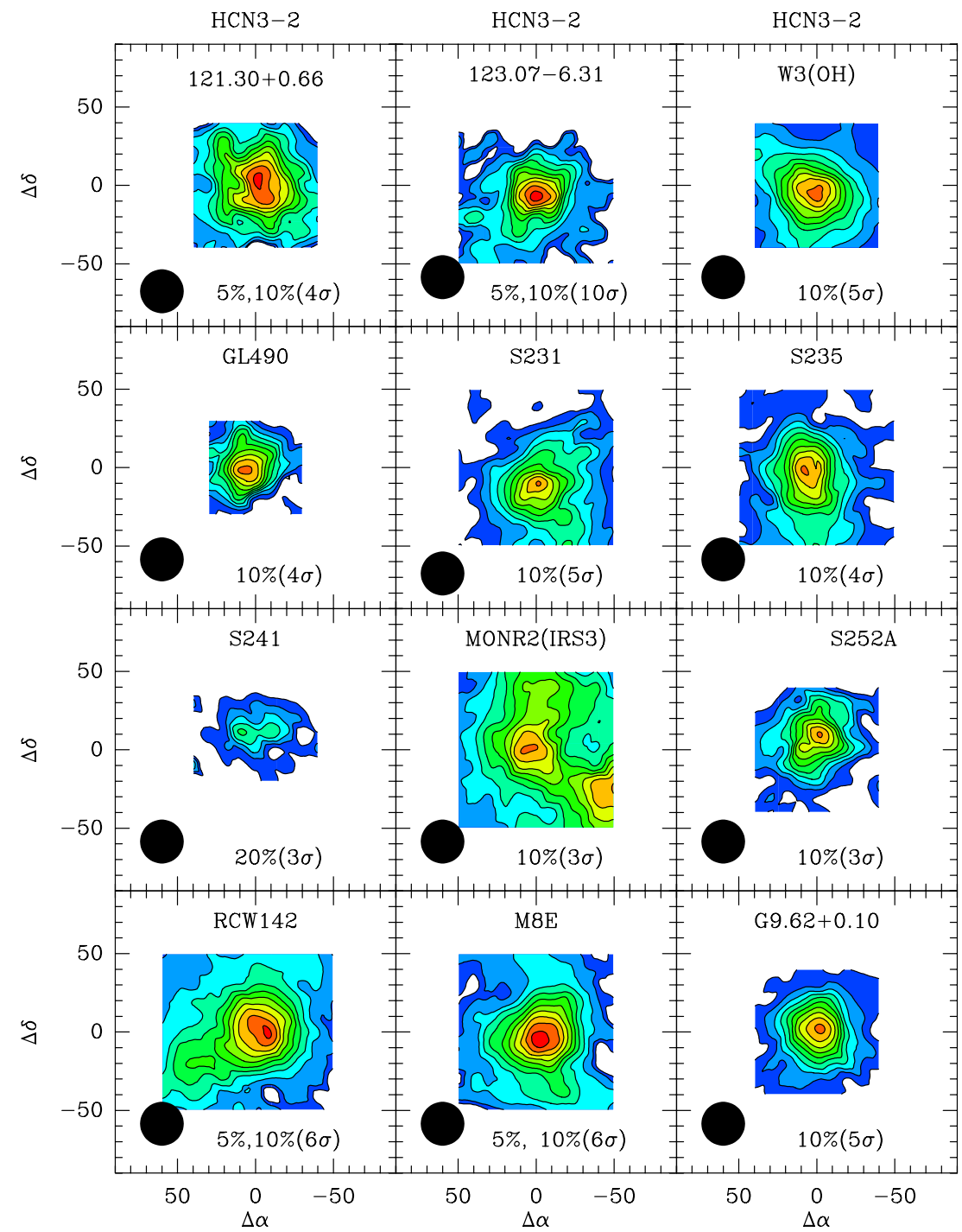

Figure 8. HCN 3-2 contour maps of massive clumps. The lowest contour level and increasing step of contours are indicated in the plot. The beam size is shown at the lower left of each map.

transitions have larger deconvolved sizes than those of higher $J$ transitions. For instance, the linear size of the HCN 3-2 map is 0.28 of the size of the HCN $1-0$ maps, and the ratio is the same for CS 7-6 versus CS 2-1.

This result would not be found in a "raisin-pudding" model of clumps: a roughly uniform pudding (the clump) with multiple dense cores sprinkled randomly throughout. That model would predict similar sizes for maps of transitions sensitive to different density regimes. Such a pattern was seen in early studies of multiple transitions in a small number of sources (Snell et al. 1984; Mundy et al. 1986, 1987). The current results have higher spatial resolution and more complete maps in the high-excitation lines than the early results and do show that higher excitation lines are more centrally condensed. If the clumps are centrally condensed, with either a smooth increase in density with decreasing radius, or an increased fraction of the volume filled by dense "raisins," the differences in size would be predicted as the lines from higher- $J$ transitions would be strongly enhanced in the denser regions. Detailed models of individual dense clumps do support density gradients with power laws providing good fits $\left(n(r) \propto r^{-p}\right.$ with $\langle p\rangle=1.8$; Mueller et al. 2002).

\subsection{An Inverse Size-Linewidth Relation}

The linewidth at the peak of the dense clumps for different tracers shows an interesting trend. As presented in Table 10, the mean and median FWHM linewidth of the dense clumps increase for higher- $J$ transitions, from about $4.8 \mathrm{~km} \mathrm{~s}^{-1}$ for CS $2-1$, to about $5.9 \mathrm{~km} \mathrm{~s}^{-1}$ for CS 7-6. If we look at a optically thin tracer, like $\mathrm{C}^{34} \mathrm{~S} 5-4$, the mean FWHM is $4.7 \mathrm{~km} \mathrm{~s}^{-1}$, which is closer to that of the CS 2-1 than higher- $J$ CS transitions.

Studies of molecular clouds have established a positive correlation of cloud size, as mapped with a single tracer, and linewidth of that tracer (Larson 1981; Myers 1985), which can be interpreted in terms of the virial theorem. The linewidth-size relation within individual cores or clumps is much less wellestablished (e.g., Evans 1999). Here we refer to the useful distinction introduced by Goodman et al. (1998) between four different kinds of relationships. A Goodman Type-4 relation plots linewidths of a single tracer measured over regions of increasing radius; such plots show little correlation (Goodman et al. 1998; Barranco \& Goodman 1998). Goodman Type-1 relations plot sizes measured in multiple tracers for multiple cores or clumps. Such plots generally show positive correlations 


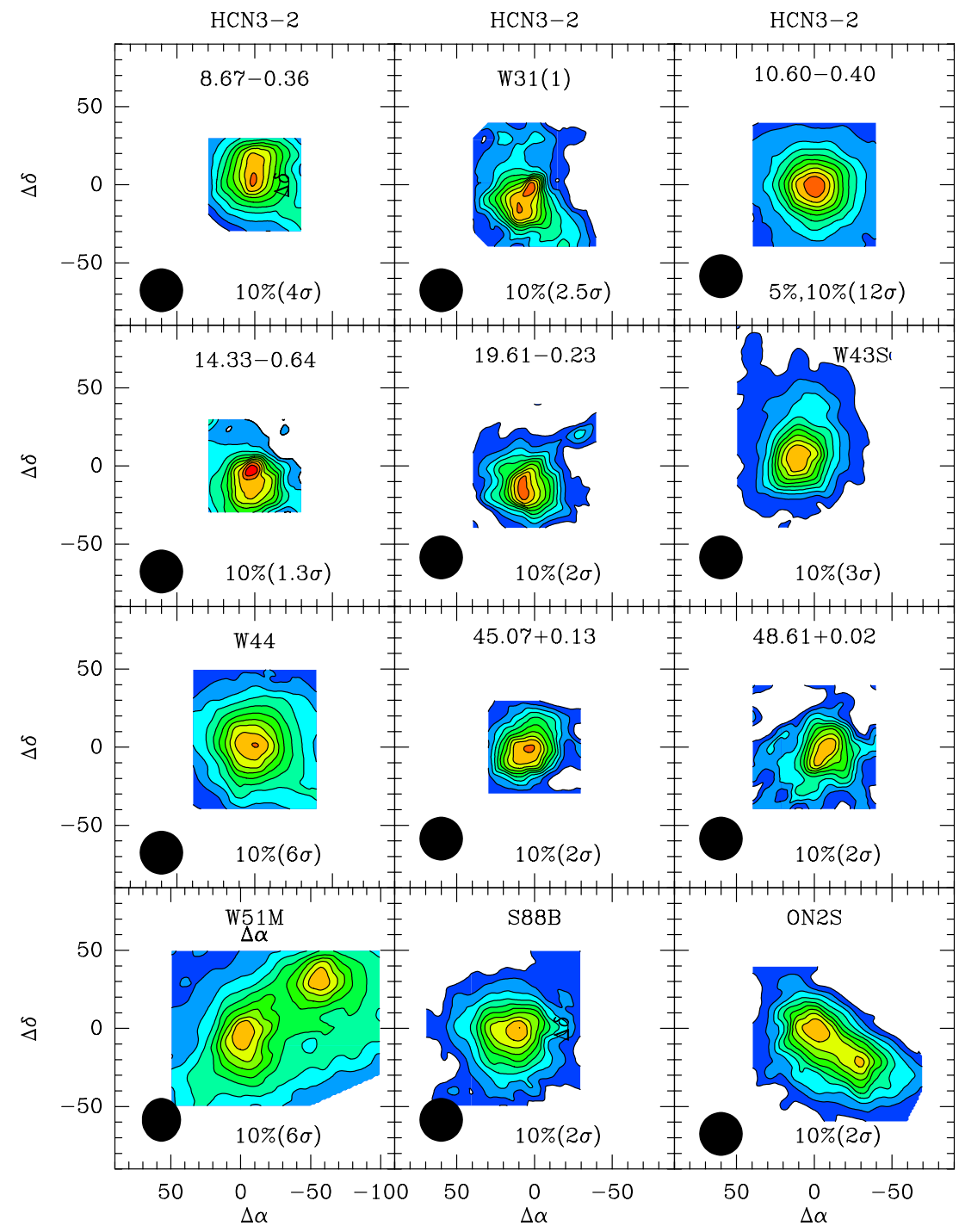

Figure 9. Same as Figure 8.

(A color version of this figure is available in the online journal.)

when using tracers of modest density applied to low-mass and intermediate-mass cores or clumps; for example, Caselli \& Myers (1995) found $\Delta v \propto r^{0.53 \pm 0.7}$ with a correlation coefficient of 0.81 using $\mathrm{NH}_{3}$, CS 2-1, and $\mathrm{C}^{18} \mathrm{O} 1-0$ maps of eight starless cores.

In contrast, studies of the cores in this sample with Goodman Type-2 relations (single tracer, multicore) have shown no correlation between linewidth and size (Plume et al. 1997 for CS 7-6) or a weak $(r=0.43)$ correlation (Shirley et al. 2003 for $\left.\mathrm{C}^{34} \mathrm{~S} 5-4\right)$. In both cases, the linewidths were 4-5 times larger than would be predicted from the usual linewidth-size relations developed from studies of clouds and low-mass cores. With maps of multiple tracers toward many cores, we could construct Goodman Type-3 relations (multitracer, single core) for each core. Instead, we try a modified version (Type 5?) in which we use the mean value over many cores for each transition.

Figure 29 shows the mean linewidth versus the mean size of the dense clumps for CS 2-1, CS 5-4, CS 7-6, and HCN 1-0 transitions. HCN 3-2 lines have serious self-absorption, therefore they are excluded from this figure. HCN $1-0$ has three hyperfine components; we took the mean linewidth of the strongest component $(F=2-1)$ for the plot. The mean linewidth of the tracer has an inverse correlation with the mean size of the dense clumps. For example, while the CS 2-1 sizes are on average four times larger than those of CS 7-6, the mean of the linewidth ratio of CS 7-6 to CS $2-1$ is $1.18 \pm$ 0.34 for the massive dense clumps; the median ratio is 1.16 . The CS 2-1 emission traces a larger, quieter area than higher$J$ transitions, while CS 5-4 and CS 7-6 trace more compact and apparently more turbulent regions. Alternatively, the larger linewidths toward the center could in part reflect higher inflow motions. A similar trend was seen in observations and models of G10.6 (Keto 1990). It is also possible that the turbulence (here refers to all unresolved gas motions, including inflow, outflow, complex fluid motions, etc.) has radial structure, which is higher close to the forming protostars and therefore is being picked up better by the higher- $J$, dense gas tracers. The usual size-linewidth relations certainly do not apply to these regions where massive stars are forming.

\subsection{What Physical Conditions Do the Lines "Trace"?}

While we speak of a transition as "tracing" gas of a certain density, this formulation is too simplistic; the emission from a transition increases smoothly and strongly over a wide range of 


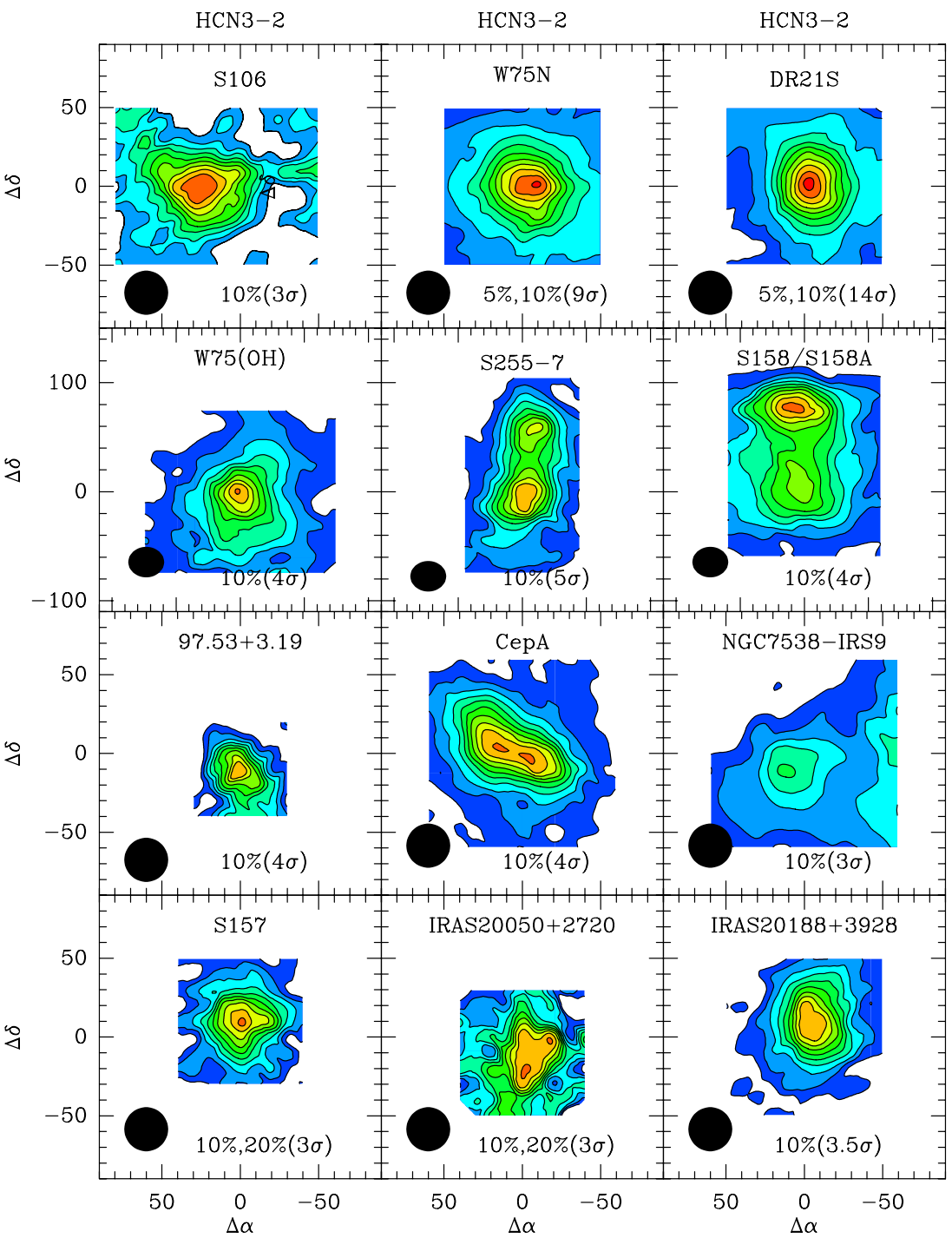

Figure 10. Same as Figure 8.

(A color version of this figure is available in the online journal.)

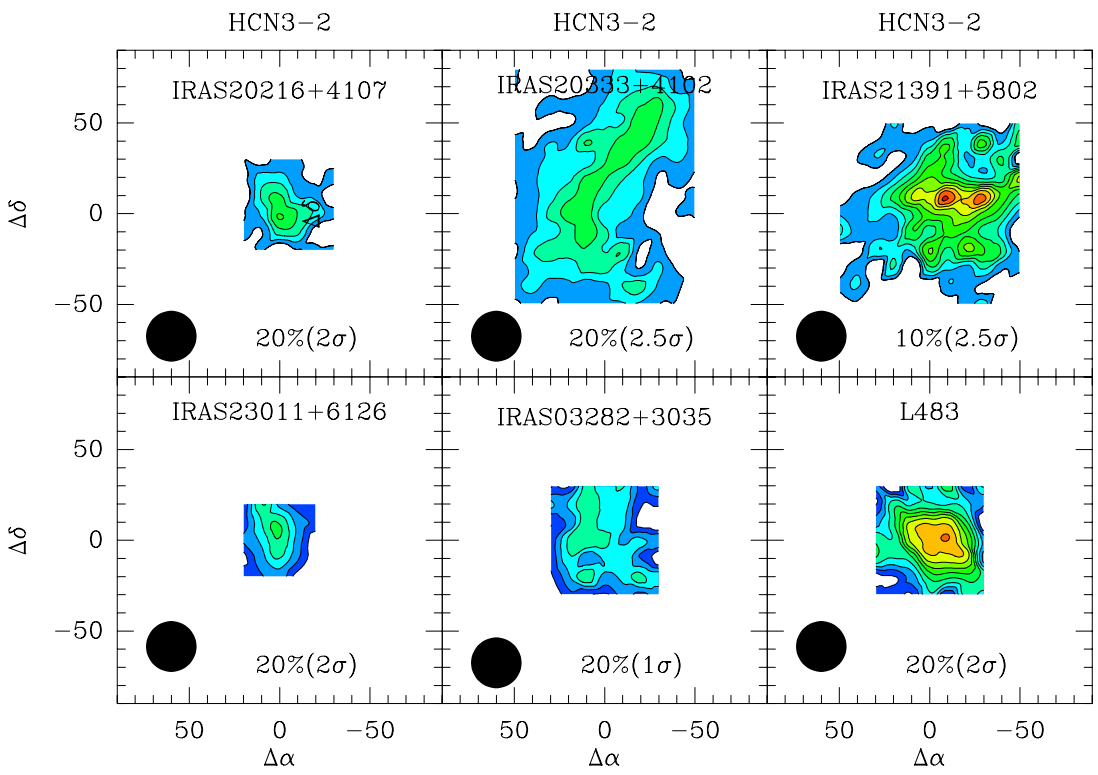

Figure 11. Same as Figure 8.

(A color version of this figure is available in the online journal.) 


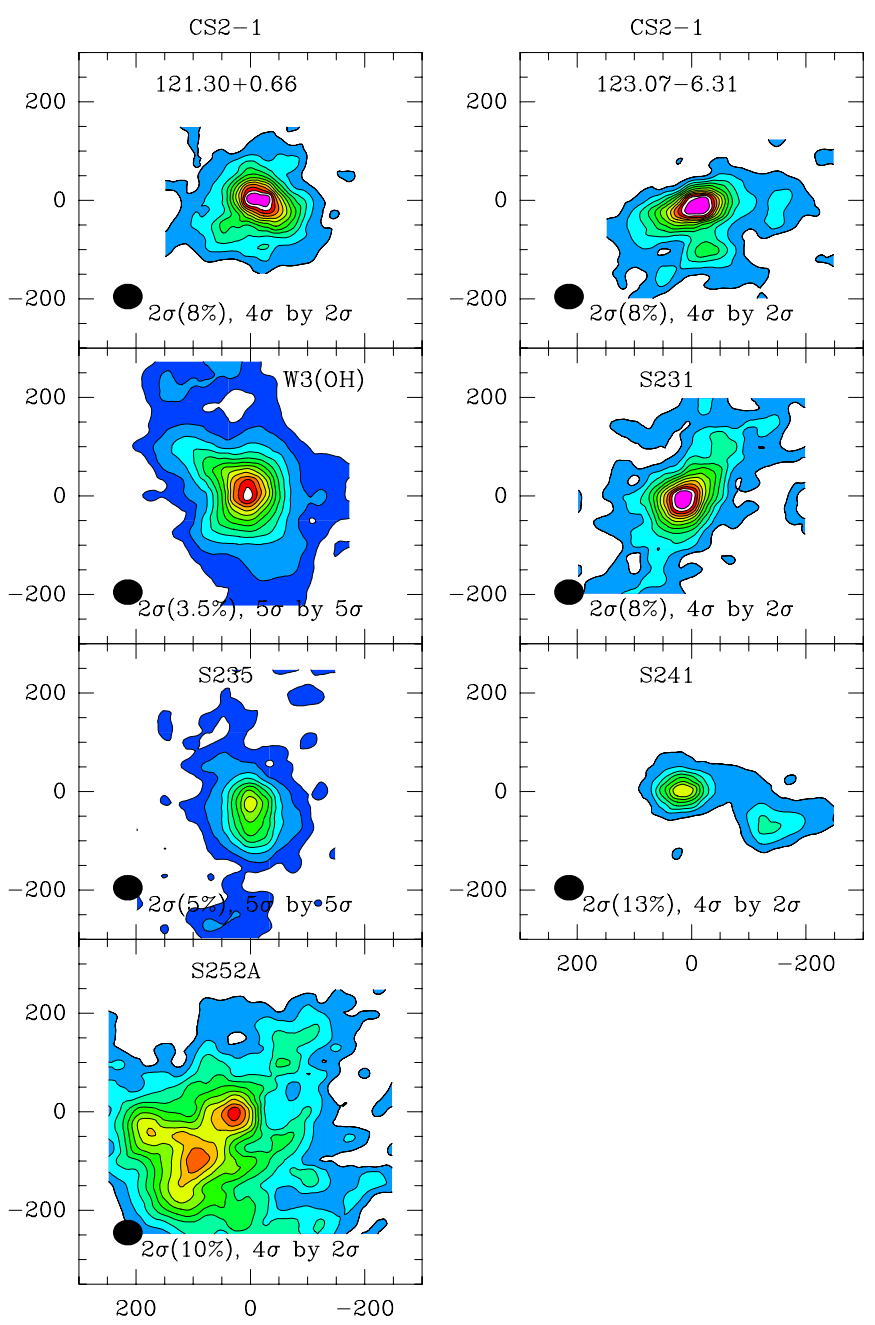

Figure 12. CS 2-1 contour maps of massive clumps. The lowest contour level and increasing step of contours are indicated in the plot. The beam size is shown at the lower left of each map.

(A color version of this figure is available in the online journal.)

densities and then levels out just above the critical density. (This description applies only to lines with frequencies high enough that radiative transitions stimulated by cosmic background radiation or continuum emission from dust are unimportant; Evans 1989.) Nonetheless, it is common to speak of a transition as tracing its critical density. If anything, the opposite is true, as transitions fail to provide useful probes of density above their critical density. A more sensible fiducial density is the effective density (Evans 1999), which is the density needed to produce a line with a radiation temperature of $1 \mathrm{~K}$ for typical conditions. For these comparisons, we use the critical and effective densities calculated for a kinetic temperature of $100 \mathrm{~K}$ (Evans 1999). Both these measures are given in Table 9.

The critical densities $\left(\mathrm{n}_{\text {crit }}\right)$ of the HCN 1-0 and CS 2-1 lines differ by an order of magnitude, but their effective densities $\left(\mathrm{n}_{\text {eff }}\right)$, are very similar. The similarity of their mean sizes indicates that $n_{\text {eff }}$ better reflects the excitation requirements. For HCN 3-2, CS 5-4, and CS 7-6, their sizes are also similar while the critical and effective densities make opposite predictions; CS 7-6 has a much higher $n_{\text {eff }}$ than the other two, but HCN 3-2 has the highest $n_{\text {crit }}$. The excitation energy of the upper level $\left(E_{\text {up }}\right)$ is considerably higher for the CS 7-6 transition. If we consider only the sources that have been mapped by all three high- $J$
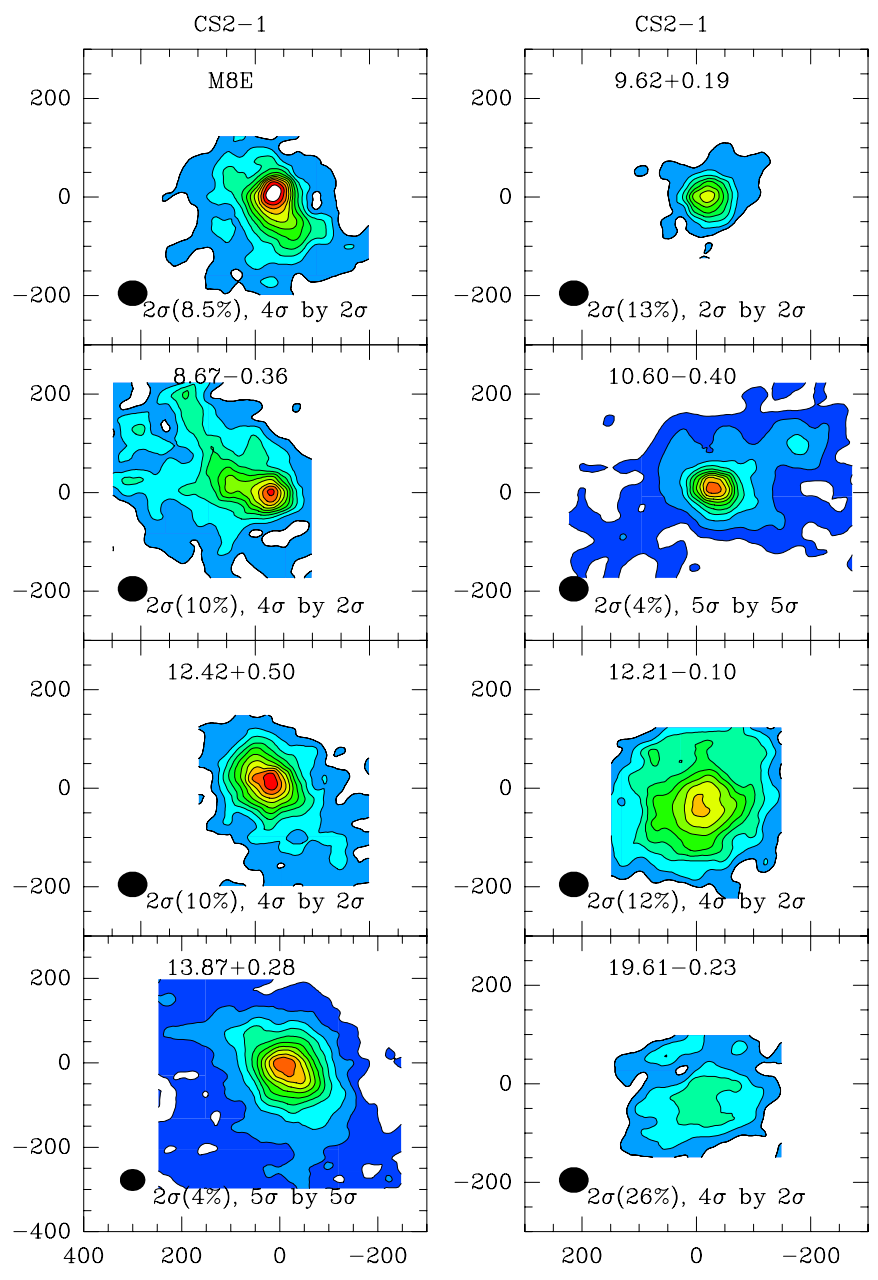

Figure 13. Same as Figure 12.

(A color version of this figure is available in the online journal.)

transitions CS 7-6, CS 5-4, and HCN 3-2 (Columns 5 and 7 in Table 9), statistics show that the mean size and luminosity of the dense clumps increase with decreasing $E_{\text {up }}$ of the transitions, as well as decreasing $n_{\text {eff }}$, while the correlation with $n_{\text {crit }}$ is weaker. The tracers that need higher excitation energy or density are confined to smaller regions in the dense clumps, consistent with centrally condensed structures. In addition, $n_{\text {eff }}$ better reflects the excitation requirement than $n_{\text {crit }}$.

Empirically, we find that the HCN 1-0 and CS 2-1 lines form a matched set, while $\mathrm{HCN} 3-2$ and CS 5-4 lines make another pair; each pair has very similar sizes and fairly similar luminosities in the mean. The CS 7-6 lines are close to the properties of the HCN 3-2 and CS 5-4 pair, but still more compact in size.

\subsection{Virial Mass, Surface Density, Volume Density, and Relations with $L_{I R}$}

The virial mass of the dense clumps with power-law density distributions $\left(n(r) \propto r^{-p}\right)$ is calculated by

$$
\begin{gathered}
M_{\mathrm{Vir}}(R)=\frac{5 R \Delta v^{2}}{8 a_{1} a_{2} G \ln 2} \approx 209 \frac{(R / 1 \mathrm{pc})\left(\Delta v / 1 \mathrm{~km} \mathrm{~s}^{-1}\right)^{2}}{a_{1} a_{2}} M_{\odot} \\
a_{1}=\frac{1-p / 3}{1-2 p / 5}, p<2.5
\end{gathered}
$$




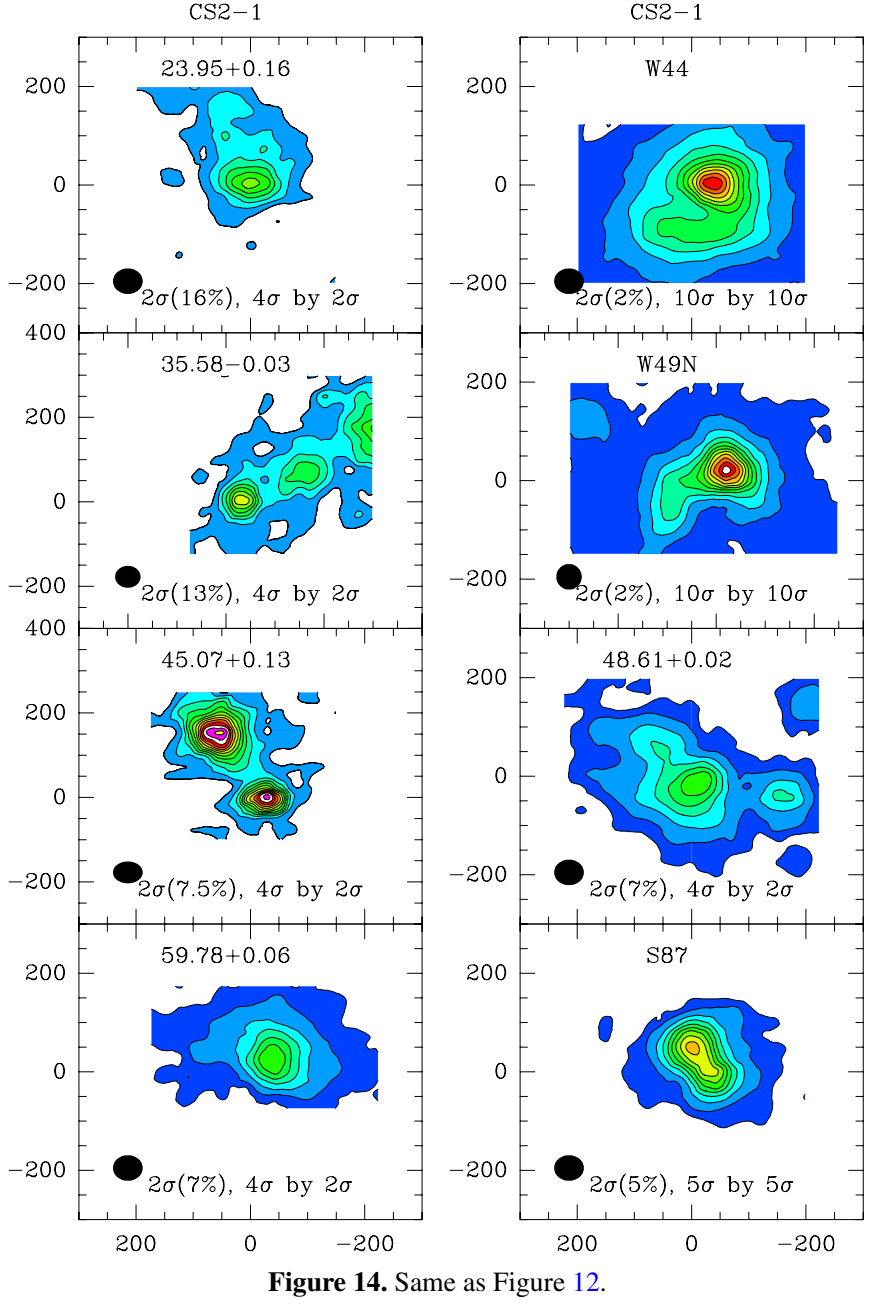

(A color version of this figure is available in the online journal.)

where $a_{1}$ is the correction for a power-law density distribution and $a_{2}$ is the correction for a nonspherical shape (Bertoldi \& McKee 1992). For aspect ratios less than 2, $a_{2} \sim 1$ and can be ignored for our sample. To calculate $a_{1}$ we take the average $p$ value (1.77) from Mueller et al. (2002) for all massive dense clumps. A detailed discussion of calculating the virial mass of dense clumps in this sample can be found in Shirley et al. (2003).

The virial masses calculated from each transition $\left(M_{\text {Vir }}\left(R_{\text {transition }}\right)\right)$, for HCN 1-0, 3-2, CS 7-6, and 2-1, are tabulated in Table 11. Rather than using the linewidth of the tracer, we used the linewidth of $C^{34} S 5-4$ for all these tracers because it is more reliably optically thin than is the $3-2$ line of $\mathrm{H}^{13} \mathrm{CN}$. For lower mass sources (those below the horizontal line in Table 11), their $\mathrm{C}^{34} \mathrm{~S} 5-4$ lines are too weak to detect or to give a Gaussian fit except for IRAS20188+3928. The FWHM linewidth of CS 2-1 is closest to that of $\mathrm{C}^{34} \mathrm{~S} 5-4$ in our sample, with a ratio of linewidth for CS 2-1 to $\mathrm{C}^{34} \mathrm{~S} 5-4$ of $1.2 \pm 0.1$ and a median of 1.1. So, for these lower mass clumps, we used the CS 2-1 linewidth to calculate their virial mass, and assigned an additional $20 \%$ uncertainty to their linewidth uncertainties. While the linewidths vary among transitions by about $25 \%$, some of this variation must be caused by optical depth differences, and the differences in any case, are small. The sense of these calculations is then an estimate of the mass within the FWHM contour of each tracer. The mean ratio of $M_{\mathrm{vir}}$ for CS 2-1 to CS 7-6 is 4.0 , with a median of 3.7 ; the mean ratio for $\mathrm{HCN} 1-0$ and 3-2
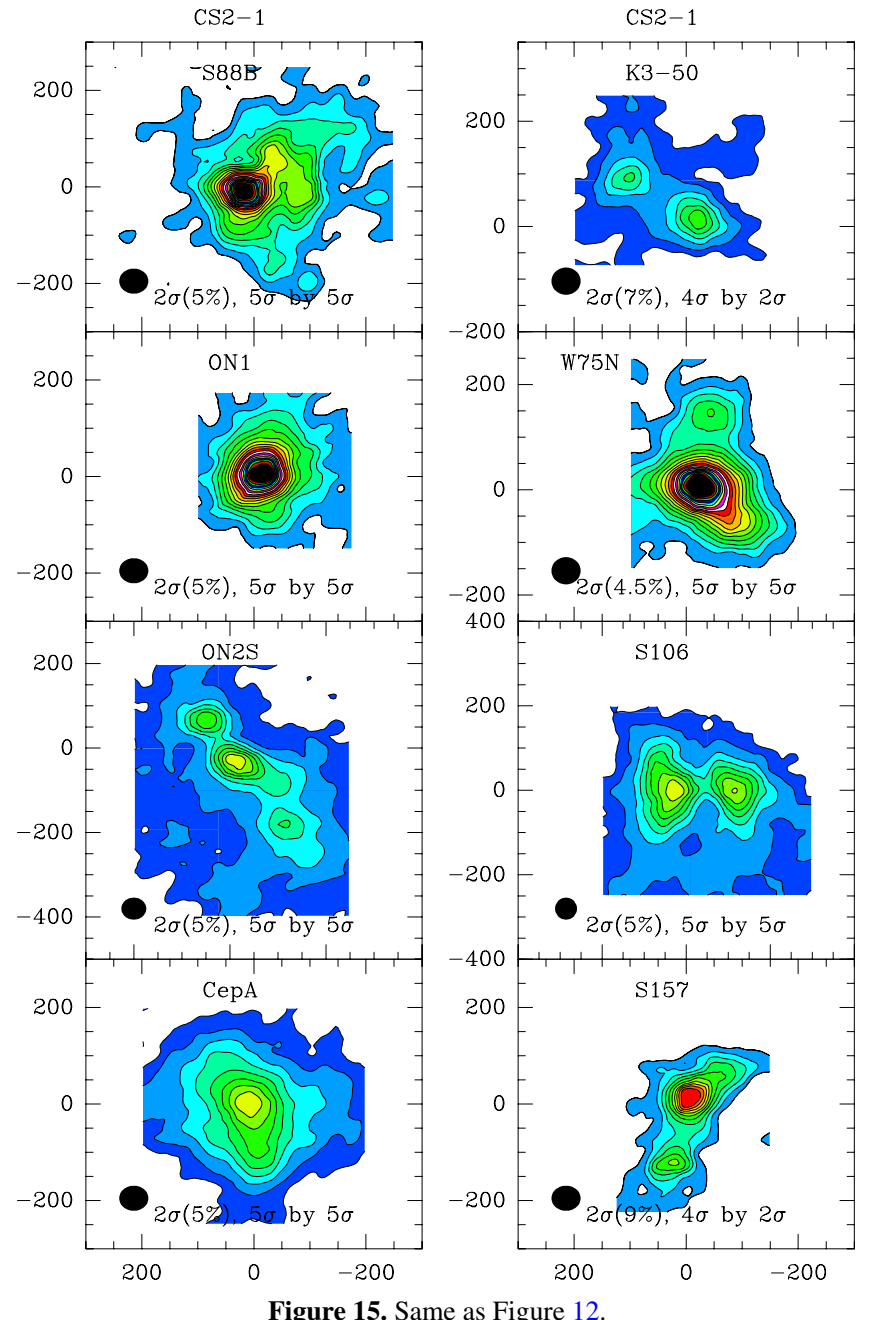

(A color version of this figure is available in the online journal.)

is 2.9 , with a median of 2.8. One would expect these ratios to reflect the size ratios, which are 4.1 and 3.0 (the mean ratio), respectively.

The virial mass enclosed by the FWHM contour of dense gas tracers represents the mass, most of which is dense gas, that is available to clustered star formation. Therefore, one should expect a positive correlation between the star formation rates, as indicated by the infrared luminosity, and the virial mass. One caveat is that the star formation rate can be underestimated by $L_{\mathrm{IR}}$ until the initial mass function (IMF) is well sampled by the star formation process (Krumholz \& Thompson 2007). We plot $M_{\mathrm{Vir}}$ versus $L_{\mathrm{IR}}$ for four transitions in Figure 30 . It is obvious that the infrared luminosity is positively correlated with the virial mass for these clumps. We used linear fits based on least squares minimization and alternatively on robust estimation, in which the absolute value of the deviation is minimized. Comparing these two fits usually provides a more realistic appraisal of the true uncertainties.

As noted by $\mathrm{Wu}$ et al. (2005), there is a cutoff around $L \sim 10^{4.5} L_{\odot}$ in the $L_{\mathrm{IR}}-L_{\mathrm{HCN} 1-0}^{\prime}$ correlation. If we fit only the data for massive dense clumps with $L_{\mathrm{IR}}>10^{4.5} L_{\odot}$, the fitting results are as follows ( $r$ is the correlation coefficient):

HCN 1-0:

Least squares $: \log \left(L_{\mathrm{IR}}\right)=1.29( \pm 0.08) \times \log \left(M_{\mathrm{Vir}}\left(R_{\mathrm{HCN} 1-0}\right)\right)$ $+0.98( \pm 0.28) ; r=0.69$ 


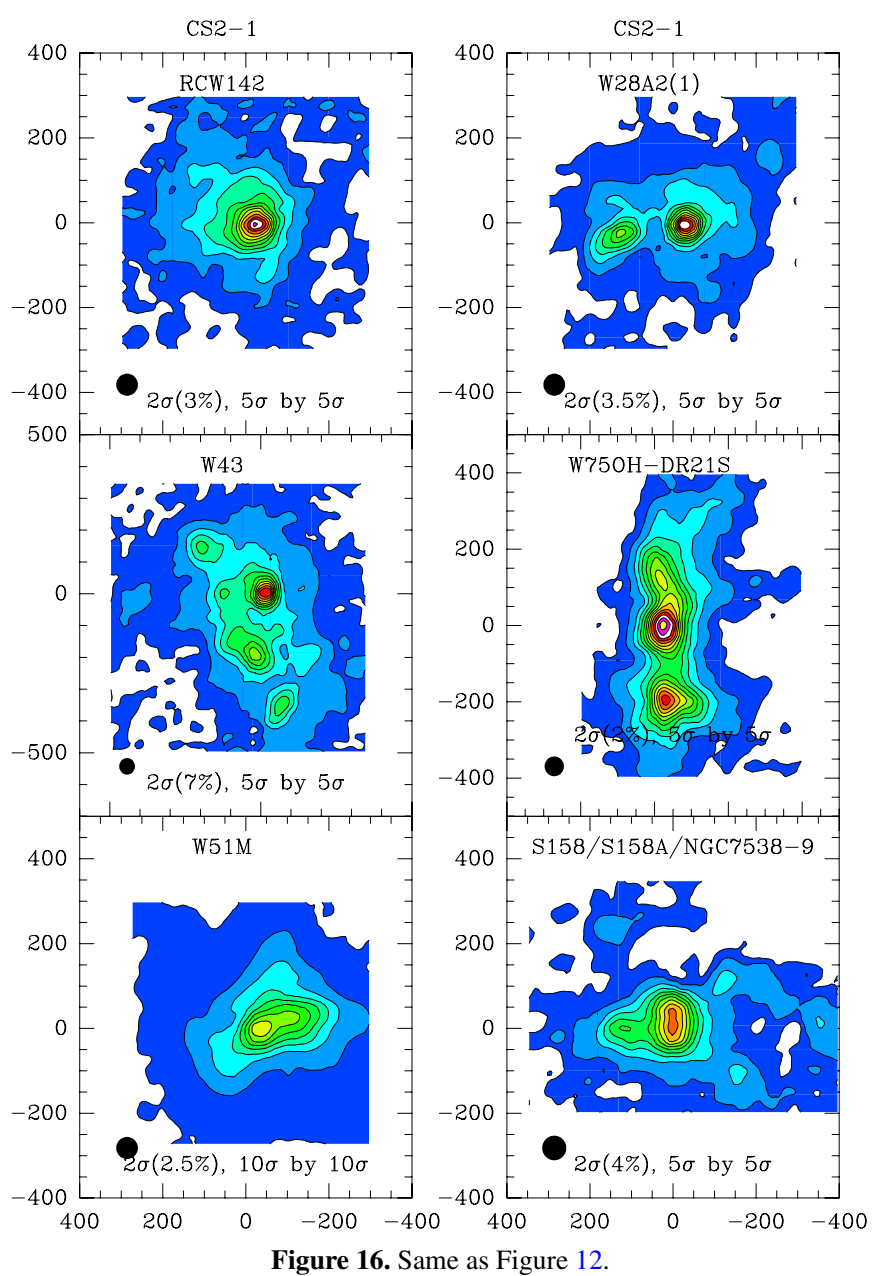

(A color version of this figure is available in the online journal.)

Robust fit $: \log \left(L_{\mathrm{IR}}\right)=0.70 \times \log \left(M_{\mathrm{Vir}}\left(R_{\mathrm{HCN} 1-0}\right)\right)+3.03$

\section{HCN 3-2:}

Least squares : $\log \left(L_{\mathrm{IR}}\right)=1.23( \pm 0.09) \times \log \left(M_{\mathrm{Vir}}\left(R_{\mathrm{HCN} 3-2}\right)\right)$

$$
+1.98( \pm 0.24) ; r=0.61
$$

Robust fit : $\log \left(L_{\mathrm{IR}}\right)=0.79 \times \log \left(M_{\mathrm{Vir}}\left(R_{\mathrm{HCN} 3-2}\right)\right)+3.16$

\section{CS 2-1:}

Least squares $: \log \left(L_{\mathrm{IR}}\right)=1.13( \pm 0.06) \times \log \left(M_{\mathrm{Vir}}\left(R_{\mathrm{CS} 2-1}\right)\right)$

$$
+1.61( \pm 0.19) ; r=0.67
$$

Robust fit : $\log \left(L_{\mathrm{IR}}\right)=0.98 \times \log \left(M_{\mathrm{Vir}}\left(R_{\mathrm{CS} 2-1}\right)\right)+2.24$

\section{CS 7-6:}

Least squares $: \log \left(L_{\mathrm{IR}}\right)=1.42( \pm 0.07) \times \log \left(M_{\mathrm{Vir}}\left(R_{\mathrm{CS} 7-6}\right)\right)$

$$
+1.28( \pm 0.23) ; r=0.49
$$

Robust fit : $\log \left(L_{\mathrm{IR}}\right)=0.62 \times \log \left(M_{\mathrm{Vir}}\left(R_{\mathrm{CS} 2-1}\right)\right)+3.76$.

There are substantial variations between fitting method employed and among the line used. If we average the two fitting
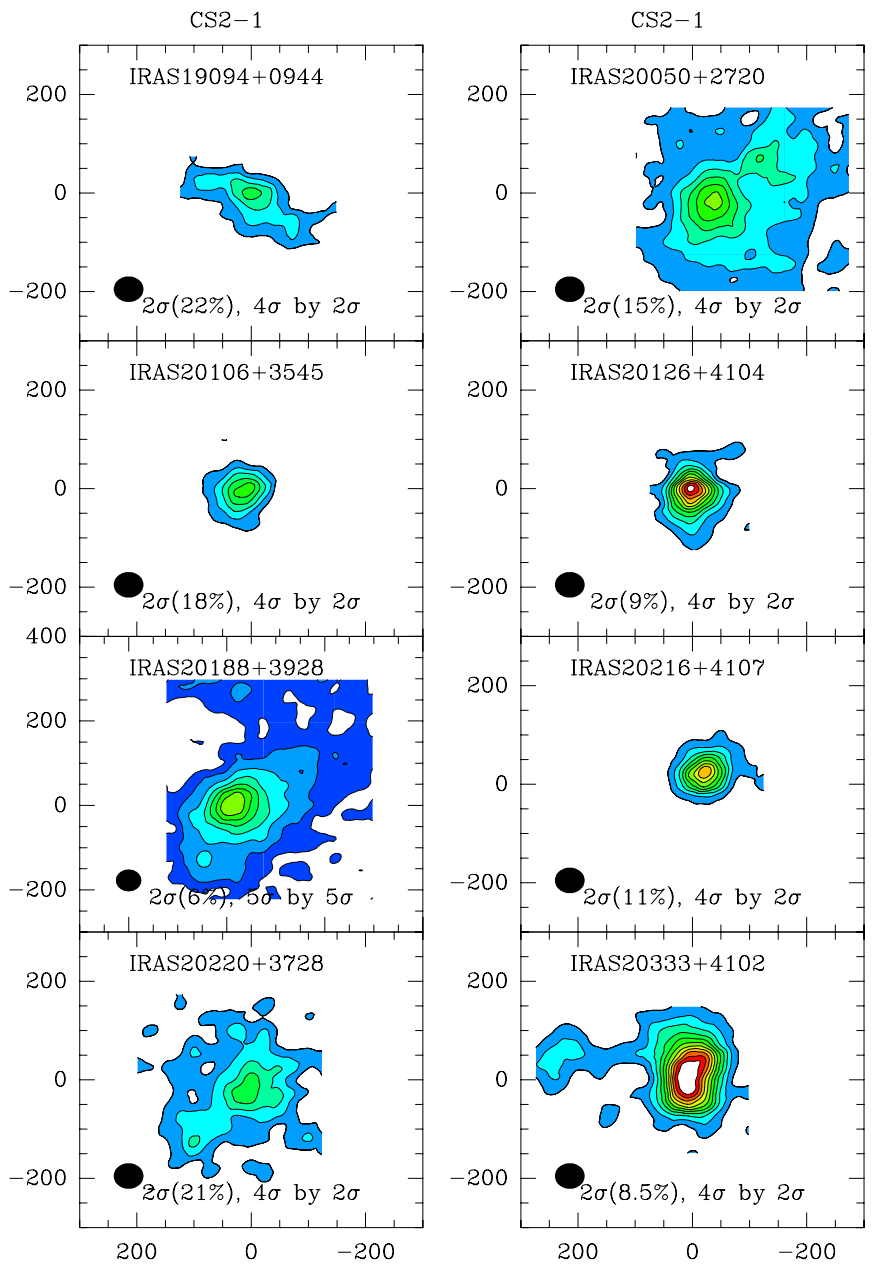

Figure 17. Same as Figure 12.

(A color version of this figure is available in the online journal.)

methods for each line and then average over all lines, the resulting slope is $1.02 \pm 0.03$. Thus, $L_{\mathrm{IR}}$ is roughly linearly correlated with the virial mass for dense clumps with $L_{\mathrm{IR}}>10^{4.5} L_{\odot}$.

Below this threshold, the slope is steeper. We also provide linear fits and robust fits to the sources with $L_{\mathrm{IR}}<10^{4.5} L_{\odot}$ (the CS 7-6 maps have too few sources below this luminosity to make a reasonable fitting):

HCN 1-0:

Least Squares : $\log \left(L_{\mathrm{IR}}\right)=2.02( \pm 0.13) \times \log \left(M_{\mathrm{Vir}}\left(R_{\mathrm{HCN} 1-0}\right)\right)$

$$
-1.43( \pm 0.30) ; r=0.87
$$

Robust Fit $: \log \left(L_{\mathrm{IR}}\right)=1.71 \times \log \left(M_{\mathrm{Vir}}\left(R_{\mathrm{HCN} 1-0}\right)\right)-0.81$

HCN 3-2:

Least squares : $\log \left(L_{\mathrm{IR}}\right)=2.30( \pm 0.25) \times \log \left(M_{\mathrm{Vir}}\left(R_{\mathrm{HCN} 3-2}\right)\right)$

$$
-0.98( \pm 0.53) ; r=0.83
$$

Robust fit : $\log \left(L_{\mathrm{IR}}\right)=0.97 \times \log \left(M_{\mathrm{Vir}}\left(R_{\mathrm{HCN} 3-2}\right)\right)+1.69$

CS 2-1:

Least squares : $\log \left(L_{\mathrm{IR}}\right)=2.46( \pm 0.23) \times \log \left(M_{\mathrm{Vir}}\left(R_{\mathrm{CS} 2-1}\right)\right)$

$$
-2.44( \pm 0.56) ; r=0.87
$$




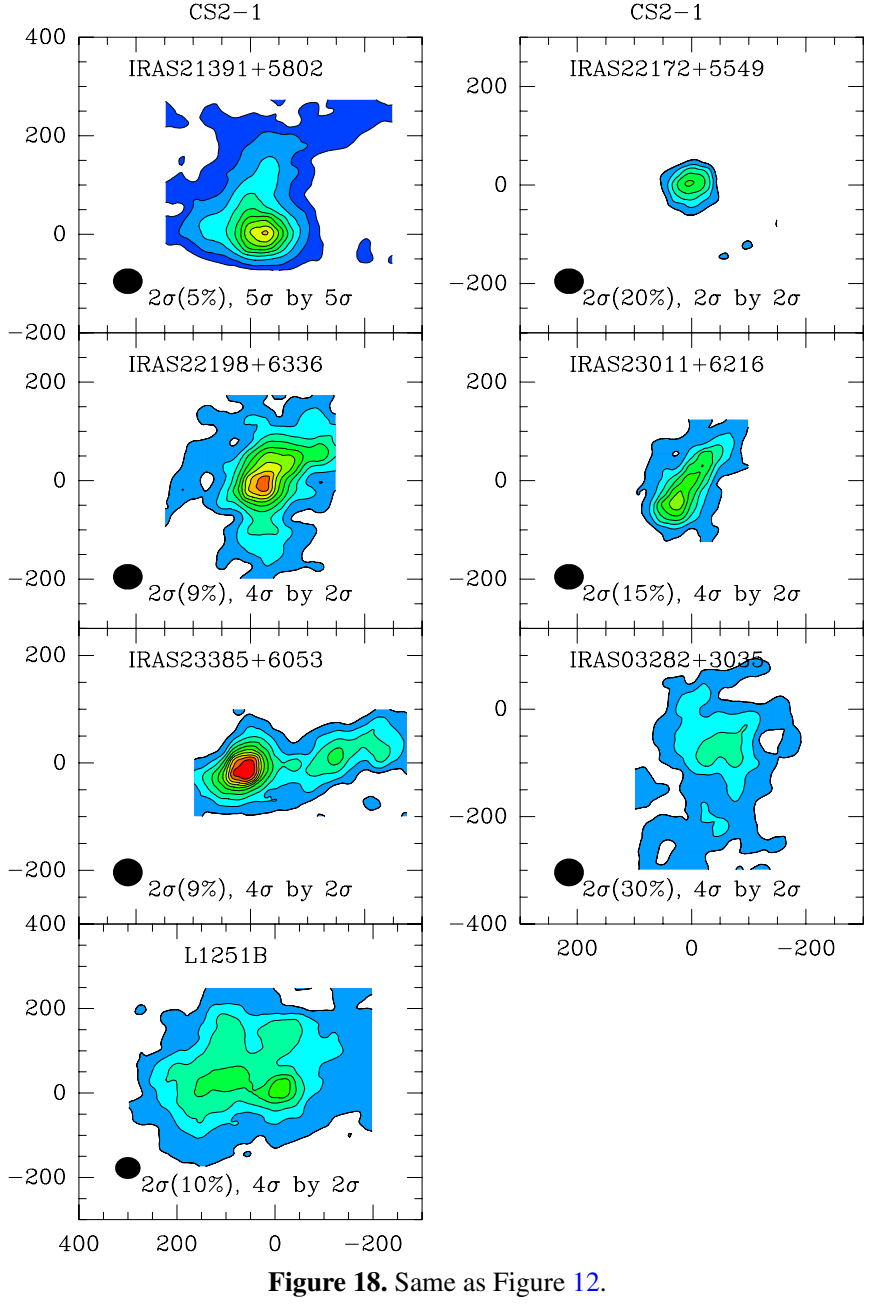

(A color version of this figure is available in the online journal.)

Robust fit $: \log \left(L_{\mathrm{IR}}\right)=1.59 \times \log \left(M_{\mathrm{Vir}}\left(R_{\mathrm{CS} 2-1}\right)\right)-0.68$.

When only clumps with $L_{\mathrm{IR}}<10^{4.5}$ are included in the fit, the $L_{\mathrm{IR}}$ increases steeply with the mass of dense gas with a power law ranging from 1.5 to 2.5 . Using the same averaging method yields a mean slope of $1.84 \pm 0.20$.

The surface density of a molecular clump should have an important impact on the timescale and mass accretion rate of star formation in the dense clump (McKee \& Tan 2002, 2003). Recent work by Krumholz \& McKee (2008) proposed that only clouds with column densities of at least $1 \mathrm{~g} \mathrm{~cm}^{-2}$ can form massive stars. We can test this idea on these dense clumps with different dense gas tracers. We calculate the surface density within the FWHM size of each transition, following the method used by Shirley et al. (2003) for CS 5-4:

$$
\Sigma_{\text {transition }}=\frac{M_{\text {vir }}\left(R_{\text {transition }}\right)}{\pi R_{\text {transition }}^{2}} \approx 0.665 \frac{\left(M_{\text {vir }} / 1.0 \times 10^{4} M_{\odot}\right)}{\left(R_{\text {transition }} / 1 \mathrm{pc}\right)^{2}} .
$$

The calculated surface densities are listed in Table 11. The surface densities are lower than $1 \mathrm{~g} \mathrm{~cm}^{-2}$ within the FWHM size of CS 2-1 and HCN 1-0 maps. For massive dense clumps, the mean is $0.29 \mathrm{~g} \mathrm{~cm}^{-2}$ for HCN $1-0$, with a median $0.28 \mathrm{~g} \mathrm{~cm}^{-2}$, and $0.33 \mathrm{~g} \mathrm{~cm}^{-2}$ for CS $2-1$, with a median $0.31 \mathrm{~g} \mathrm{~cm}^{-2}$. Almost no source has surface density greater than $1 \mathrm{~g} \mathrm{~cm}^{-2}$, when measured by HCN $1-0$ or CS $2-1$. Higher- $J$ transitions trace more compact, denser regions, resulting a larger surface densities. The mean surface density within the FWHM contour of HCN 3-2 is $0.78 \pm 0.55 \mathrm{~g} \mathrm{~cm}^{-2}$, with a median of $0.68 \mathrm{~g} \mathrm{~cm}^{-2}$. This result is similar to the result from CS 5-4 maps (the mean is $0.82 \mathrm{~g} \mathrm{~cm}^{-2}$, with a median of $0.60 \mathrm{~g} \mathrm{~cm}^{-2}$; Shirley et al. 2003). CS 7-6 traces the most compact part of the clumps in this survey, and its surface density is closest to the theoretical threshold of $1 \mathrm{~g} \mathrm{~cm}^{-2}$. The mean surface density within CS 7-6 clumps is $1.09 \mathrm{~g} \mathrm{~cm}^{-2}$, with a median of $0.89 \mathrm{~g} \mathrm{~cm}^{-2}$. The percentage of sources that have surface density larger than $1 \mathrm{~g} \mathrm{~cm}^{-2}$ is $26 \%, 31 \%$, and $38 \%$ for clumps traced by CS $5-4$, HCN 3-2, and CS 7-6, respectively.

As one might have expected, tracers with higher $n_{\text {eff }}$ trace higher surface densities. Do the surface densities correlate with other properties? One might expect that the higher the surface density, the larger the virial mass of the clumps within the same FWHM area. According to Krumholz \& McKee's (2008) model, a surface density of $1 \mathrm{~g} \mathrm{~cm}^{-2}$ allows the gravity of the core to overcome the radiation pressure of the new-born stars, in order to keep the accretion going and form massive stars inside. If the surface density of a dense clump is high, one may also expect that the star formation rate indicator $L_{\mathrm{IR}}$ and the indicator of star formation efficiency $\left(L_{\mathrm{IR}} / \mathrm{M}_{\mathrm{Vir}}\right)$ are also high.

We check for any correlations between the surface density and $M_{\mathrm{Vir}}, L_{\mathrm{IR}}, L_{\mathrm{IR}} / M_{\mathrm{Vir}}$, and line luminosity $L^{\prime}$ for the four dense gas tracers (HCN 1-0, HCN 3-2, CS 2-1, CS 7-6) in Figures 31-34. There are no obvious correlations found between the surface density and these parameters for dense clumps, except for the very lower end of the luminosity for a few lowmass cores, whose surface density is lower than massive clumps. In particular, $L_{\mathrm{IR}}$ ranges over four orders of magnitude within a total variation of $\Sigma_{\mathrm{HCN} 3-2}$ of about a factor of 10 for dense clumps. We see no evidence for a sharp threshold in surface density for massive dense clumps.

Of course, the threshold is probably not exact, and our values have substantial uncertainty, so generally speaking, these massive clumps are in the right range to form massive stars. With density gradients, most of the dense clumps in our sample will reach the critical value of $1 \mathrm{~g} \mathrm{~cm}^{-2}$ at some radius. The amount of mass above the threshold might help to determine the mass of the stars that can form. The CS 7-6 line comes closest to being a tracer of surface density of $1 \mathrm{~g} \mathrm{~cm}^{-2}$. The mass inside the CS 7-6 contour is on average about 0.25 of the mass inside the CS $2-1$ contour and has a mean value of $1300 M_{\odot}$. Thus, these clumps on average have plenty of mass at high enough surface densities to form clusters of massive stars.

The volume density of a cloud is often taken to control the star formation rate of the cloud. We calculated the mean volume density $\left(n \equiv n\left(\mathrm{H}_{2}\right)+n(\mathrm{He})+\cdots=\rho / \mu \mathrm{m}_{\mathrm{H}}\right.$, with $\mu=2.37$ (Kauffmann et al. 2008) within the FWHM size of the clumps for each transition, according the following equation:

$$
\bar{n}_{\text {transition }}=\frac{M_{\mathrm{vir}}\left(R_{\text {transition }}\right)}{\frac{4}{3} \pi \mu \mathrm{m}_{\mathrm{H}} R_{\text {transition }}^{3}} \approx 4.08 \frac{\left(M_{\mathrm{vir}} / M_{\odot}\right)}{\left(R_{\text {transition }} / 1 \mathrm{pc}\right)^{3}} .
$$

The derived volume densities for each transition are tabulated in Table 11.

The logarithmic averages over all clumps of the mean volume density $\langle\log \bar{n}\rangle$ are $4.1 \pm 0.1,4.3 \pm 0.1,5.1 \pm 0.1$, and $5.4 \pm 0.1$ for HCN 1-0, CS 2-1, HCN 3-2, and CS 7-6, respectively. The uncertainties are the standard deviation of the mean. In Plume et al. (1997), the volume density of a similar sample of clumps was derived by fitting multiple $\mathrm{CS}$ and $\mathrm{C}^{34} \mathrm{~S}$ spectral lines toward the center position with an LVG model for the excitation. The logarithmic average over the sample was $\left\langle\log n_{\mathrm{LVG}}\right\rangle=5.9 \pm 0.2$. 


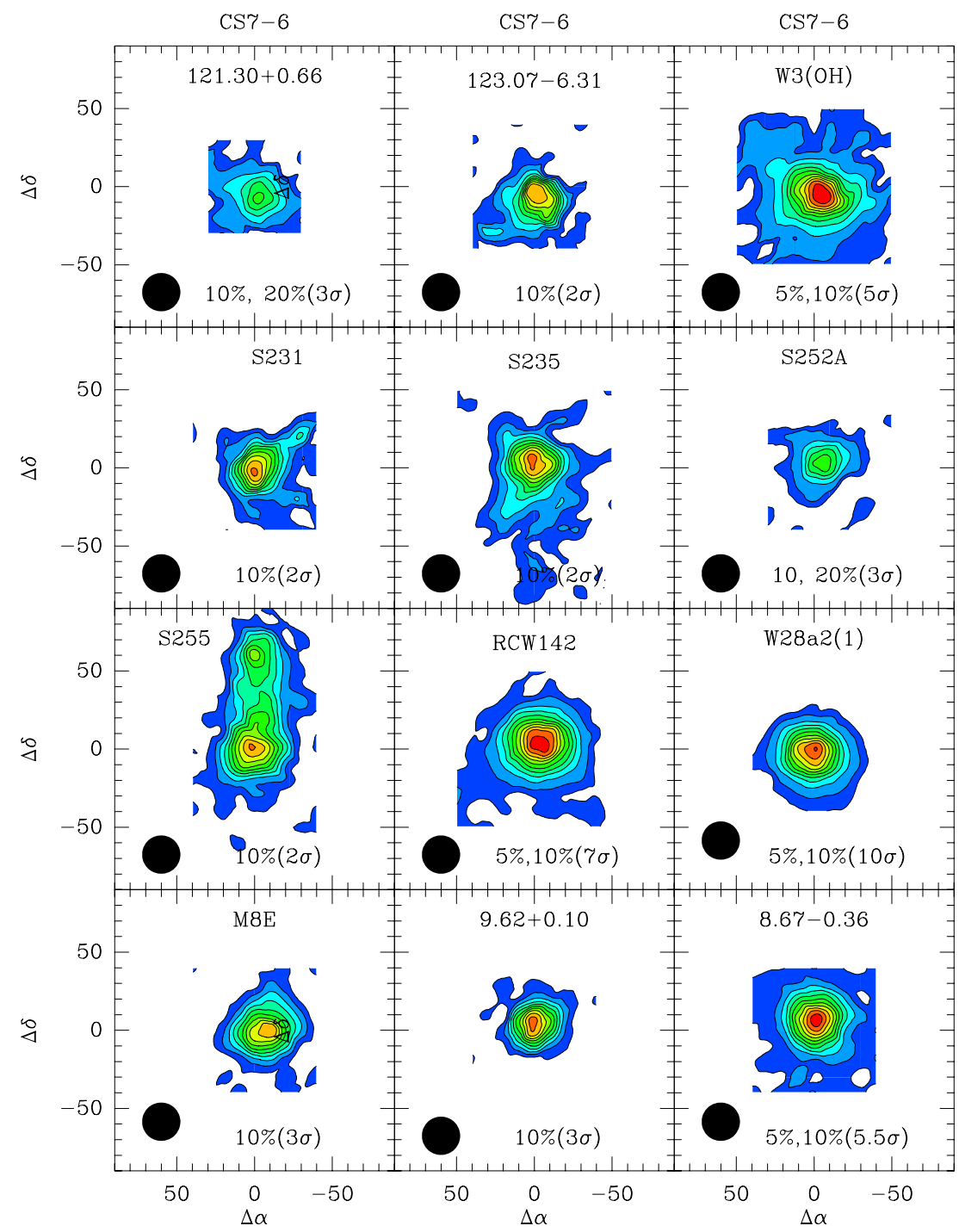

Figure 19. CS 7-6 contour maps of massive clumps. The lowest contour level and increasing step of contours are indicated in the plot. The beam size is shown at the lower left of each map.

(A color version of this figure is available in the online journal.)

The excitation densities $\left(n_{\mathrm{LVG}}\right)$ derived from the LVG model (Plume et al. 1997) are larger than mean densities $(\bar{n})$ derived from the mass and size for two reasons. The value of $\bar{n}$ is an average over all the gas within the FWHM contour traced by a certain tracer. Even the CS 7-6 maps cover more than one to a few beam sizes. The density derived from single point spectrum with an LVG model was calculated from one beam measurement at the peak. Since the clumps are centrally condensed, $\bar{n}$ will be less than $n$ from the LVG model. In addition, the excitation requirements of the hardest-to-excite transition in the LVG fit push the fit to the higher densities in the region. The fact that the values of $\bar{n}$ from the highest excitation lines are closest to the values from the LVG models is a reflection of this point. These points were discussed in detail by Plume et al. (1997).

If the star formation rate is proportional to the amount of gas and inversely proportional to the free-fall time, a simple prediction is that $\dot{M}_{\star} \propto \bar{n} / t_{f f} \propto \bar{n}^{1.5}$. This idea has been used (e.g., Krumholz et al. 2007; Narayanan et al. 2008) to explain the basis of the observed Kennicutt-Schmidt relations for star formation in galaxies. Is the star formation rate proportional to the mean density to the power of 1.5 , as theorists have suggested?
We plot the mean volume density of each tracer versus infrared luminosity in Figure 35. Linear least-square fits with uncertainty give the following results:

$$
\text { Least squares : } \begin{aligned}
\log \left(L_{\mathrm{IR}}\right)= & -3.42( \pm 0.28) \times \log \left(\bar{n}\left(R_{\mathrm{HCN} 1-0}\right)\right) \\
& +20.11( \pm 1.26), r=-0.55
\end{aligned}
$$

Robust fit : $\log \left(L_{\mathrm{IR}}\right)=-1.02 \times \log \left(\bar{n}\left(R_{\mathrm{HCN} 1-0}\right)\right)+8.78$

Least squares : $\log \left(L_{\mathrm{IR}}\right)=-5.28( \pm 0.73) \times \log \left(\bar{n}\left(R_{\mathrm{HCN} 3-2}\right)\right)$ $+33.35( \pm 3.99), r=-0.57$

Robust fit $: \log \left(L_{\mathrm{IR}}\right)=-1.2 \times \log \left(\bar{n}\left(R_{\mathrm{HCN} 3-2}\right)\right)+10.96$

Least squares : $\log \left(L_{\mathrm{IR}}\right)=-3.46( \pm 0.25) \times \log \left(\bar{n}\left(R_{\mathrm{CS} 2-1}\right)\right)$ $+20.46( \pm 1.14), r=-0.49$

Robust fit $: \log \left(L_{\mathrm{IR}}\right)=-0.90 \times \log \left(\bar{n}\left(R_{\mathrm{CS} 2-1}\right)\right)+8.35$ 


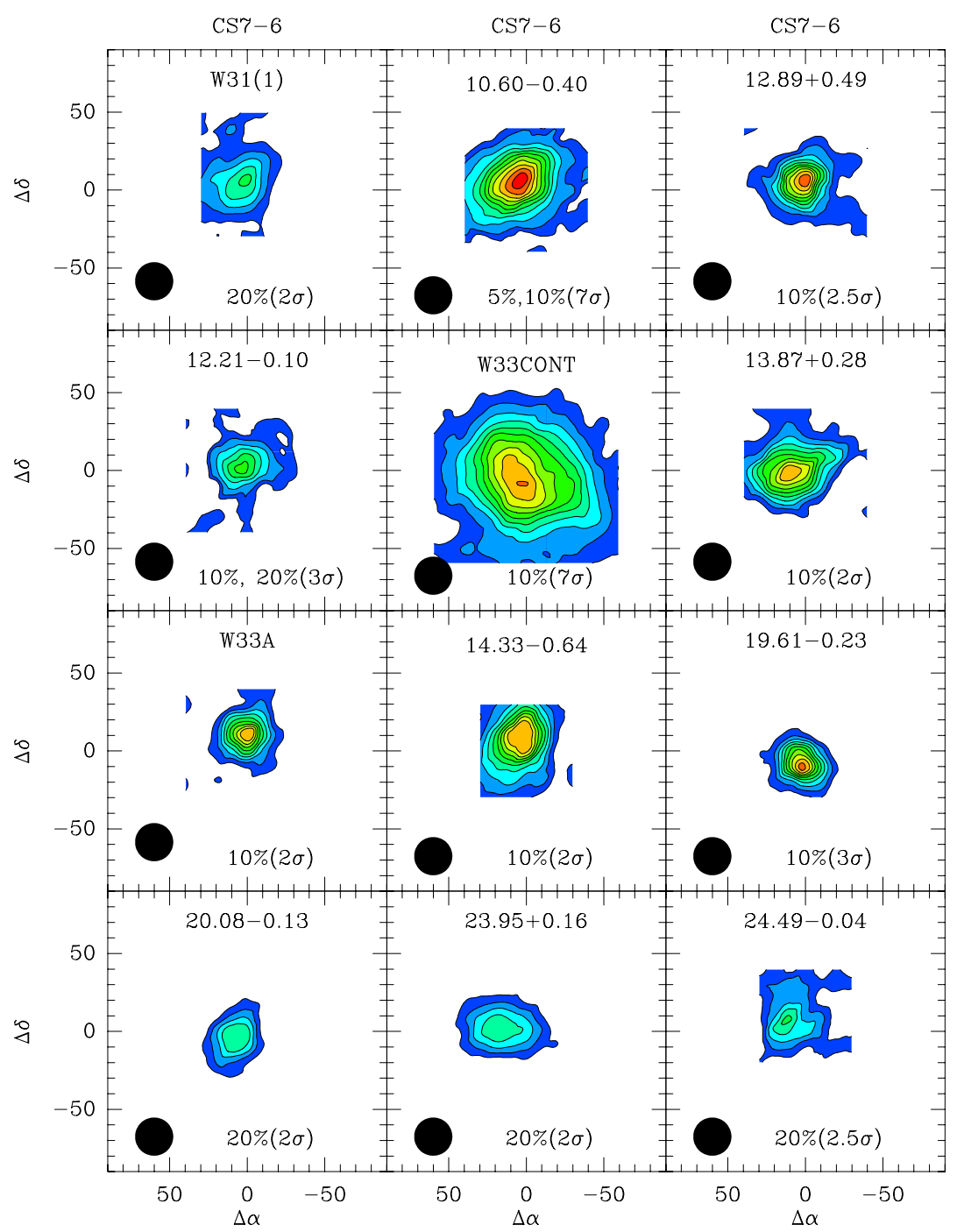

Figure 20. Same as Figure 19.

(A color version of this figure is available in the online journal.)

$$
\text { Least squares : } \begin{aligned}
\log \left(L_{\mathrm{IR}}\right)= & -1.18( \pm 0.51) \times \log \left(\bar{n}\left(R_{\mathrm{CS} 7-6}\right)\right) \\
& +11.78( \pm 0.30), r=-0.37
\end{aligned}
$$

Robust fit : $\log \left(L_{\mathrm{IR}}\right)=-0.70 \times \log \left(\bar{n}\left(R_{\mathrm{CS} 7-6}\right)\right)+8.90$.

Far from the prediction that $\bar{n}$ and $L_{\mathrm{IR}}$ are positively correlated, in general, $L_{\mathrm{IR}}$ decreases with increasing $\bar{n}$. (There may be a hint of positive correlation for the few low-luminosity cores with adequate data.) Interestingly, all the fits from robust estimation have slopes near -1 with mean value over all tracers of $-0.96 \pm 0.21$. Since most massive clumps are large, they have lower mean densities; this fact probably explains the negative correlation if luminosity is just proportional to mass above a threshold density. A similar effect is seen in the plot of $L_{\mathrm{mol}}^{\prime}$ versus $\bar{n}$ (Figure 36). This result is also plausible because $L_{\text {mol }}^{\prime}$ is almost linearly correlated with $L_{\mathrm{IR}}$ (see Section 5.2).

We can also plot $L_{\mathrm{IR}}$ versus $n_{\mathrm{LVG}}$, which traces the denser part of a clump with a density gradient. The plot of $L_{\mathrm{IR}}$ versus $n_{\mathrm{LVG}}$ (Figure 37) reveals a very weak positive correlation with $L_{\mathrm{IR}}$. The linear least-square fit with uncertainty gives

$$
\begin{aligned}
\log \left(L_{\mathrm{IR}}\right)= & 15.7( \pm 10.1) \times \log (n(\mathrm{LVG})) \\
& -87.9( \pm 119.3), r=0.18 .
\end{aligned}
$$

We see no sign of $L_{\mathrm{IR}} \propto n^{1.5}$ for these massive dense clumps, regardless of how density is defined.

We also plotted the correlation between the star formation efficiency (indicated by the infrared luminosity derived by virial mass) and the virial mass for different tracers (Figure 38). It shows no trend between them.

Comparing the correlations between $L_{\mathrm{IR}}$ and virial mass, surface density, and volume density for dense clumps, we found that only the virial mass has a strong correlation with $L_{\mathrm{IR}}$; the correlations between $L_{\mathrm{IR}}$ and surface density or mean volume density are weak. It suggests that the most relevant parameter for the star formation rate in a dense clump is how much gas above a threshold density it contains. The surface density or mean density within the clump is not the controlling factors.

\subsection{Do Line Luminosity Ratios Correlate with Other Properties?}

Table 8 gives the mean, median, and standard deviation of the ratios between the various lines. The luminosity ratio of high- $J$ to low- $J$ transitions could reflect the ratio of very dense gas to less dense gas in a clump. They could also depend on the infrared luminosity because a higher luminosity will result in a greater fraction of the gas being warm enough to emit in the 


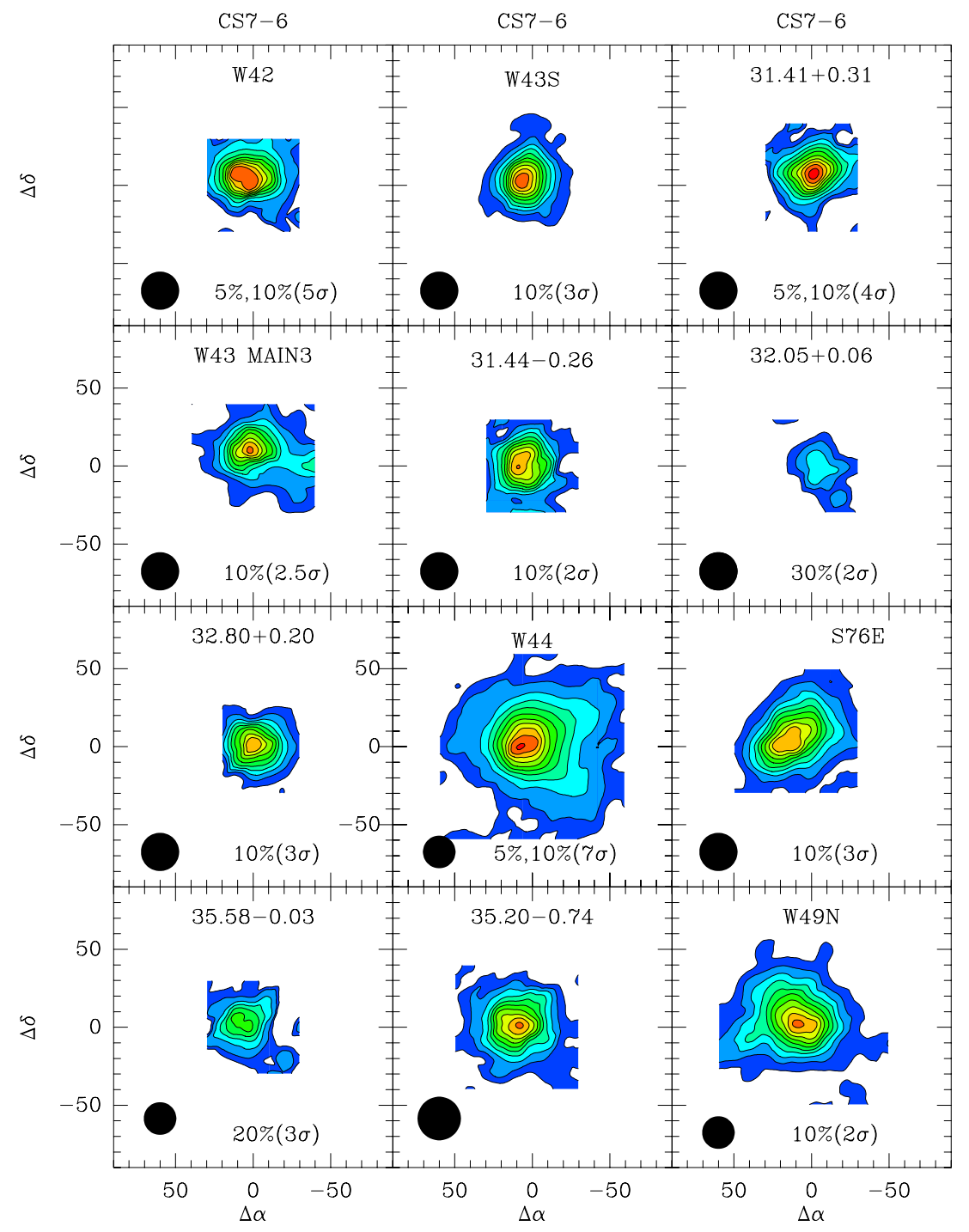

Figure 21. Same as Figure 19.

(A color version of this figure is available in the online journal.)

higher- $J$ lines. We have tested this latter possibility by plotting line luminosity ratios versus infrared luminosity $L_{\mathrm{IR}}$. Figure 39 indicates that only the ratio $\frac{L_{\mathrm{CS} 7-6}^{\prime}}{L_{\mathrm{CSS}-4}^{\prime}}$ shows a weak correlation with $L_{\mathrm{IR}}$. It can be fitted by a linear correlation:

$$
\log \left(\frac{L_{\mathrm{CS} 7-6}^{\prime}}{L_{\mathrm{CS} 5-4}^{\prime}}\right)=0.18( \pm 0.05) \times \log \left(L_{\mathrm{IR}}\right)-1.11( \pm 0.26) .
$$

The correlation coefficient $r$ is 0.49 . Furthermore, the spread in values of the ratio is modest for most ratios (Table 8). The linear fit for other ratios versus $L_{\mathrm{IR}}$ show weak or no correlations:

$$
\begin{aligned}
\log \left(\frac{L_{\mathrm{CS} 7-6}^{\prime}}{L_{\mathrm{CS} 2-1}^{\prime}}\right)= & 0.09( \pm 0.10) \times \log \left(L_{\mathrm{IR}}\right) \\
& -1.28( \pm 0.53), r=0.17 \\
\log \left(\frac{L_{\mathrm{CS} 5-4}^{\prime}}{L_{\mathrm{CS} 2-1}^{\prime}}\right)= & -0.01( \pm 0.06) \times \log \left(L_{\mathrm{IR}}\right) \\
& -0.54( \pm 0.30), r=-0.03
\end{aligned}
$$

$$
\begin{aligned}
\log \left(\frac{L_{\mathrm{HCN} 3-2}^{\prime}}{L_{\mathrm{HCN} 1-0}^{\prime}}\right)= & 0.084( \pm 0.03) \times \log \left(L_{\mathrm{IR}}\right) \\
& -0.10( \pm 0.14), r=0.46 .
\end{aligned}
$$

Thus, with the possible exception of the CS 7-6 line, we find little dependence of the line ratios on luminosity, suggesting that temperature differences are not important for most ratios. In particular, the ratio of $\frac{L_{\mathrm{HCN} 1-0}^{\prime}}{L_{\mathrm{HCN} 3-2}}$ is nearly constant for these clumps, with much less scatter than other ratios. These ratios are primarily sensitive to the density structure for these massive clumps, and the uniformity of the values reflects the fact that most dense clumps have power-law density distributions (Mueller et al. 2002; Shirley et al. 2003).

We can also correlate the line ratios with the bolometric temperature $\left(T_{\mathrm{bol}}\right)$, which is the temperature of a blackbody with the same mean frequency as the actual SED. In low-mass cores, $T_{\mathrm{bol}}$ is related to the evolutionary status of a core (Chen et al. 1995), with lower $T_{\text {bol }}$ corresponding to earlier stages. In these sources, it might also reflect the relative amounts of gas at higher and lower temperatures. The plots in Figure 40 show little correlation between line ratios and $T_{\mathrm{bol}}$. The linear least-square 


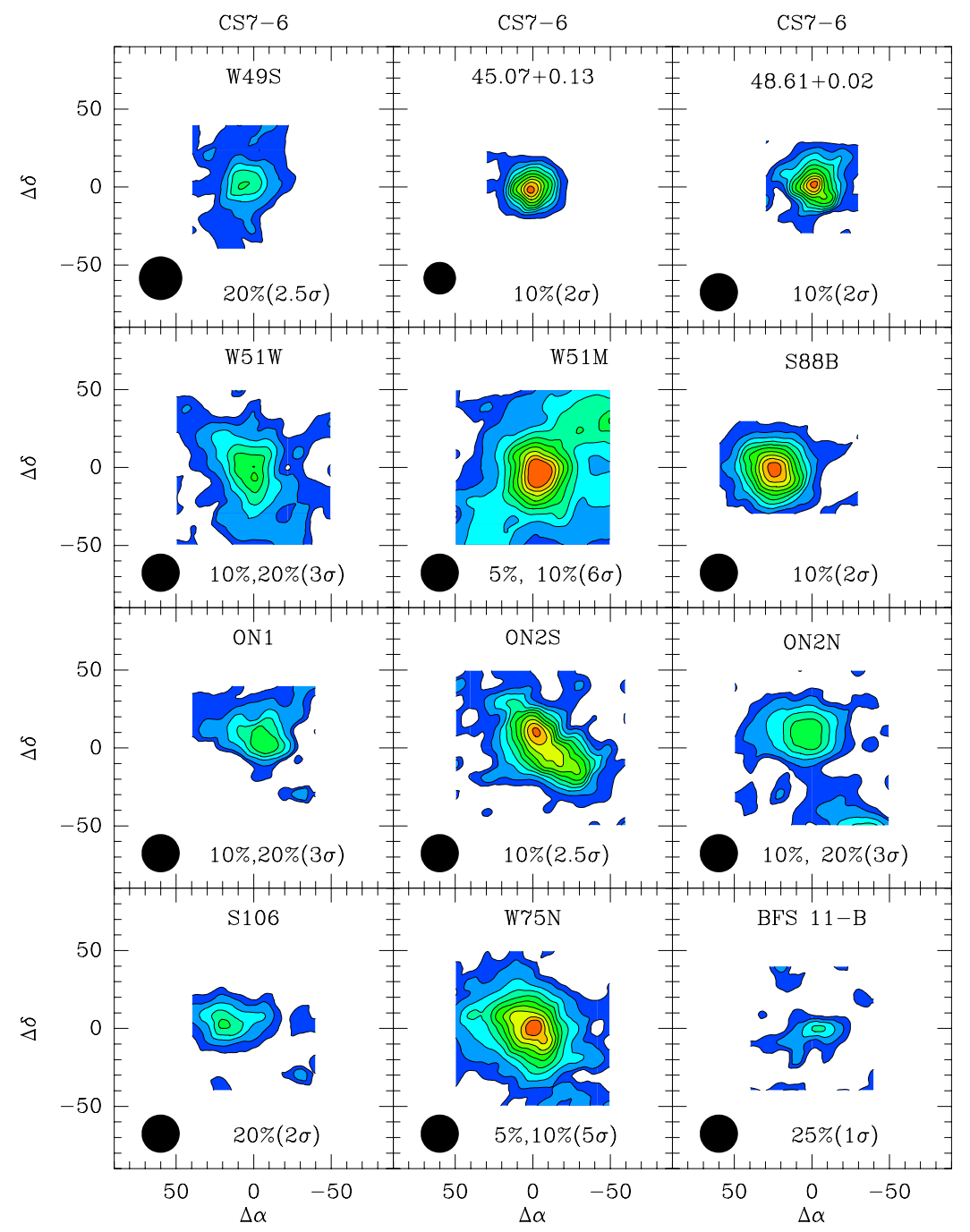

Figure 22. Same as Figure 19.

(A color version of this figure is available in the online journal.)

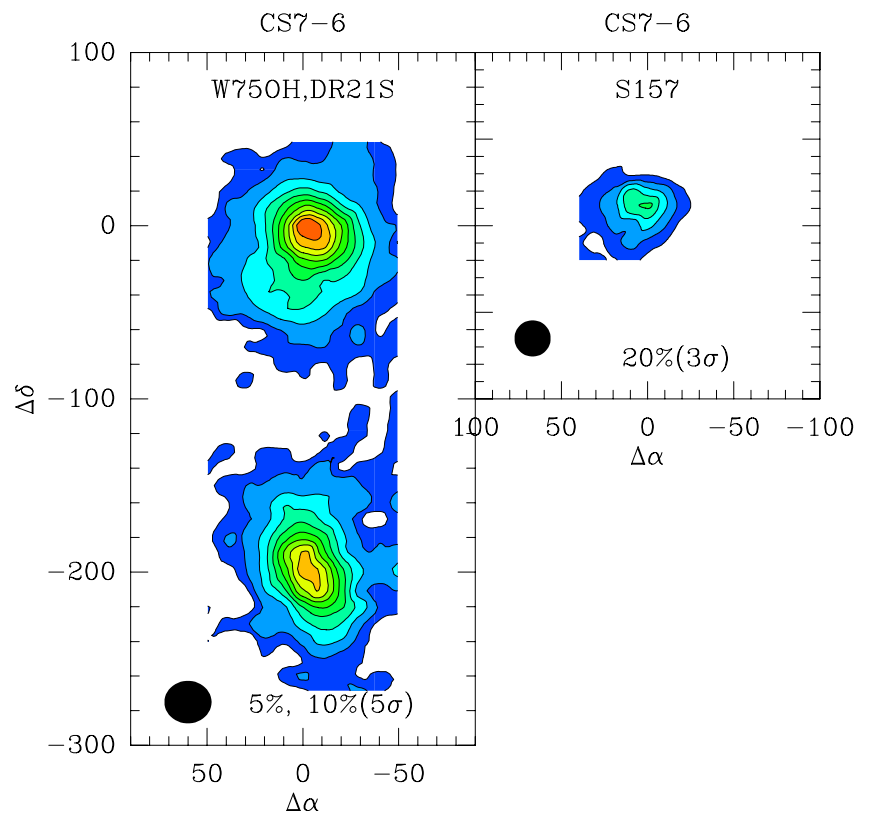

Figure 23. Same as Figure 19.

(A color version of this figure is available in the online journal.) fits to these correlations give:

$$
\begin{aligned}
& \log \left(\frac{L_{\mathrm{CS} 7-6}^{\prime}}{L_{\mathrm{CS} 5-4}^{\prime}}\right)= 0.005( \pm 0.003) \times T_{\mathrm{bol}} \\
&-0.67( \pm 0.22), r=0.32 \\
& \log \left(\frac{L_{\mathrm{CS} 7-6}^{\prime}}{L_{\mathrm{CS} 2-1}^{\prime}}\right)=-0.005( \pm 0.004) \times T_{\mathrm{bol}} \\
&-0.50( \pm 0.32) . r=-0.22 . \\
& \log \left(\frac{L_{\mathrm{CS} 5-4}^{\prime}}{\left.L_{\mathrm{CS} 2-1}^{\prime}\right)=}-0.005( \pm 0.003) \times T_{\mathrm{bol}}\right. \\
&-0.25( \pm 0.24) . r=-0.27 . \\
& \log \left(\frac{L_{\mathrm{HCN} 3-2}^{\prime}}{L_{\mathrm{HCN} 1-0}^{\prime}}\right)= 0.0006( \pm 0.002) \times T_{\mathrm{bol}} \\
&-0.65( \pm 0.20) . r=0.05 .
\end{aligned}
$$

This result suggests that the fraction of gas in various density regimes does not depend strongly on evolutionary stage, at least for those stages probed by our sample. 


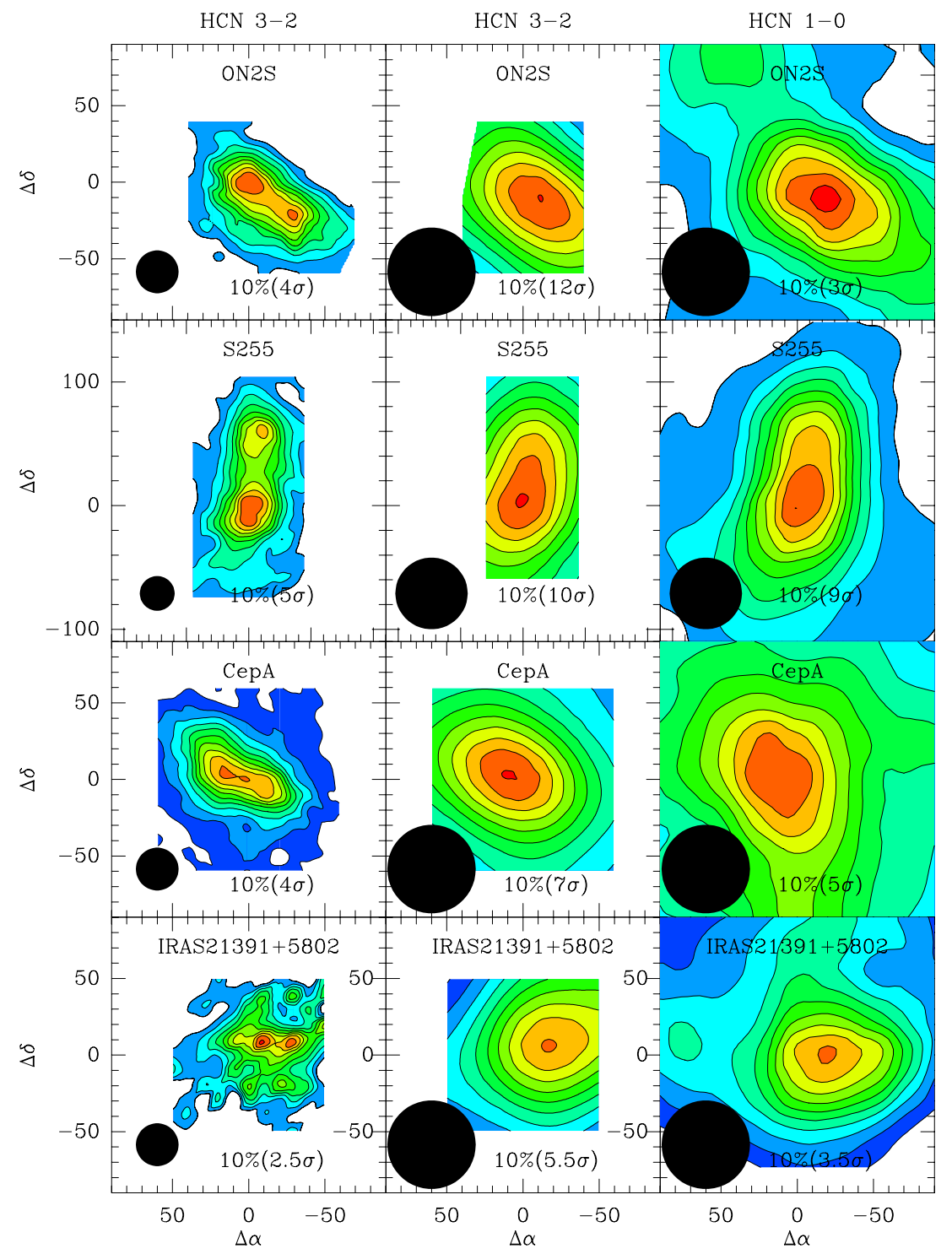

Figure 24. Plots to show the effect when we smooth the HCN 3-2 maps (left panels) of four clumps with more than one peaks, to the resolution of HCN 1-0 data (middle panels), comparing to the real $\mathrm{HCN} 1-0$ maps (right panels).

(A color version of this figure is available in the online journal.)

Do the line ratios correlate with $L_{\mathrm{IR}} / M_{\mathrm{vir}}$, which is a star formation efficiency indicator, if $L_{\mathrm{IR}}$ is a tracing star formation rate? The plots in Figure 41 show no clear trends. These results suggest that all of these tracers are probing gas that is dense enough to be involved in massive star formation. The lower- $J$ lines simply trace a larger region of somewhat lower density and thus have larger map sizes and luminosities.

Another approach to an evolutionary sequence for massive star-forming regions is based on the evolution of $\mathrm{H}$ II regions. In this picture, a clump would evolve from having no radio continuum (NoRC), to an ultracompact H II region stage (UCHII), to a Compact $\mathrm{H}_{\text {II }}$ region stage, and finally to a complete $\mathrm{H}_{\text {II }}$ region stage. In the paper of Shirley et al. (2003), an extensive literature search using the SIMBAD database was performed to find $\mathrm{H}$ II regions associated with these dense clumps. Using the taxonomy of Kurtz (2002), H II regions are classified as UCHII if the diameter of $2 \mathrm{~cm}$ radio continuum emission is $\leqslant 0.1 \mathrm{pc}$, $\mathrm{CHII}$ is the diameter is $\leqslant 0.5 \mathrm{pc}$, and an extended $\mathrm{H}$ II region if the diameter is greater than $0.5 \mathrm{pc}$ or clearly associated with a classical $\mathrm{H}$ II region. The classification of the evolutionary states of these dense clumps refers to Table 1 of Shirley et al. (2003). If we classify the massive clumps in the sample according to this scheme, do we see any trends? We plot the line ratio of $\frac{L^{\prime}\left(\mathrm{CS}_{7}-6\right)}{L^{\prime}(\mathrm{CS} 5-4)}$ (the ratio of two tracers that form a well-matched sample in our survey, so we have enough points to make the statistics) versus star formation efficiency in Figure 42 with different symbols for each $\mathrm{H}$ II region status. No obvious differences emerge in the plot. The mean, median, and standard deviation of this ratio for each evolutionary stage are tabulated in Table 12 . We see no obvious trend for the ratio of $\frac{L^{\prime}\left(\mathrm{CS}_{7}-6\right)}{L^{\prime}\left(\mathrm{CS}_{5}-4\right)}$ along the evolutionary sequence, except that the mean and median ratio is lower for sources with no radio continuum emission than other three categories, indicating either that the dense gas ratio is lower or the temperatures are lower in this stage.

\subsection{Dynamical Properties}

The dynamical state of molecular clumps is an important input into star formation theories. As has been discussed many times, the linewidths of massive clumps are much wider than expected 
Table 6

The FWHM Sizes of Maps

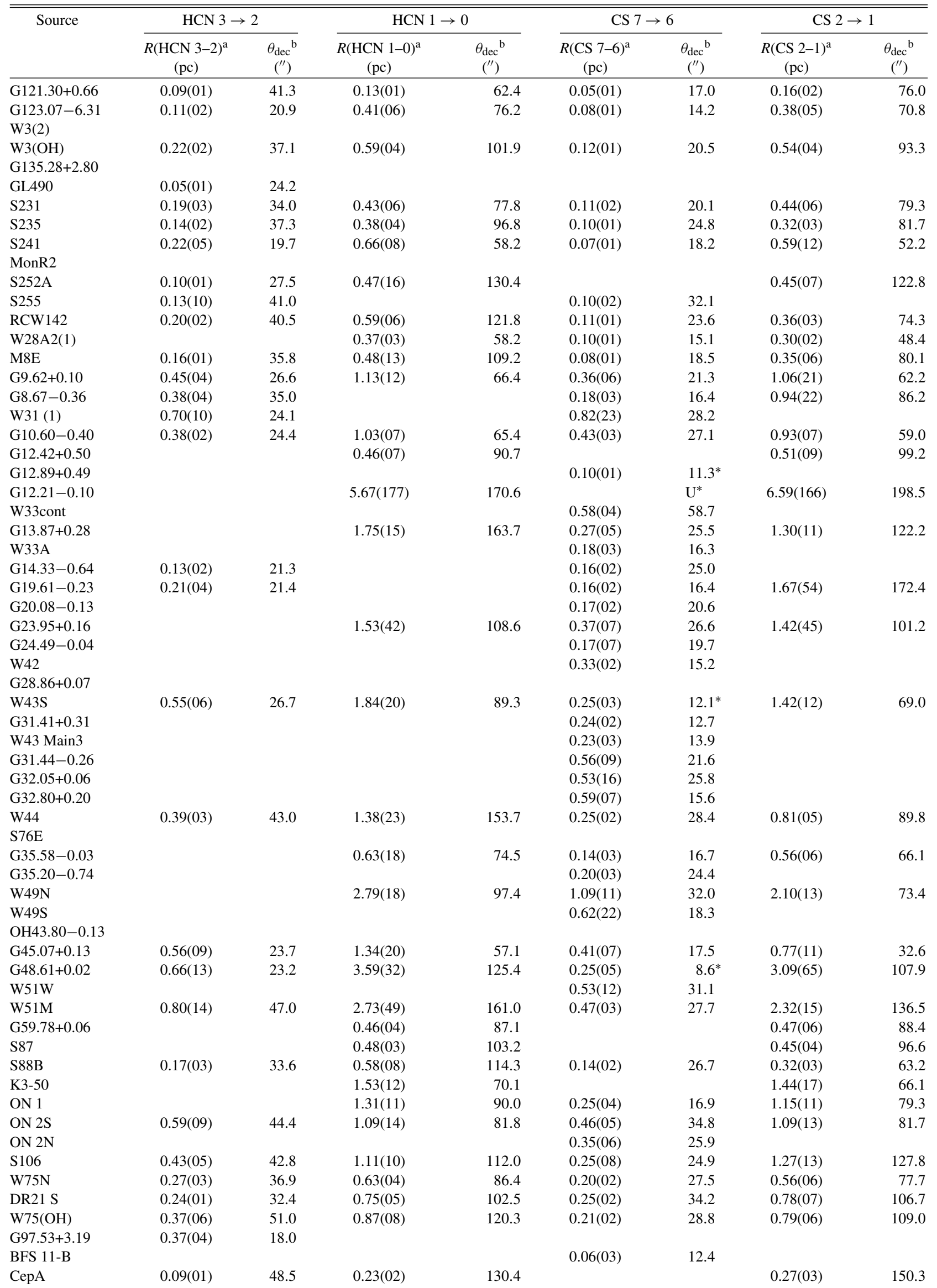


Table 6

(Continued)

\begin{tabular}{|c|c|c|c|c|c|c|c|c|}
\hline \multirow[t]{2}{*}{ Source } & \multicolumn{2}{|c|}{$\mathrm{HCN} 3 \rightarrow 2$} & \multicolumn{2}{|c|}{$\mathrm{HCN} 1 \rightarrow 0$} & \multicolumn{2}{|c|}{$\mathrm{CS} 7 \rightarrow 6$} & \multicolumn{2}{|c|}{$\mathrm{CS} 2 \rightarrow 1$} \\
\hline & $\begin{array}{c}R(\mathrm{HCN} 3-2)^{\mathrm{a}} \\
(\mathrm{pc})\end{array}$ & $\begin{array}{c}\theta_{\mathrm{dec}}^{\mathrm{b}} \\
(")\end{array}$ & $\begin{array}{c}R(\mathrm{HCN} 1-0)^{\mathrm{a}} \\
(\mathrm{pc})\end{array}$ & $\begin{array}{c}\theta_{\mathrm{dec}}^{\mathrm{b}} \\
(")\end{array}$ & $\begin{array}{c}R(\mathrm{CS} 7-6)^{\mathrm{a}} \\
(\mathrm{pc})\end{array}$ & $\begin{array}{c}\theta_{\mathrm{dec}^{\mathrm{b}}} \\
\left({ }^{\prime \prime}\right)\end{array}$ & $\begin{array}{c}R(\mathrm{CS} 2-1)^{\mathrm{a}} \\
(\mathrm{pc})\end{array}$ & $\begin{array}{c}\theta_{\mathrm{dec}^{\mathrm{b}}} \\
\left({ }^{\prime \prime}\right)\end{array}$ \\
\hline S158 & $0.46(05)$ & 68.3 & $0.85(06)$ & 125.9 & & & $0.77(06)$ & 113.6 \\
\hline S158A & $0.37(03)$ & 55.2 & & & & & & \\
\hline NGC7538-IRS9 & $0.42(10)$ & 62.3 & & & & & & \\
\hline S157 & $0.19(04)$ & 30.9 & $0.45(06)$ & 74.5 & $0.15(04)$ & 24.2 & $0.43(08)$ & 71.7 \\
\hline IRAS19094+0944 & & & $1.48(45)$ & 100.0 & & & $1.24(66)$ & 84.0 \\
\hline IRAS20050+2720 & $0.08(02)$ & 43.1 & $0.23(06)$ & 127.5 & & & $0.22(11)$ & 123.8 \\
\hline IRAS20106+3545 & & & $0.24(06)$ & 60.3 & & & $0.27(07)$ & 67.1 \\
\hline IRAS20126+4104 & & & $0.48(04)$ & 47.3 & & & $0.49(08)$ & 48.4 \\
\hline IRAS20188+3928 & $0.03(01)$ & 35.7 & $0.07(01)$ & 98.1 & & & $0.08(01)$ & 105.5 \\
\hline IRAS20216+4107 & $0.05(02)$ & $11.6^{*}$ & $0.26(03)$ & 62.4 & & & $0.20(04)$ & 49.7 \\
\hline IRAS $20220+3728$ & & & $1.40(28)$ & 128.4 & & & $1.41(50)$ & 129.3 \\
\hline IRAS20333+4102 & $0.28(08)$ & 71.1 & $0.42(03)$ & 108.6 & & & $0.40(04)$ & 103.0 \\
\hline IRAS21391+5802 & $0.06(01)$ & 34.5 & $0.14(02)$ & 77.0 & & & $0.14(01)$ & 74.3 \\
\hline IRAS $22172+5549$ & & & $0.36(06)$ & 51.2 & & & $0.40(10)$ & 56.8 \\
\hline IRAS22198+6336 & & & $0.31(07)$ & 100.0 & & & $0.33(07)$ & 106.7 \\
\hline IRAS23011+6126 & $0.03(01)$ & 17.3 & $0.17(03)$ & 98.7 & & & $0.17(04)$ & 94.7 \\
\hline IRAS23385+6053 & & & $1.13(17)$ & 67.3 & & & $1.06(17)$ & 63.2 \\
\hline IRAS03282+3035 & $0.02(01)$ & 46.5 & $0.10(03)$ & 187.0 & & & $0.09(03)$ & 173.8 \\
\hline L483 & $0.02(01)$ & 32.2 & & & & & & \\
\hline L1251B & & & $0.07(01)$ & 92.1 & & & $0.09(02)$ & 127.3 \\
\hline Mean $^{c}$ & $0.32(20)$ & $35(12)$ & $1.13(109)$ & $100(31)$ & $0.28(21)$ & $22(8)$ & $1.03(112)$ & $90(31)$ \\
\hline Median $^{c}$ & 0.26 & 35 & 0.71 & 97 & 0.23 & 21 & 0.77 & 81 \\
\hline
\end{tabular}

Notes.

${ }^{a}$ Numbers in the parentheses are uncertainties of FWHM size in the unit of $0.01 \mathrm{pc}$.

${ }^{b}$ The angular FWHM size of the core deconvolved from the beam size.

${ }^{\mathrm{c}}$ Statistics do not include sources below the horizontal line in this table.

* These sources are either unresolved, or derived sizes are less than half of the beam size for this transition. This may cause the underestimation of the line luminosity. We will ignore these luminosities in later statistics.
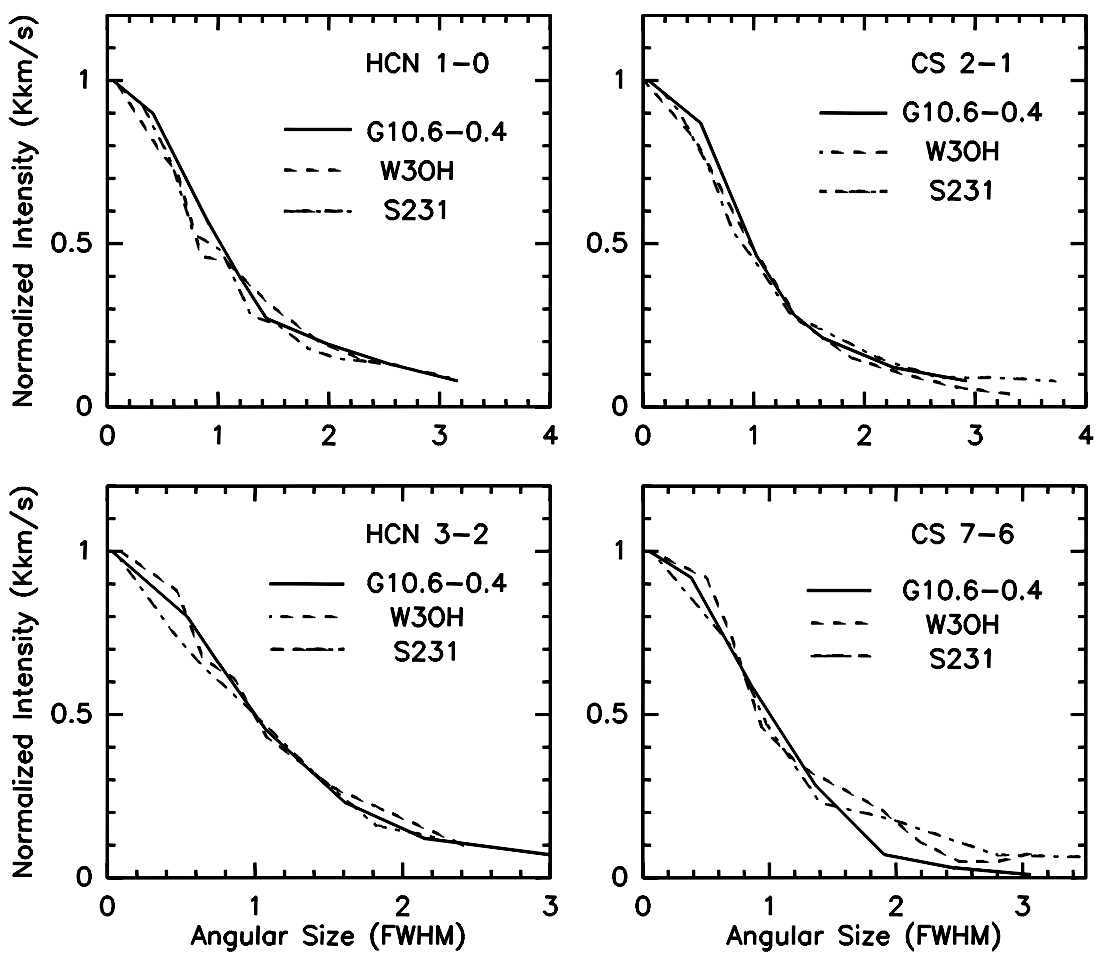

Figure 25. One-dimensional radial profiles of some dense clumps. The intensities have been normalized to the peak values, and the angular radii (convolved with beam sizes) are in units of the FWHM radius of each source. 

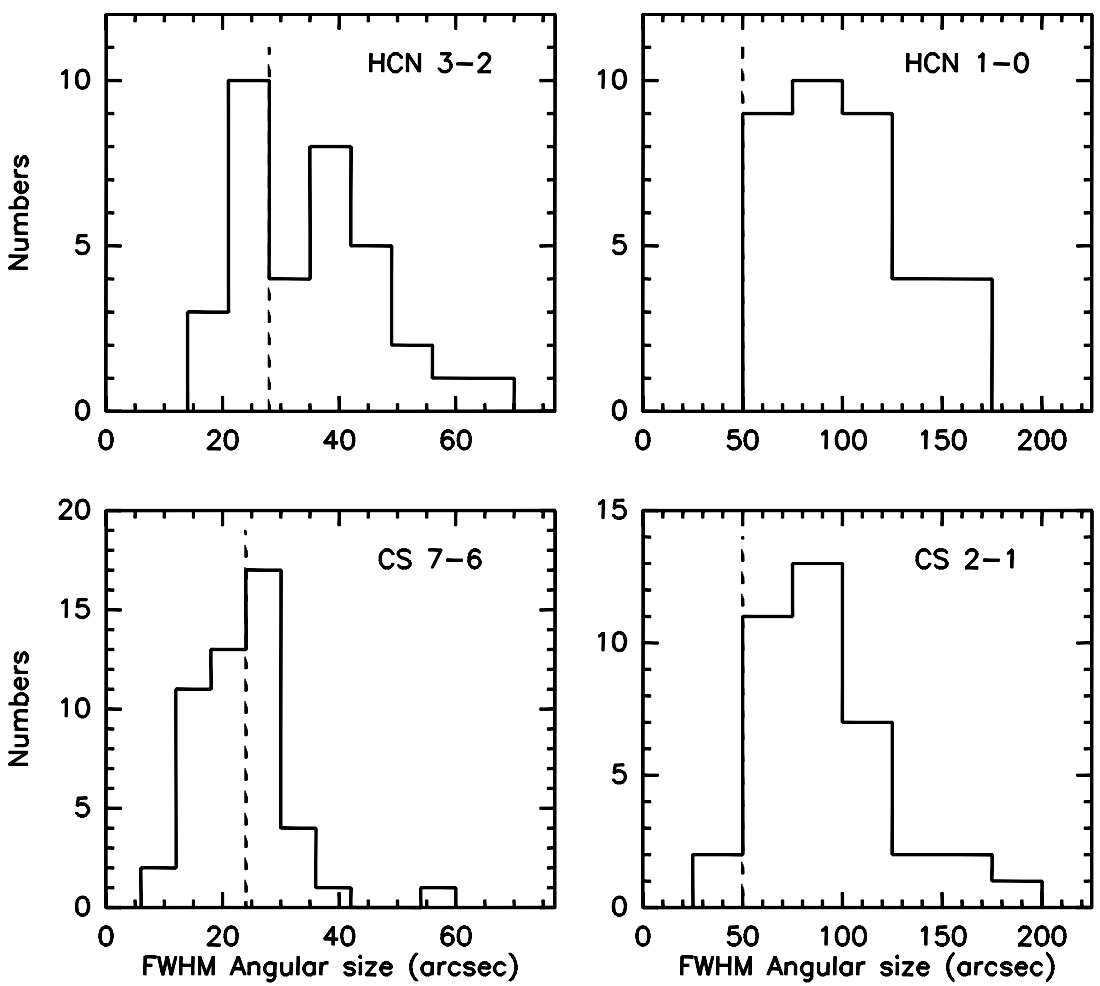

Figure 26. Histograms of the deconvolved angular size of massive dense clumps in different transitions. The vertical dashed line in each panel indicates the beam size of each transitions.
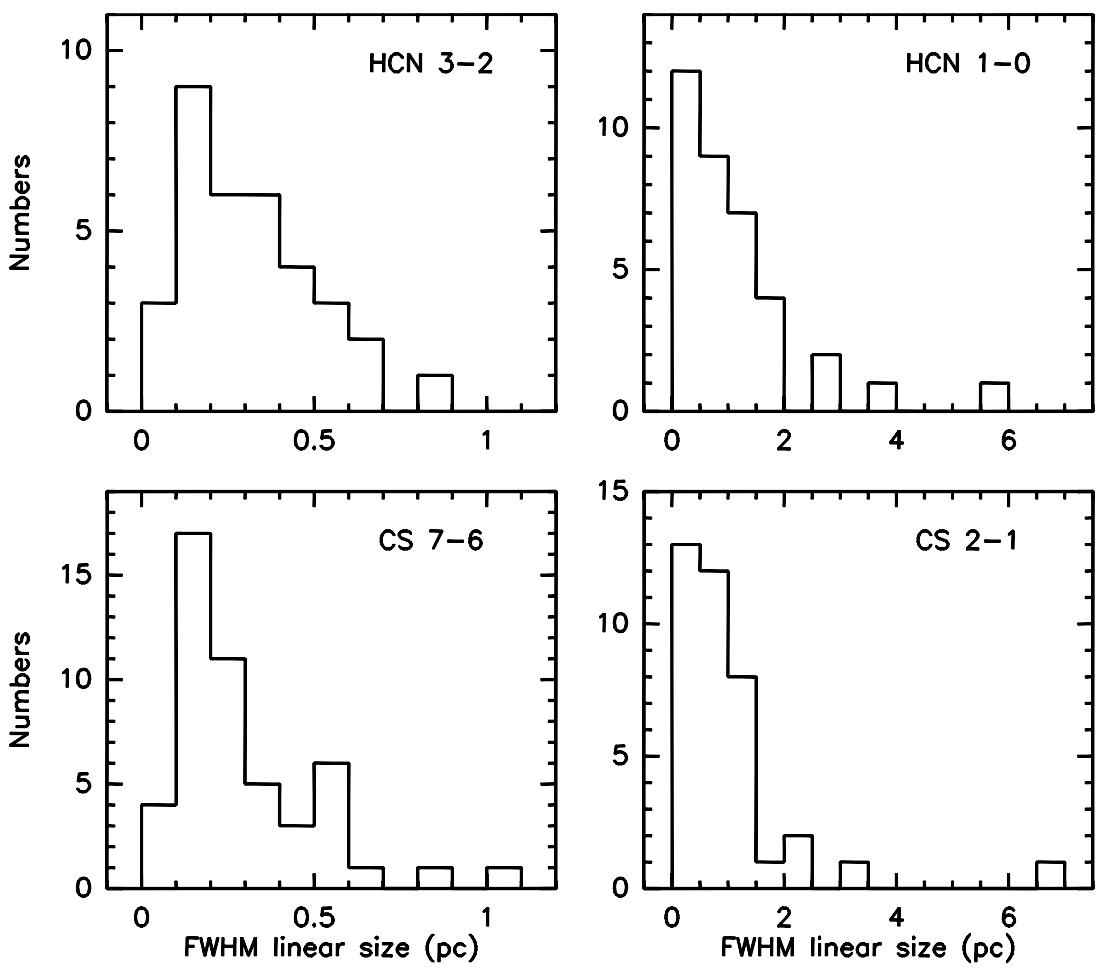

Figure 27. Histograms of the deconvolved linear size of massive dense clumps in different transitions.

for thermal broadening, and some form of turbulence is clearly important. For example, the characteristic temperature in these dense clumps is less than about $100 \mathrm{~K}$. At this temperature, the sound speed is $0.7 \mathrm{~km} \mathrm{~s}^{-1}$. The mean FWHM linewidth from an optically thin tracer $\mathrm{C}^{34} \mathrm{~S} 5-4$ for these massive sources is 4.7 $\mathrm{km} \mathrm{s}^{-1}$. This gives a Mach number of 6.7. Thermal broadening can only account for a small fraction of this broad linewidth.
The turbulence is clearly supersonic and seems to increase on smaller scales, as discussed above.

Evidence for infalling gas can be seen in the form of line profiles skewed to the blue in optically thick lines compared to optically thin lines. Evidence for a significant excess of blueskewed profiles over red-skewed profiles was seen in low-mass Class 0 (Gregersen et al. 1997) and Class I sources (Gregersen 
Table 7

Luminosities of Massive Clumps ${ }^{\mathrm{a}}$

\begin{tabular}{|c|c|c|c|c|c|c|}
\hline Source & $\begin{array}{c}\log L_{\mathrm{IR}} \\
\left(L_{\odot}\right)\end{array}$ & $\begin{array}{c}L^{\prime}(\mathrm{HCN} \mathrm{1-0)} \\
\left(\mathrm{K} \mathrm{km} \mathrm{s}^{-1} \mathrm{pc}^{2}\right) \\
\end{array}$ & $\begin{array}{c}L^{\prime}(\mathrm{HCN} \mathrm{3-2)} \\
\left(\mathrm{K} \mathrm{km} \mathrm{s}^{-1} \mathrm{pc}^{2}\right) \\
\end{array}$ & $\begin{array}{c}L^{\prime}(\mathrm{CS} 7-6) \\
\left(\mathrm{K} \mathrm{km} \mathrm{s}^{-1} \mathrm{pc}^{2}\right) \\
\end{array}$ & 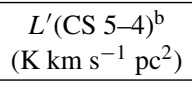 & $\begin{array}{c}L^{\prime}(\mathrm{CS} \mathrm{2-1)} \\
\left(\mathrm{K} \mathrm{km} \mathrm{s}^{-1} \mathrm{pc}^{2}\right) \\
\end{array}$ \\
\hline G121.30+0.66 & 2.85 & $0.53(12)$ & $0.09(17)$ & $-0.29(26)$ & $0.18(13)$ & $0.45(15)$ \\
\hline G123.07-6.31 & 3.76 & $1.36(16)$ & $0.60(15)$ & $0.15(18)$ & $0.63(14)$ & $1.16(14)$ \\
\hline $\mathrm{W} 3(\mathrm{OH})$ & 5.09 & $1.96(06)$ & $1.26(08)$ & $1.07(08)$ & $1.24(10)$ & $1.81(09)$ \\
\hline GL490 & 3.37 & & $-0.41(17)$ & & & \\
\hline S231 & 4.03 & $1.49(16)$ & $0.96(17)$ & $0.29(17)$ & $0.78(15)$ & $1.37(15)$ \\
\hline S235 & 3.72 & $1.36(12)$ & $0.70(13)$ & $0.31(15)$ & $0.66(12)$ & $1.17(10)$ \\
\hline S241 & 4.14 & $1.49(14)$ & $0.83(27)$ & & $0.66(26)$ & $1.39(22)$ \\
\hline S252A & 4.56 & $1.41(65)$ & $0.35(15)$ & $-0.43(19)$ & $0.14(15)$ & $1.19(23)$ \\
\hline $\mathrm{S} 255$ & 4.20 & & $0.76(11)$ & $0.71(18)$ & & \\
\hline RCW142 & 4.82 & $2.22(11)$ & $1.68(12)$ & $0.97(06)$ & $1.26(10)$ & $1.73(09)$ \\
\hline W28A2(1) & 5.45 & $1.72(09)$ & & $0.87(05)$ & $1.64(10)$ & $1.66(07)$ \\
\hline M8E & 4.26 & $1.38(39)$ & $0.95(08)$ & $-0.01(13)$ & $0.64(11)$ & $1.12(19)$ \\
\hline $9.62+0.19$ & 5.75 & $2.34(11)$ & $2.01(08)$ & $1.87(20)$ & $1.75(10)$ & $2.22(23)$ \\
\hline $8.67-0.36$ & 5.12 & & & $0.84(10)$ & $1.48(11)$ & $2.01(29)$ \\
\hline W31(1) & 6.22 & & $2.57(15)$ & $2.18(35)$ & $2.38(11)$ & \\
\hline $10.60-0.40$ & 6.05 & $2.78(06)$ & $2.31(06)$ & $2.42(06)$ & $2.42(10)$ & $2.54(08)$ \\
\hline $12.42+0.50$ & 4.27 & $1.46(17)$ & & & & $1.35(21)$ \\
\hline $12.89+0.49$ & 4.66 & & & $0.07(12)$ & $1.03(12)$ & \\
\hline $12.21-0.10$ & 5.98 & $3.03(53)$ & & & $2.24(16)$ & $3.16(37)$ \\
\hline W33cont & & & & $3.05(06)$ & $2.54(11)$ & \\
\hline $13.87+0.28$ & 5.29 & $2.89(10)$ & & $1.08(19)$ & $1.15(16)$ & $2.74(09)$ \\
\hline W33A & 5.19 & & & $0.69(19)$ & $1.31(11)$ & \\
\hline $14.33-0.64$ & 4.33 & & $1.16(20)$ & $0.94(17)$ & $1.11(10)$ & \\
\hline $19.61-0.23$ & 5.29 & & $1.54(19)$ & $1.04(12)$ & $1.37(14)$ & $1.74(59)$ \\
\hline $20.08-0.13$ & 4.65 & & & $0.86(14)$ & $0.89(11)$ & \\
\hline $23.95+0.16$ & 5.36 & $2.23(54)$ & & $1.21(22)$ & $1.42(14)$ & $2.06(51)$ \\
\hline $24.49-0.04$ & 4.74 & & & $0.42(69)$ & $0.76(12)$ & \\
\hline W42 & & & & $1.43(07)$ & $1.94(13)$ & \\
\hline $28.86+0.07$ & 5.53 & & & & $1.55(10)$ & \\
\hline W43S & 6.24 & $2.88(12)$ & $2.35(12)$ & $1.19(12)$ & $2.05(12)$ & $2.60(09)$ \\
\hline $31.41+0.31$ & 5.32 & & & $1.17(09)$ & $1.85(11)$ & \\
\hline W 43 Main3 & & & & $1.09(14)$ & $1.87(18)$ & \\
\hline $31.44-0.26$ & 5.78 & & & $1.61(18)$ & $1.84(11)$ & \\
\hline $32.05+0.20$ & 5.02 & & & $1.48(43)$ & $1.50(11)$ & \\
\hline $32.80+0.20$ & & & & $1.93(12)$ & $2.35(12)$ & \\
\hline W44 & 5.48 & $2.17(21)$ & $1.90(09)$ & $1.73(07)$ & $1.96(11)$ & $2.26(06)$ \\
\hline S76E & 4.47 & & & $0.83(12)$ & $1.14(10)$ & \\
\hline $35.58-0.03$ & 4.68 & $1.40(38)$ & & $0.43(19)$ & $0.93(15)$ & $1.41(13)$ \\
\hline $35.20-0.74$ & 4.65 & & & $1.06(18)$ & $1.27(13)$ & \\
\hline W49N & 6.69 & $3.44(06)$ & & $3.29(11)$ & $2.91(11)$ & $3.22(06)$ \\
\hline W49S & & & & $1.81(51)$ & $2.44(19)$ & \\
\hline $43.80-0.13$ & 4.51 & & & & $0.69(11)$ & \\
\hline $45.07+0.13$ & & $2.42(16)$ & $2.08(17)$ & $1.84(18)$ & $2.04(11)$ & $2.24(15)$ \\
\hline $48.61+0.02$ & 6.06 & $3.12(12)$ & $2.07(21)$ & $0.57(22)$ & $1.73(15)$ & $2.69(27)$ \\
\hline W51W & 5.93 & & & $1.89(29)$ & $1.76(23)$ & \\
\hline W51M & 6.60 & $3.40(22)$ & $2.79(20)$ & $2.53(07)$ & $2.64(10)$ & $3.48(07)$ \\
\hline $59.78+0.06$ & 4.10 & $1.53(09)$ & & & $0.58(16)$ & $1.33(14)$ \\
\hline S87 & 4.53 & $1.74(06)$ & & & & $1.41(08)$ \\
\hline S88B & 5.03 & $1.69(16)$ & $0.89(17)$ & $0.47(15)$ & $0.60(12)$ & $1.08(10)$ \\
\hline K3-50 & 6.32 & $2.68(08)$ & & & $1.95(15)$ & $2.32(13)$ \\
\hline ON1 & 5.16 & $2.26(09)$ & & $1.18(20)$ & $1.45(14)$ & $2.14(10)$ \\
\hline ON2S & 5.71 & $2.42(14)$ & $2.21(17)$ & $2.13(13)$ & $1.96(12)$ & $2.33(13)$ \\
\hline ON2N & & & & $1.73(22)$ & $1.67(13)$ & \\
\hline S106 & 5.71 & $2.26(09)$ & $1.64(12)$ & $1.25(44)$ & $1.13(21)$ & $2.02(11)$ \\
\hline W75N & 5.29 & $2.11(07)$ & $1.66(06)$ & $1.50(08)$ & $1.56(11)$ & $1.90(11)$ \\
\hline DR21S & 5.70 & $0.29(06)$ & $0.99(05)$ & $0.81(11)$ & $1.43(12)$ & $2.11(10)$ \\
\hline W75(OH) & 4.70 & $2.39(09)$ & $1.64(19)$ & $1.64(10)$ & $1.54(11)$ & $2.24(08)$ \\
\hline BFS 11-B & 3.88 & & & $-0.40(31)$ & $0.03(39)$ & \\
\hline CEPA & 4.92 & $0.93(12)$ & $0.47(10)$ & & & $0.91(14)$ \\
\hline S158 & 5.29 & $2.40(07)$ & $1.71(12)$ & & $1.63(10)$ & $2.25(08)$ \\
\hline S158A & & & $1.67(08)$ & & & \\
\hline NGC7538-IRS9 & & & $1.31(29)$ & & & \\
\hline S157 & 4.35 & $1.56(14)$ & $0.84(22)$ & $0.60(32)$ & $0.73(13)$ & $1.25(22)$ \\
\hline IRAS19094 & 4.47 & $1.86(45)$ & & & & $1.38(10)$ \\
\hline
\end{tabular}


Table 7

(Continued)

\begin{tabular}{|c|c|c|c|c|c|c|}
\hline Source & $\begin{array}{c}\log L_{\mathrm{IR}} \\
\left(L_{\odot}\right)\end{array}$ & $\begin{array}{c}L^{\prime}(\mathrm{HCN} \mathrm{1-0)} \\
\left(\mathrm{K} \mathrm{km} \mathrm{s}^{-1} \mathrm{pc}^{2}\right)\end{array}$ & $\begin{array}{c}L^{\prime}(\mathrm{HCN} \mathrm{3-2)} \\
\left(\mathrm{K} \mathrm{km} \mathrm{s}^{-1} \mathrm{pc}^{2}\right)\end{array}$ & $\begin{array}{c}L^{\prime}(\mathrm{CS} 7-6) \\
\left(\mathrm{K} \mathrm{km} \mathrm{s}^{-1} \mathrm{pc}^{2}\right)\end{array}$ & 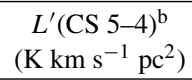 & $\begin{array}{c}L^{\prime}(\mathrm{CS} 2-1) \\
\left(\mathrm{K} \mathrm{km} \mathrm{s}^{-1} \mathrm{pc}^{2}\right)\end{array}$ \\
\hline IRAS20050 & 2.47 & $0.38(39)$ & $-0.27(48)$ & & & $0.25(70)$ \\
\hline IRAS20106 & 3.16 & $0.48(34)$ & & & & $0.23(30)$ \\
\hline IRAS20126 & 4.78 & $1.74(09)$ & & & & $1.35(17)$ \\
\hline IRAS20188 & 2.48 & $-0.15(10)$ & $-0.76(11)$ & & & $-0.24(14)$ \\
\hline IRAS20216 & 3.47 & $0.65(14)$ & & & & $0.24(19)$ \\
\hline IRAS20220 & 4.78 & $2.02(25)$ & & & & $1.94(81)$ \\
\hline IRAS20333 & 3.96 & $1.23(08)$ & $0.54(47)$ & & & $0.77(13)$ \\
\hline IRAS21391 & 2.43 & $0.41(14)$ & $-0.08(26)$ & & & $0.35(11)$ \\
\hline IRAS22172 & 3.56 & $0.99(20)$ & & & & $0.70(30)$ \\
\hline IRAS22198 & 2.97 & $0.63(27)$ & & & & $0.56(25)$ \\
\hline IRAS23011 & 1.95 & $0.11(18)$ & $-0.81(28)$ & & & $-0.11(28)$ \\
\hline IRAS23385 & 4.73 & $2.01(17)$ & & & & 1.77(17) \\
\hline L483 & 1.11 & & $-1.91(89)$ & & & \\
\hline IRAS03282 & 0.08 & $-0.81(53)$ & $-2.37(98)$ & & & $-0.99(01)$ \\
\hline L1251B & 1.04 & $-0.80(17)$ & & & & $-0.75(35)$ \\
\hline Mean $^{c}$ & $4.7 \times 10^{5}$ & $359(103)$ & $79(21)$ & $64(24)$ & $77(18)$ & $272(94)$ \\
\hline Median $^{c}$ & $1.06 \times 10^{5}$ & 148 & 43 & 16 & 29 & 91 \\
\hline
\end{tabular}

Notes.

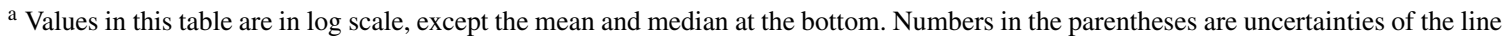
luminosity in the log scale, in the unit of 0.01 .

b Converted from Shirley et al. (2003).

${ }^{c}$ Statistics on massive dense clumps only, which does not include the sources below the horizontal line. Values and uncertainties (in the parentheses) are in the unit of $\mathrm{K} \mathrm{km} \mathrm{s}^{-1} \mathrm{pc}^{2}$.

et al. 2000). An excess was also found in a sample of lowmass starless cores (Gregersen \& Evans 2000). A recent study of somewhat more massive starless cores in Orion found no excess of blue-skewed profiles (Velusamy et al. 2008). While Velusamy et al. do not give masses of their cores, they are drawn from the sample of Li et al. (2007), which has a maximum mass of $46 M_{\odot}$. These studies all refer to what are now called cores, plausible precursors of single stars or small groups. The massive clumps in this study are much more massive and are likely to be the formation sites of clusters. In such sources, a blue-skewed profile would not reflect infall onto a forming star, but inflow toward the central parts of the clump.

The turbulence in these massive clumps makes detection of inflow motions difficult, but some sources have shown line profiles similar to those that are interpreted as evidence of inflow in lower mass cores. In particular, we found an excess of "blue" profiles over "red" profiles in initial observations of the HCN 3-2 line for this survey (Wu \& Evans 2003), and a similar result was found using $\mathrm{HCO}^{+}$by Fuller et al. (2005).

Since we have observed more sources with the HCN 3-2 line and an optically thin line (48) since the paper (Wu \& Evans 2003) on the statistics of blue profiles of 28 massive clumps, we can reassess this situation. The definitions of "blue" and "red" profiles have been discussed by Wu \& Evans (2003) and references therein. Here we note only that inflow may produce an asymmetric line profile in an optically thick line, skewing the peak toward the lower velocities relative to the peak of an optically thin line. This offset, measured in units of the width of an optically thin line, is defined by

$$
\delta v=\frac{v_{\text {thick }}-v_{\text {thin }}}{\delta v_{\text {thin }}} .
$$

An absolute value of $\delta v \geqslant 0.25$ is considered significant. In addition, the line profile may be double peaked with the blue peak stronger than the red peak. We refer to this signature as the blue peak. Since some profiles show the opposite asymmetries, one can characterize a sample by the excess, defined as

$$
E=\frac{N_{\text {blue }}-N_{\text {red }}}{N_{\text {total }}}
$$

We find that $\mathrm{H}^{13} \mathrm{CN} 3-2$ is already optically thick in some sources that show absorption features, while $\mathrm{C}^{34} \mathrm{~S} 5-4$ may be a better optically thin tracer for calculating $\delta v$. The mean ratio of the FWHM of $\mathrm{H}^{13} \mathrm{CN} 3-2$ to $\mathrm{C}^{34} \mathrm{~S} 5-4$ for our sample is $1.27 \pm$ 0.32 . So we use $\mathrm{C}^{34} \mathrm{~S} 5-4$ as the optically thin line to calculate $\delta v$ in this paper, and we use $\mathrm{H}^{13} \mathrm{CN} 3-2$ only when the $\mathrm{C}^{34} \mathrm{~S} 5-4$ line is not available. The $\delta v$ values are listed in Table 4. Using this measure, we identified 21 blue and 15 red profiles out of 48 sources; this leads to $E=0.13$ compared to $E=0.29$ in Wu \& Evans (2003). Using the blue peak method, we identified 16 blue and nine red profiles and $E=0.15$, compared to 0.29 in $\mathrm{Wu}$ \& Evans (2003). Thus, the inflow signature is not as common in the full sample as it was in the initial results. However, there are still more blue asymmetries than red, suggesting that mass inflow is occurring in at least some of the sample.

For comparison, Fuller et al. (2005) found values of $E$ of

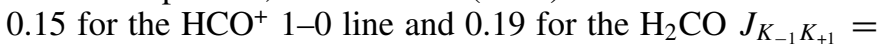
$2_{12} \rightarrow 1_{11}$ line in their study of 112 objects. They found no significant excess in the 3-2 or 4-3 lines of $\mathrm{HCO}^{+}$. There are only three sources in both the sample of Fuller et al. (2005) and our sample: S231, G59.78+0.06, and IRAS20106+3545 (called WFS2, 79, and 93 in Fuller's sample). In both samples, S231 was classified as a red profile and G59.78+0.06 as a blue profile (infall candidate). We do not have optically thin line data for IRAS20106, but its HCN 3-2 line show no asymmetry, which agrees with the observations by Fuller et al. (2005).

We also tabulate in Table 13 evidence for outflows based on the outflow catalog of $\mathrm{Wu}$ et al. (2005). Noted that this outflow catalog does not cover all the sources in our sample. It is a collective catalog that provides all the reported outflows by 

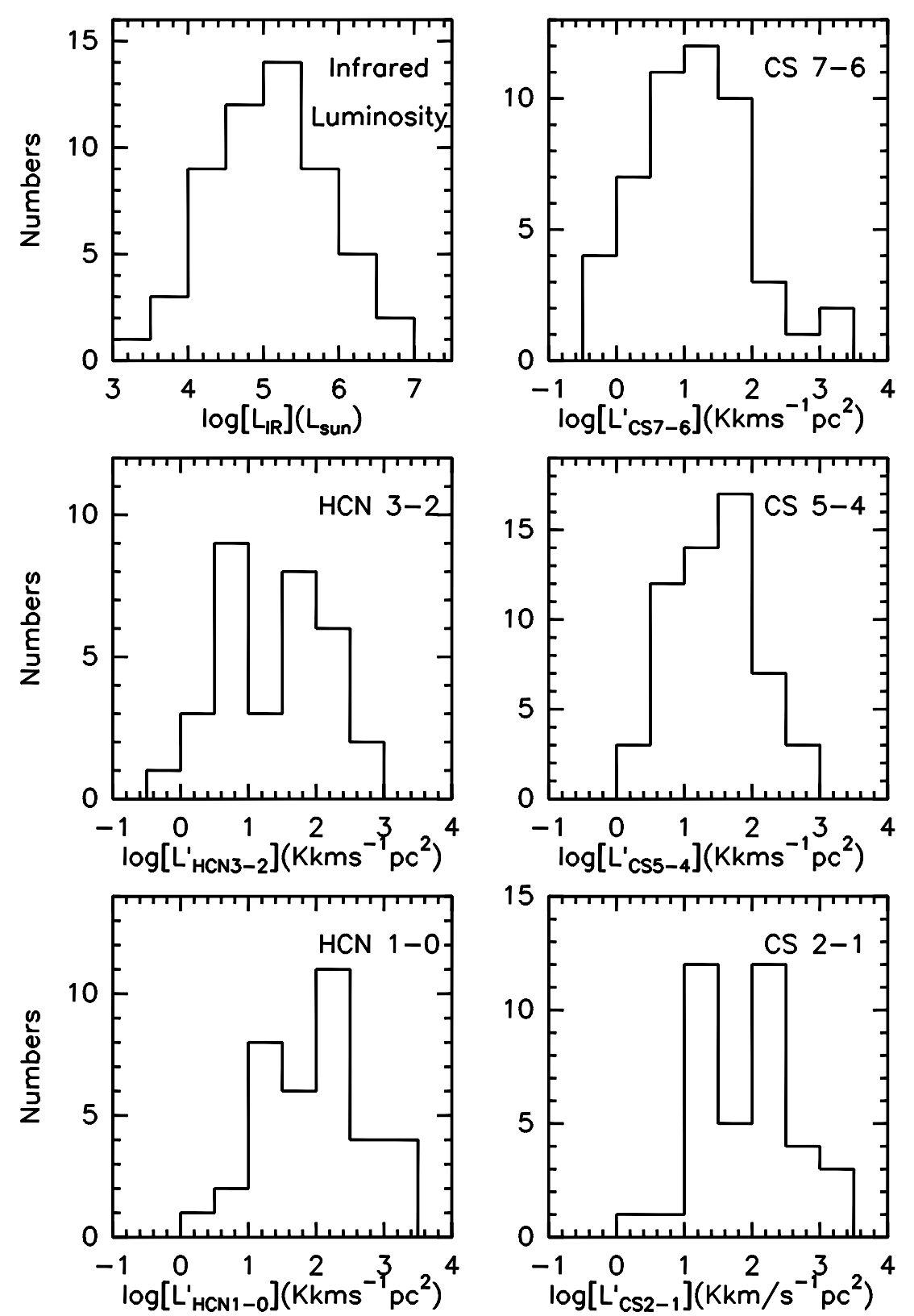

Figure 28. Histograms of the infrared and line luminosity of massive dense clumps in different transitions.

Table 8

Ratios of Sizes and Luminosities for Different Tracers ${ }^{\mathrm{a}}$

\begin{tabular}{lccccc}
\hline \hline Ratio & $\frac{R_{\mathrm{HCN} 1-0}}{R_{\mathrm{HCN} 3-2}}$ & $\frac{R_{\mathrm{CS} 2-1}}{R_{\mathrm{CS} 7-6}}$ & $\frac{R_{\mathrm{HCN} 1-0}}{R_{\mathrm{CS} 2-1}}$ & $\frac{R_{\mathrm{CS} 5-4}}{R_{\mathrm{CS} 7-6}}$ & $\frac{R_{\mathrm{HCN} 3-2}}{R_{\mathrm{CS} 5-4}}$ \\
\hline Mean $^{\mathrm{b}}$ & $2.97 \pm 0.19$ & $4.12 \pm 0.45$ & $1.11 \pm 0.03$ & $1.42 \pm 0.09$ & $1.11 \pm 0.04$ \\
Median $^{\mathrm{S}}$ & 2.71 & 3.36 & 1.07 & 1.31 & 1.05 \\
$\mathrm{Sdv}^{\mathrm{c}}$ & 1.06 & 2.40 & 0.23 & 0.64 & 0.21 \\
\hline & $L_{\mathrm{HCN} 1-0}^{\prime}$ & $\frac{L_{\mathrm{CS} 2-1}^{\prime}}{L_{\mathrm{CS} 7-6}}$ & $\frac{L_{\mathrm{CS} 2-1}}{L_{\mathrm{CS} 5-4}^{\prime}}$ & $\frac{L_{\mathrm{CS} 5-4}^{\prime}}{L_{\mathrm{CS} 7-6}^{\prime}}$ & $\frac{L_{\mathrm{HCN} 3-2}^{\prime}}{L_{\mathrm{CS} 5-4}^{\prime}}$ \\
\hline Mean $^{\mathrm{b}}$ & $4.3 \pm 0.4$ & $9.4 \pm 2.1$ & $4.0 \pm 0.4$ & $2.6 \pm 0.4$ & $1.6 \pm 0.1$ \\
Median $^{\prime}$ & 3.8 & 5.9 & 3.4 & 1.7 & 1.5 \\
$\mathrm{Sdv}^{\mathrm{c}}$ & 2.5 & 11.0 & 2.3 & 2.6 & 0.7 \\
\hline
\end{tabular}

Notes.

${ }^{\text {a }}$ Sizes of CS 5-4 maps come from Shirley et al. (2003).

${ }^{b}$ The uncertainty is the sigma of the mean.

${ }^{\mathrm{c}}$ The standard deviation of the ratios. 
Table 9

Properties of Different Transitions

\begin{tabular}{lccccccc}
\hline \hline Transition & $\begin{array}{c}n_{\text {crit }^{\mathrm{a}}} \\
(100 \mathrm{~K})\end{array}$ & $\begin{array}{c}E_{\mathrm{up}}{ }^{\mathrm{a}} \\
(\mathrm{K})\end{array}$ & $\begin{array}{c}n_{\mathrm{eff}^{\mathrm{a}}} \\
(100 \mathrm{~K})\end{array}$ & $\begin{array}{c}\text { Mean } L^{\prime} \\
\mathrm{K} \mathrm{km} \mathrm{s} \mathrm{pc}^{-2}\end{array}$ & $\begin{array}{c}\text { Mean } L_{\text {sub }}{ }^{\mathrm{c}} \\
\mathrm{K} \mathrm{km} \mathrm{s} \mathrm{pc}^{-2}\end{array}$ & $\begin{array}{c}\text { Mean R } \\
(\mathrm{pc})\end{array}$ & $\begin{array}{c}\mathrm{Mean} \mathrm{R}_{\text {sub }}{ }^{\mathrm{c}} \\
(\mathrm{pc})\end{array}$ \\
\hline CS 7-6 & $2.0 \times 10^{7}$ & 66 & $2.6 \times 10^{5}$ & 64 & 42 & 0.28 & 0.24 \\
CS 5-4 $^{\mathrm{b}}$ & $6.9 \times 10^{6}$ & 35 & $6.0 \times 10^{4}$ & 77 & 68 & 0.37 & 0.31 \\
HCN 3-2 & $6.8 \times 10^{7}$ & 26 & $3.6 \times 10^{4}$ & 79 & 101 & 0.32 & 0.35 \\
CS 2-1 & $3.9 \times 10^{5}$ & 7.1 & $4.1 \times 10^{3}$ & 272 & & 1.03 & \\
HCN 1-0 & $4.5 \times 10^{6}$ & 4.3 & $5.1 \times 10^{3}$ & 359 & & 1.13 & \\
\hline
\end{tabular}

Notes.

a From Evans (1999)

b From Shirley et al. (2003).

c Statistics on a subsample of the sources only, which have been mapped by all the three high- $J$ transitions CS 7-6, CS 5-4, and HCN 3-2.

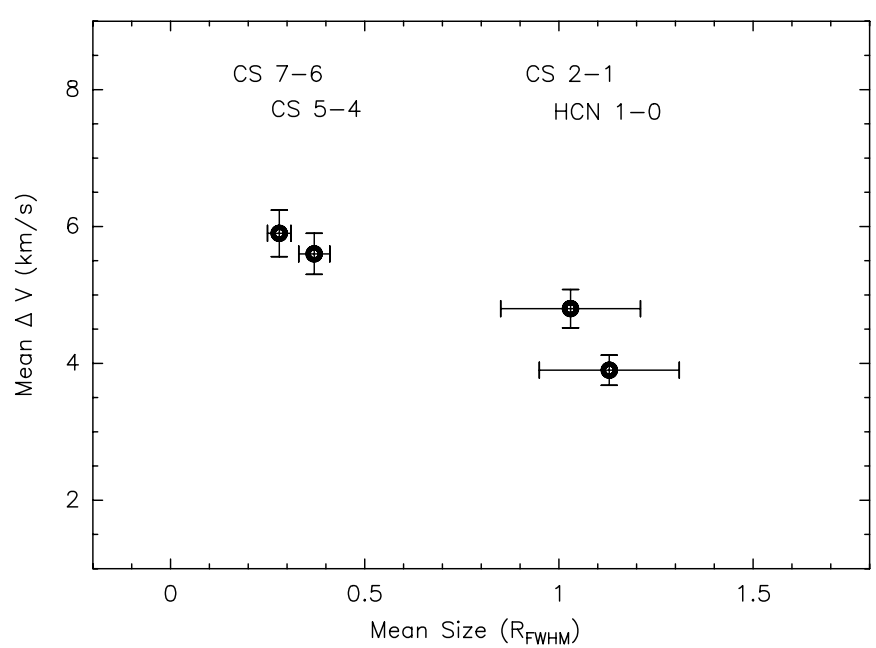

Figure 29. Inverse $\Delta V-R_{\mathrm{FWHM}}$ relation between different tracers for massive dense clumps.
2004. Therefore, the following statistics of outflows are based on the current available outflow reports, but not complete surveys. Usually outflow and infall signatures are not present in the same source, but there are obvious exceptions. Of the 46 sources whose $\delta v$ are available, outflow has been reported in 16 . Of these, eight have red asymmetry with $\delta v>0.20$, six have blue asymmetry with $\delta v<-0.20$, in which outflows and infall may occur at the same time. If we look the rate of the appearance of the outflow or infall signature as the signature of "dynamical activity," the rate of the sources that have infall or outflow to the total for each evolutionary stages are $55 \%$ for regions without $\mathrm{H}$ II regions, $66 \%$ for UCHII regions, $64 \%$ for $\mathrm{CHII}$ regions, and $33 \%$ for fully formed $\mathrm{H}$ II regions. These numbers suggest that the UCHII/CHII stages are more dynamically active than later stages.

\section{STAR FORMATION LAWS: CONNECTING TO EXTRAGALACTIC STAR FORMATION}

A linear $L_{\mathrm{IR}}-L_{\mathrm{HCN} 1-0}^{\prime}$ correlation has been reported (Gao \& Solomon 2004a) for nearby and distant starburst galaxies. A similar correlation has been found for Galactic clumps when study-
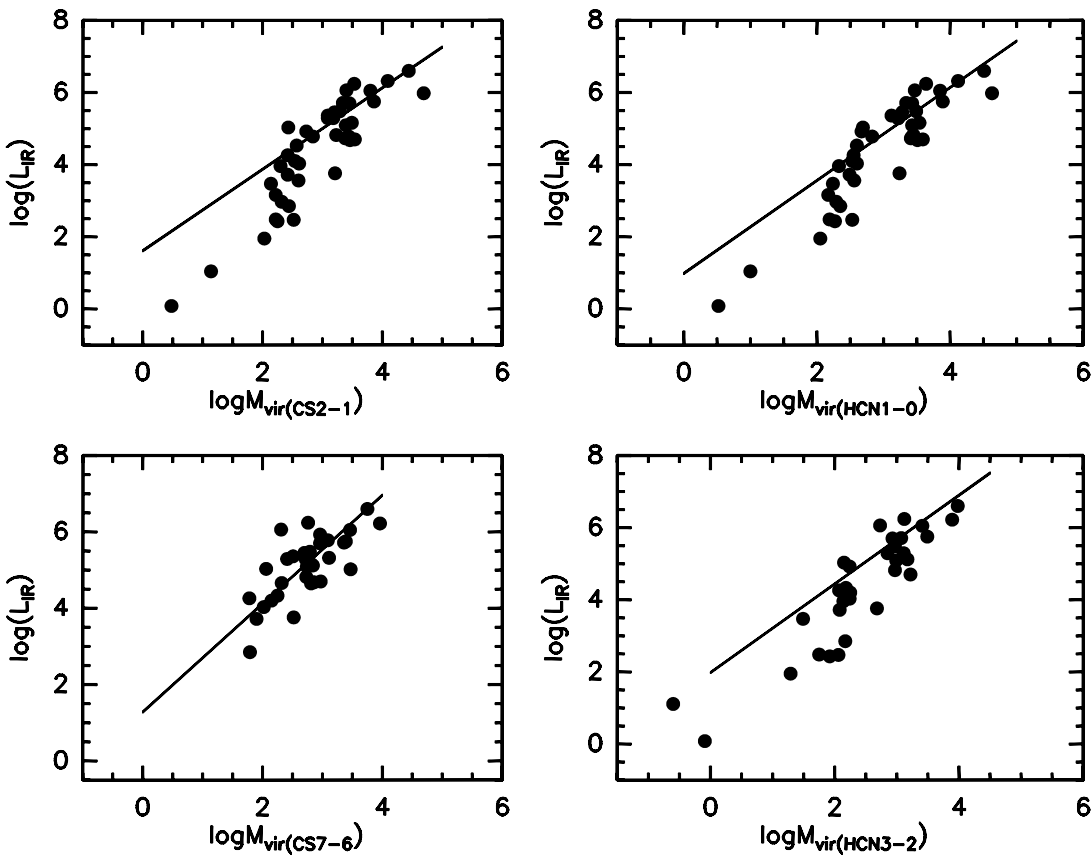

Figure 30. Correlations between the infrared luminosity and the virial mass measured by various dense gas tracers. The fitted lines show the linear least-squares fit for clumps with $L_{\mathrm{IR}}>10^{4.5} L_{\odot}$. 

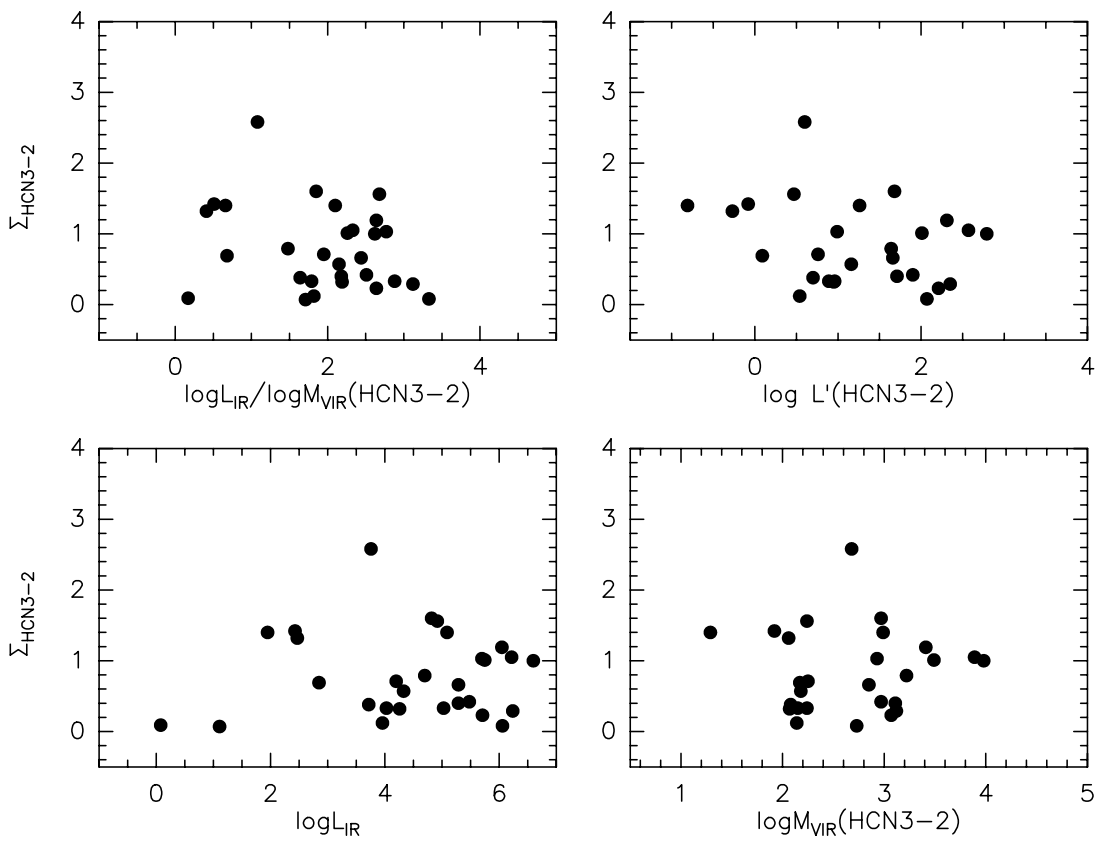

Figure 31. Plots of the surface density vs. the ratio of infrared luminosity to virial mass, infrared luminosity, line luminosity, and virial mass derived from HCN 3-2 maps.
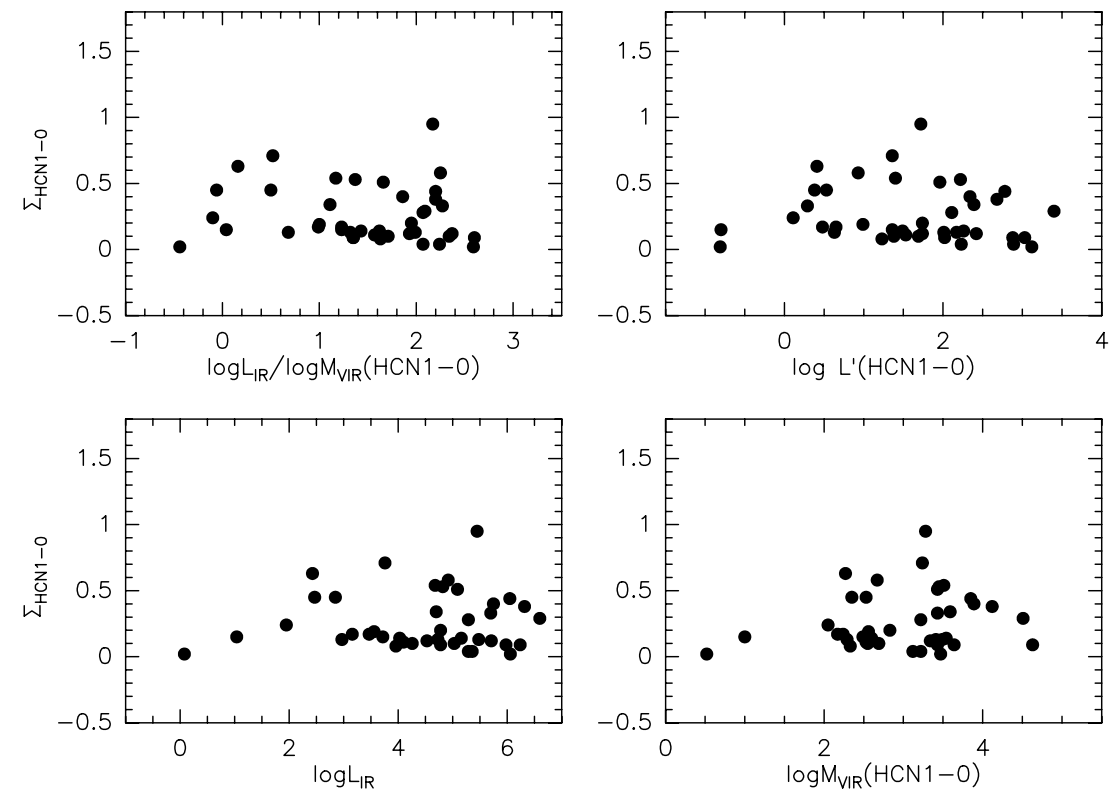

Figure 32. Plots of the surface density vs. the ratio of infrared luminosity to virial mass, infrared luminosity, line luminosity, and virial mass derived from HCN $1-0$ maps.

Table 10

Statistics of Line Width for Different Tracers

\begin{tabular}{lccccc}
\hline \hline Transitions & $\begin{array}{c}\Delta V(\mathrm{CS} \mathrm{7-6)} \\
\left(\mathrm{km} \mathrm{s}^{-1}\right)\end{array}$ & $\begin{array}{c}\Delta V(\mathrm{CS} \mathrm{5-4})^{\mathrm{a}} \\
\left(\mathrm{km} \mathrm{s}^{-1}\right)\end{array}$ & $\begin{array}{c}\Delta V(\mathrm{CS} \mathrm{2-1}) \\
\left(\mathrm{km} \mathrm{s}^{-1}\right)\end{array}$ & $\begin{array}{c}\Delta V(\mathrm{HCN} \mathrm{1-0)} \\
(F=2-1)\left(\mathrm{km} \mathrm{s}^{-1}\right)\end{array}$ & $\begin{array}{c}\Delta V\left(\mathrm{C}^{34} \mathrm{~S} 5-4\right) \\
\left(\mathrm{km} \mathrm{s}^{-1}\right)\end{array}$ \\
\hline Mean & $5.9 \pm 2.3$ & $5.6 \pm 2.2$ & $4.8 \pm 1.8$ & $3.9 \pm 1.0$ & $4.7 \pm 1.9$ \\
Median & 5.7 & 5.1 & 4.8 & 3.7 & 4.2 \\
\hline
\end{tabular}

Note.

${ }^{a}$ From Shirley et al. (2003).

ing CS 5-4 and dust emission maps (Shirley et al. 2003; Mueller et al. 2002). However, the study of the Kennicutt-Schmidt law with $\mathrm{CO}$ and $\mathrm{H}$ I shows a super-linear correlation (Kennicutt 1998; Kennicutt et al. 2007). Why are these two correlations different?
Two distinct explanations have been offered. Gao \& Solomon (2004a) and Wu et al. (2005) suggested that the line luminosity traces the mass of dense gas; the linear relation between $L_{\mathrm{IR}}$ and line luminosity then reflects the fact that the star formation rate is linearly proportional to the amount of gas above the threshold for 
Table 11

The Virial Mass, Surface Density, and Average Volume Density of Dense Clumps ${ }^{\mathrm{a}}$

\begin{tabular}{|c|c|c|c|c|c|c|c|c|c|c|c|c|}
\hline \multirow[t]{2}{*}{ Source } & \multicolumn{3}{|c|}{$\mathrm{HCN} 3 \rightarrow 2$} & \multicolumn{3}{|c|}{$\mathrm{HCN} 1 \rightarrow 0$} & \multicolumn{3}{|c|}{$\mathrm{CS} 7 \rightarrow 6$} & \multicolumn{3}{|c|}{$\mathrm{CS} 2 \rightarrow 1$} \\
\hline & $\begin{array}{c}\log \left(\mathrm{M}_{\mathrm{vir}}\right) \\
\left(M_{\odot}\right)\end{array}$ & $\begin{array}{c}\Sigma \\
\left(\mathrm{g} \mathrm{cm}^{-2}\right)\end{array}$ & $\log (\bar{n})$ & $\begin{array}{c}\log \left(\mathrm{M}_{\mathrm{vir}}\right) \\
\left(M_{\odot}\right)\end{array}$ & $\begin{array}{c}\Sigma \\
\left(\mathrm{g} \mathrm{cm}^{-2}\right)\end{array}$ & $\log (\bar{n})$ & $\begin{array}{c}\log \left(\mathrm{M}_{\mathrm{vir}}\right) \\
\left(M_{\odot}\right)\end{array}$ & $\begin{array}{c}\Sigma \\
\left(\mathrm{g} \mathrm{cm}^{-2}\right)\end{array}$ & $\log (\bar{n})$ & $\begin{array}{c}\log \left(\mathrm{M}_{\mathrm{vir}}\right) \\
\left(M_{\odot}\right)\end{array}$ & $\begin{array}{c}\Sigma \\
\left(\mathrm{g} \mathrm{cm}^{-2}\right)\end{array}$ & $\log (\bar{n})$ \\
\hline G121.30+0.66 & $2.17(12)$ & 0.69 & 5.54 & $2.35(10)$ & 0.45 & 5.19 & $1.79(16)$ & 1.65 & 6.31 & $2.44(11)$ & 0.37 & 5.01 \\
\hline G123.07-6.31 & $2.68(16)$ & 2.58 & 6.15 & $3.24(16)$ & 0.71 & 5.03 & $2.52(17)$ & 3.75 & 6.48 & $3.21(15)$ & 0.76 & 5.09 \\
\hline $\mathrm{W} 3(\mathrm{OH})$ & $2.99(05)$ & 1.40 & 5.60 & $3.43(03)$ & 0.51 & 4.72 & $2.74(05)$ & 2.54 & 6.11 & $3.39(05)$ & 0.56 & 4.80 \\
\hline $\mathrm{S} 231$ & $2.24(10)$ & 0.33 & 5.03 & $2.60(09)$ & 0.14 & 4.30 & $2.02(10)$ & 0.55 & 5.47 & $2.61(09)$ & 0.14 & 4.29 \\
\hline $\mathrm{S} 235$ & $2.08(09)$ & 0.38 & 5.21 & $2.49(09)$ & 0.15 & 4.38 & $1.90(10)$ & 0.58 & 5.57 & $2.42(08)$ & 0.18 & 4.53 \\
\hline $\mathrm{S} 255$ & $2.25(08)$ & 0.71 & 5.53 & & & & $2.15(10)$ & 0.91 & 5.74 & & & \\
\hline RCW142 & $2.97(06)$ & 1.60 & 5.70 & $3.45(06)$ & 0.53 & 4.74 & $2.73(03)$ & 2.73 & 6.16 & $3.23(05)$ & 0.87 & 5.17 \\
\hline W28A2(1) & & & & $3.28(04)$ & 0.95 & 5.20 & $2.70(01)$ & 3.66 & 6.37 & $3.20(04)$ & 1.15 & 5.36 \\
\hline M8E & $2.07(05)$ & 0.32 & 5.10 & $2.55(18)$ & 0.10 & 4.13 & $1.78(08)$ & 0.61 & 5.67 & $2.42(10)$ & 0.14 & 4.40 \\
\hline G9.62+0.19 & $3.49(04)$ & 1.01 & 5.14 & $3.89(06)$ & 0.40 & 4.34 & $3.39(10)$ & 1.26 & 5.33 & $3.86(12)$ & 0.43 & 4.40 \\
\hline G8.67-0.36 & $3.17(06)$ & 0.68 & 5.04 & & & & $2.84(06)$ & 1.45 & 5.70 & & & \\
\hline $\mathrm{W}(31)$ & $3.89(09)$ & 1.05 & 4.97 & & & & $3.96(18)$ & 0.89 & 4.82 & & & \\
\hline G10.60-0.40 & $3.41(02)$ & 1.19 & 5.28 & $3.85(03)$ & 0.44 & 4.42 & $3.46(03)$ & 1.06 & 5.18 & $3.80(04)$ & 0.49 & 4.51 \\
\hline G12.21-0.10 & & & & $4.63(24)$ & 0.09 & 2.98 & & & 7.42 & $4.69(16)$ & 0.08 & 2.85 \\
\hline G12.89+0.49 & & & & & & & $2.32(07)$ & 1.49 & 5.98 & & & \\
\hline W33cont & & & & & & & $3.36(03)$ & 0.45 & 4.68 & & & \\
\hline $\mathrm{G} 13.87+0.28$ & & & & $3.22(08)$ & 0.04 & 3.10 & $2.41(10)$ & 0.23 & 4.71 & $3.09(06)$ & 0.05 & 3.35 \\
\hline G14.33-0.64 & $2.18(11)$ & 0.57 & 5.42 & & & & $2.25(10)$ & 0.48 & 5.27 & & & \\
\hline $\mathrm{G} 23.95+0.16$ & & & & $3.12(23)$ & 0.04 & 3.18 & $2.51(13)$ & 0.15 & 4.40 & $3.09(22)$ & 0.04 & 3.24 \\
\hline W43S & $3.12(07)$ & 0.29 & 4.50 & $3.64(06)$ & 0.09 & 3.46 & $2.76(07)$ & 0.64 & 5.21 & $3.53(05)$ & 0.11 & 3.68 \\
\hline G31.41+0.31 & & & & & & & $3.11(05)$ & 1.39 & 5.54 & & & \\
\hline G31.44-0.26 & & & & & & & $3.09(11)$ & 0.26 & 4.45 & & & \\
\hline G32.05+0.06 & & & & & & & $3.47(21)$ & 0.70 & 4.91 & & & \\
\hline W44 & $2.97(09)$ & 0.42 & 4.82 & $3.49(13)$ & 0.13 & 3.78 & $2.79(08)$ & 0.63 & 5.18 & $3.29(08)$ & 0.20 & 4.18 \\
\hline G35.58-0.03 & & & & $3.51(21)$ & 0.54 & 4.72 & $2.86(14)$ & 2.41 & 6.02 & $3.46(11)$ & 0.61 & 4.82 \\
\hline G35.20-0.74 & & & & & & & $2.81(10)$ & 1.13 & 5.55 & & & \\
\hline W49S & & & & & & & $3.73(24)$ & 0.93 & 4.96 & & & \\
\hline G45.07+0.13 & $3.52(10)$ & 0.71 & 4.90 & $3.90(09)$ & 0.30 & 4.13 & $3.39(10)$ & 0.95 & 5.15 & $3.66(09)$ & 0.52 & 4.62 \\
\hline G48.61+0.02 & $2.73(13)$ & 0.08 & 3.88 & $3.47(07)$ & 0.02 & 2.41 & $2.31(14)$ & 0.22 & 4.72 & $3.40(14)$ & 0.02 & 2.54 \\
\hline W51W & & & & & & & $2.96(15)$ & 0.22 & 4.40 & & & \\
\hline W51M & $3.98(10)$ & 1.00 & 4.89 & $4.51(17)$ & 0.29 & 3.82 & $3.75(03)$ & 1.68 & 5.34 & $4.44(03)$ & 0.34 & 3.96 \\
\hline G59.78+0.06 & & & & $2.53(10)$ & 0.11 & 4.14 & & & & $2.54(11)$ & 0.10 & 4.13 \\
\hline S87 & & & & $2.60(06)$ & 0.12 & 4.18 & & & & $2.57(06)$ & 0.13 & 4.24 \\
\hline S88B & $2.15(10)$ & 0.33 & 5.07 & $2.69(09)$ & 0.10 & 4.00 & $2.06(09)$ & 0.41 & 5.27 & $2.43(06)$ & 0.17 & 4.52 \\
\hline K3-50 & & & & $4.12(07)$ & 0.38 & 4.18 & & & & $4.09(09)$ & 0.40 & 4.23 \\
\hline ON1 & & & & $3.54(06)$ & 0.14 & 3.80 & $2.81(12)$ & 0.73 & 5.26 & $3.49(07)$ & 0.15 & 3.91 \\
\hline ON2S & $3.07(09)$ & 0.23 & 4.37 & $3.34(08)$ & 0.12 & 3.84 & $2.97(07)$ & 0.29 & 4.58 & $3.34(07)$ & 0.12 & 3.84 \\
\hline $\mathrm{ON} 2 \mathrm{~N}$ & & & & & & & $2.86(11)$ & 0.40 & 4.85 & & & \\
\hline W75N & $2.85(07)$ & 0.66 & 5.18 & $3.22(03)$ & 0.28 & 4.44 & $2.72(04)$ & 0.88 & 5.43 & $3.18(06)$ & 0.31 & 4.53 \\
\hline DR21S & $2.93(03)$ & 1.03 & 5.43 & $3.43(03)$ & 0.33 & 4.43 & $2.96(06)$ & 0.97 & 5.38 & $3.45(05)$ & 0.31 & 4.39 \\
\hline W75OH & $3.22(10)$ & 0.79 & 5.12 & $3.59(05)$ & 0.34 & 4.37 & $2.97(06)$ & 1.40 & 5.61 & $3.54(04)$ & 0.37 & 4.46 \\
\hline CepA & $2.24(06)$ & 1.56 & 6.05 & $2.67(07)$ & 0.58 & 5.19 & & & & $2.73(07)$ & 0.50 & 5.07 \\
\hline S158 & $3.11(06)$ & 0.40 & 4.72 & & & & & & & & & \\
\hline S158A & $2.87(06)$ & 0.35 & 4.76 & & & & & & & & & \\
\hline IRAS20050+2720 & $2.06(29)$ & 1.32 & 6.03 & $2.53(26)$ & 0.45 & 5.08 & & & & $2.52(51)$ & 0.46 & 5.11 \\
\hline IRAS20106+3545 & & & & $2.17(24)$ & 0.17 & 4.64 & & & & $2.22(23)$ & 0.15 & 4.55 \\
\hline IRAS20126+4104 & & & & $2.83(15)$ & 0.20 & 4.40 & & & & $2.84(17)$ & 0.19 & 4.38 \\
\hline IRAS20188+3928 & $1.75(17)$ & 5.22 & 7.08 & $2.19(17)$ & 1.89 & 6.20 & & & & $2.22(17)$ & 1.76 & 6.13 \\
\hline IRAS20216+4107 & $1.49(34)$ & 0.96 & 6.10 & $2.24(20)$ & 0.17 & 4.62 & & & & $2.14(21)$ & 0.22 & 4.81 \\
\hline IRAS20220+3728 & & & & $3.43(20)$ & 0.09 & 3.61 & & & & $3.44(30)$ & 0.09 & 3.60 \\
\hline IRAS20333+4102 & $2.14(25)$ & 0.12 & 4.43 & $2.33(14)$ & 0.08 & 4.06 & & & & $2.30(15)$ & 0.08 & 4.11 \\
\hline IRAS21391+5802 & $1.92(20)$ & 1.42 & 6.14 & $2.27(16)$ & 0.63 & 5.44 & & & & $2.25(15)$ & 0.66 & 5.47 \\
\hline IRAS22172+5549 & & & & $2.56(21)$ & 0.19 & 4.51 & & & & $2.60(24)$ & 0.17 & 4.42 \\
\hline IRAS22198+6336 & & & & $2.29(21)$ & 0.13 & 4.42 & & & & $2.32(20)$ & 0.13 & 4.37 \\
\hline IRAS23011+6126 & $1.29(25)$ & 1.40 & 6.45 & $2.05(21)$ & 0.24 & 4.93 & & & & $2.03(24)$ & 0.25 & 4.97 \\
\hline IRAS23385+6053 & & & & $3.41(18)$ & 0.13 & 3.86 & & & & $3.38(18)$ & 0.14 & 3.91 \\
\hline IRAS03282+3035 & $-0.09(32)$ & 0.09 & 5.34 & $0.52(21)$ & 0.02 & 4.13 & & & & $0.48(24)$ & 0.02 & 4.19 \\
\hline L483 & $-0.06(27)$ & 0.07 & 5.44 & & & & & & & & & \\
\hline L1251B & & & & $1.00(09)$ & 0.15 & 5.13 & & & & $1.14(16)$ & 0.11 & 4.85 \\
\hline Mean $^{\text {b }}$ & 1555 & 0.78 & $2.5 \times 10^{5}$ & 5300 & 0.29 & $3.2 \times 10^{4}$ & 1300 & 1.09 & $1.7 \times 10^{6}$ & 5000 & 0.33 & $4.2 \times 10^{4}$ \\
\hline Sigma $^{b, c}$ & 445 & 0.55 & $0.7 \times 10^{5}$ & 1760 & 0.23 & $0.8 \times 10^{4}$ & 310 & 0.91 & $1.0 \times 10^{6}$ & 1870 & 0.27 & $1.0 \times 10^{4}$ \\
\hline Median $^{\mathrm{b}}$ & 890 & 0.68 & $1.3 \times 10^{5}$ & 2700 & 0.28 & $1.6 \times 10^{4}$ & 650 & 0.89 & $3.7 \times 10^{5}$ & 1950 & 0.31 & $2.6 \times 10^{4}$ \\
\hline
\end{tabular}

Notes.

a The virial mass, surface density, and volume density are calculated for mass within the FWHM contour of each transitions. Values for virial mass and volume density are in $\log$ scale, except the mean and median at bottom. Numbers in the parentheses are uncertainties in the log scale, in the unit of 0.01 .

b Statistics on massive dense clumps only, which does not include the sources below the horizontal line in the table.

${ }^{\mathrm{c}}$ The $\sigma$ of the virial mass is the $\sigma$ of the mean. 

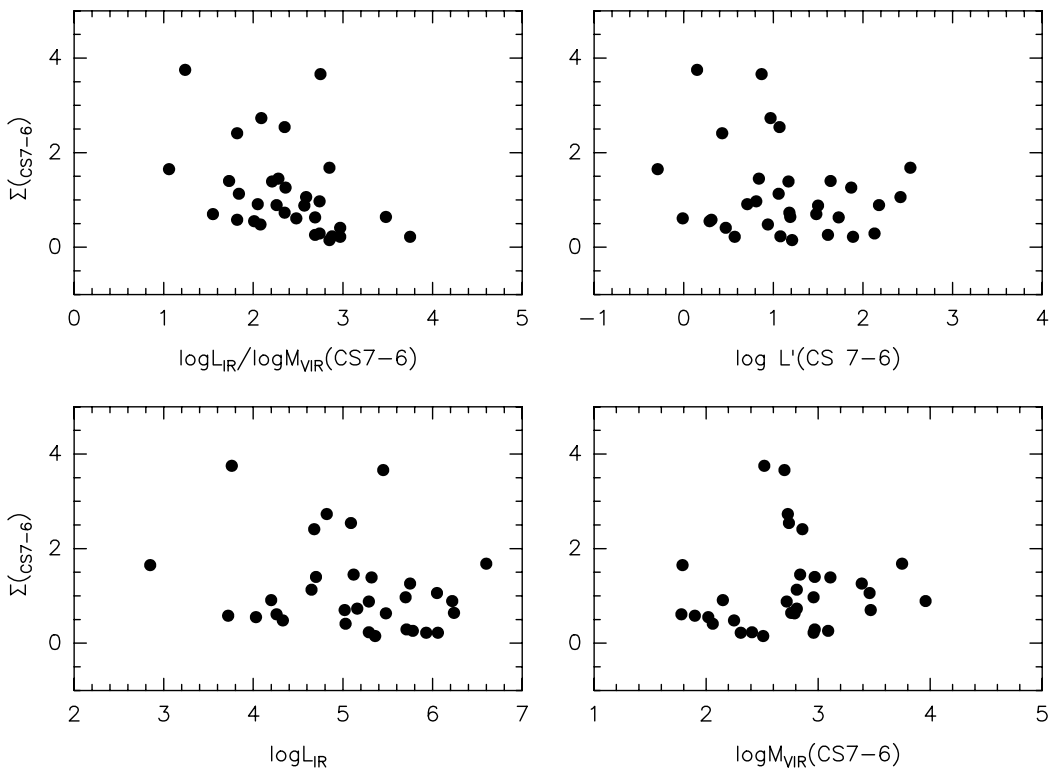

Figure 33. Plots of the surface density vs. the ratio of infrared luminosity to virial mass, infrared luminosity, line luminosity, and virial mass derived from CS 7-6 maps.
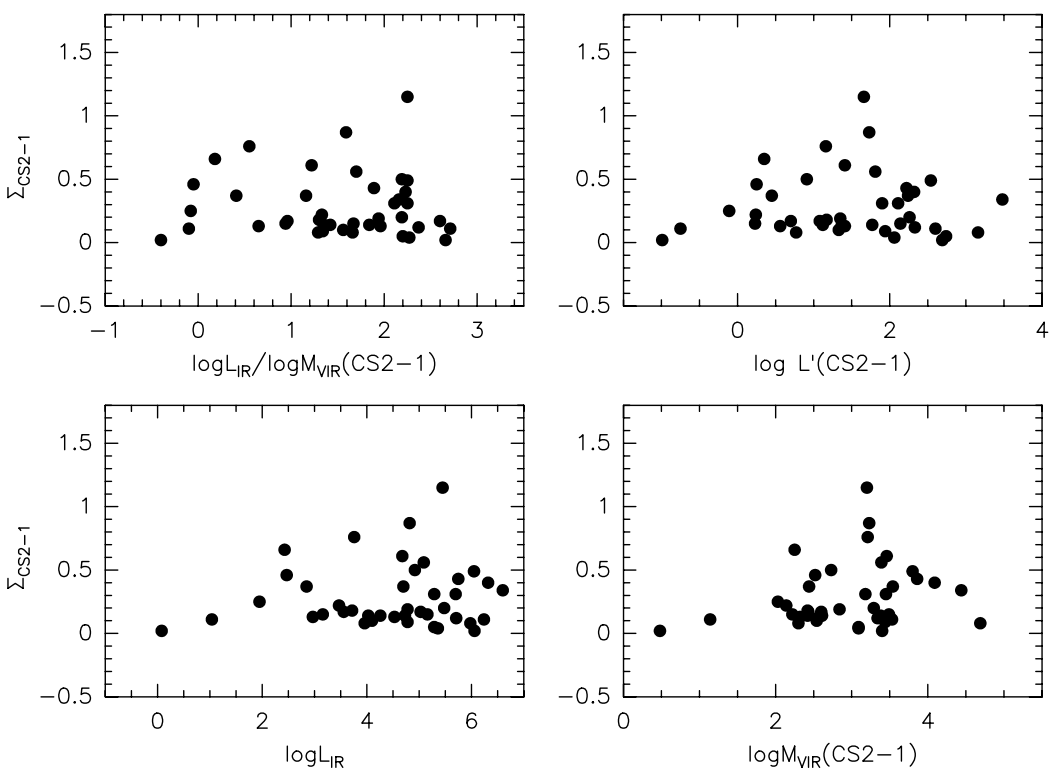

Figure 34. Plots of the surface density vs. the ratio of infrared luminosity to virial mass, infrared luminosity, line luminosity, and virial mass derived from CS $2-1$ maps.

star formation. The fact that massive dense clumps in our Galaxy lie on the same relationship seen for starburst galaxies indicates that starburst galaxies can be understood as large collections of dense clumps, such as we observe in our Galaxy. For a worked example of this idea, see Wu et al. (2009).

Krumholz \& Thompson (2007) and Narayanan et al. (2008) argue that the underlying star formation law is superlinear and the linear relations seen for dense gas tracers result from cancellation from a superlinear dependence of star formation rate on density. As explained by Narayanan et al. (2008), the following two relations,

$$
\begin{aligned}
& \text { SFR } \propto \rho^{N} \\
& L_{\text {mol }}^{\prime} \propto \rho^{\beta},
\end{aligned}
$$

can be combined to give

$$
\mathrm{SFR} \propto L_{\mathrm{mol}}^{\prime \alpha}
$$

with $\alpha=N / \beta$. In this picture, the linearity for dense gas tracers $(\alpha \sim 1)$ results from the coincidence that $N$ and $\beta$ have similar values. They predict that values of $\alpha$ will decline as higher excitation lines are used because $\beta$ is larger for subthermal lines. While Narayanan et al. (2008) are somewhat vague about the definition of $\rho$, it is generally discussed in terms of mean density along the line of sight, hence proportional to surface density.

The current surveys allow us to examine the correlations for dense clumps in our Galaxy for five different tracers of dense gas. For tracers with sufficient data on other galaxies, we can compare the Galactic data on individual clumps to that for other galaxies.

\subsection{What do $L_{\mathrm{mol}}^{\prime}$ Measurements Trace?}

A first question is whether $L_{\mathrm{HCN} 1-0}^{\prime}$ traces the mass of dense gas, as suggested by Gao \& Solomon (2004a). We plot the $L_{\mathrm{HCN} 1-0}^{\prime}-M_{\mathrm{VIR}}\left(R_{\mathrm{HCN} 1-0}\right)$ correlation in Figure 43 . For comparison, we also plot the $L_{\text {molecule versus }} M_{\mathrm{VIR}}\left(R_{\text {molecule }}\right)$ for 

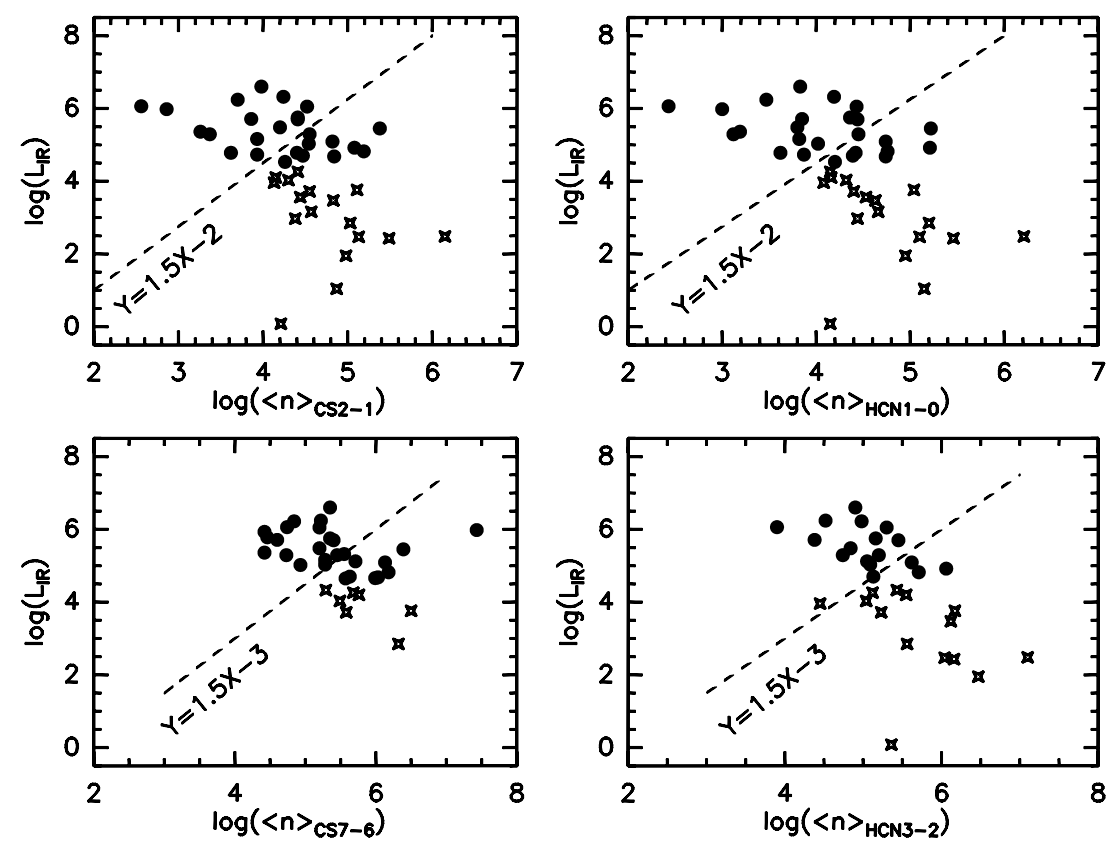

Figure 35. Correlation between infrared luminosity and the mean volume density $\langle n\rangle$ ( $\bar{n}$ in the text) within the FWHM contour of CS $2-1$, CS 7-6, HCN $1-0$, and HCN 3-2 maps. The dashed line has the slope predicted by Krumholz \& McKee (2008) and Narayanan et al. (2008) and an arbitrary offset. The clumps with $L_{\text {IR }}$ above and below the threshold luminosity $10^{4.5} L_{\odot}$ are plotted with solid circles and hollow stars, respectively.
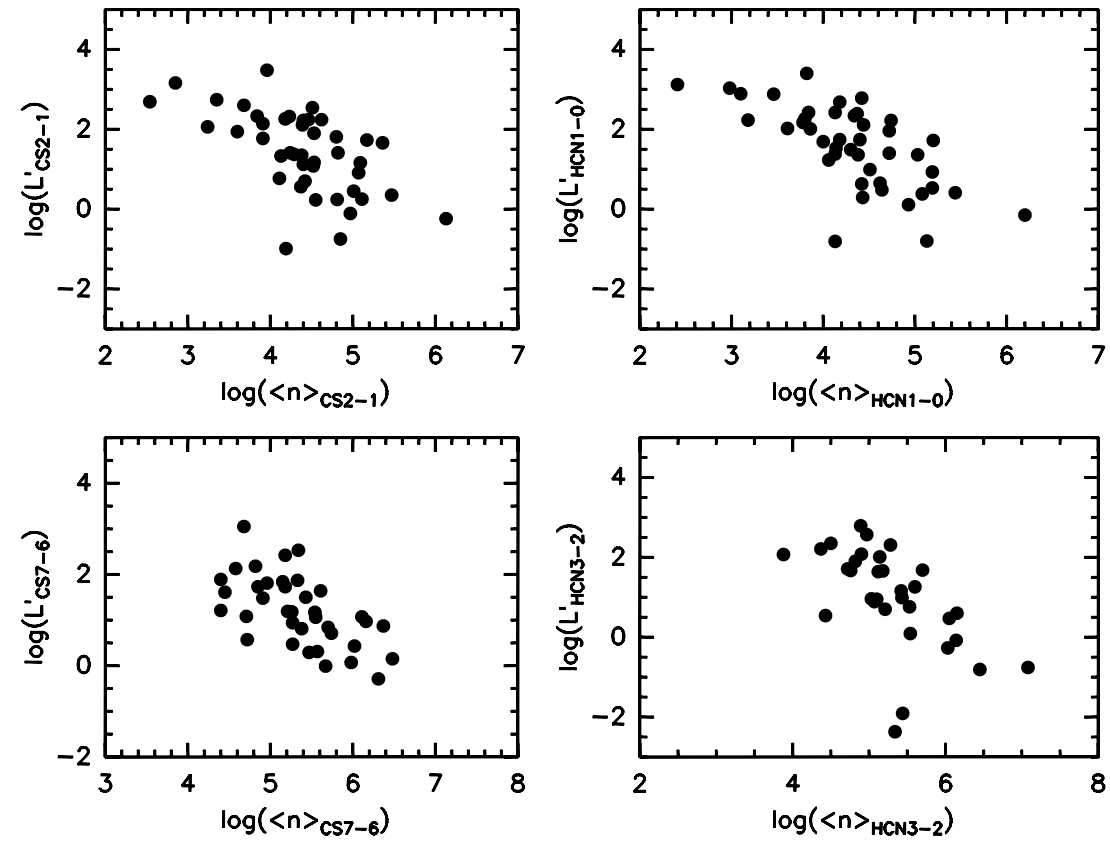

Figure 36. Correlation between line luminosity and the mean volume density $\langle n\rangle(\bar{n}$ in the text) within the FWHM contour of CS $2-1$, CS $7-6$, HCN $1-0$, and HCN 3-2 maps.

the other four dense gas tracers in Figure 43. For all the panels, we show fits based on least squares minimization (dashed line) and robust estimation (solid line). Here are the results from the fits:

HCN 1-0:

Robust Fit : $\log \left(L_{\mathrm{HCN} 1-0}^{\prime}\right)=1.04 \times \log \left(M_{\mathrm{Vir}}\left(R_{\mathrm{HCN} 1-0}\right)\right)-1.35$

Least squares : $\log \left(L_{\mathrm{HCN} 1-0}^{\prime}\right)=1.17( \pm 0.05)$

$\times \log \left(M_{\mathrm{Vir}}\left(R_{\mathrm{HCN} 1-0}\right)\right)-1.78( \pm 0.16) ; r=0.91$
HCN 3-2:

Robust fit : $\log \left(L_{\mathrm{HCN} 3-2}^{\prime}\right)=1.17 \times \log \left(M_{\mathrm{Vir}}\left(R_{\mathrm{HCN} 3-2}\right)\right)-1.89$

Least squares : $\log \left(L_{\mathrm{HCN} 3-2}^{\prime}\right)=1.33( \pm 0.06)$

$$
\times \log \left(M_{\mathrm{Vir}}\left(R_{\mathrm{HCN} 3-2}\right)\right)-2.36( \pm 0.18) ; r=0.93
$$

CS 2-1:

Robust fit : $\log \left(L_{\mathrm{CS} 2-1}^{\prime}\right)=1.06 \times \log \left(M_{\mathrm{Vir}}\left(R_{\mathrm{CS} 2-1}\right)\right)-1.50$

Least squares : $\log \left(L_{\mathrm{CS} 2-1}^{\prime}\right)=1.26( \pm 0.05)$

$$
\times \log \left(M_{\mathrm{Vir}}\left(R_{\mathrm{CS} 2-1}\right)\right)-2.17( \pm 0.16) ; r=0.91
$$




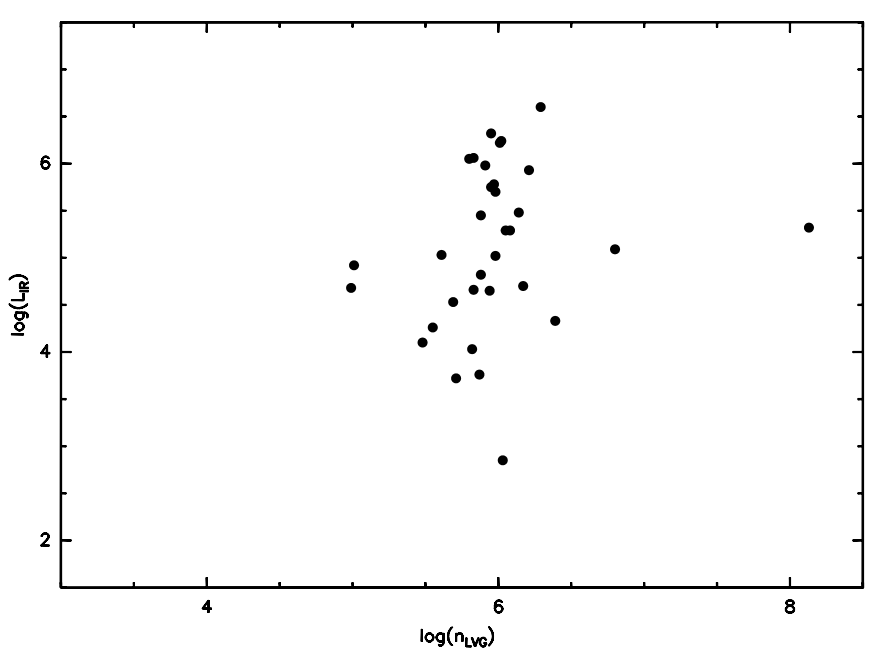

Figure 37. $L_{\mathrm{IR}}$ vs. volume density derived from the LVG model (Plume et al. 1997).

\section{CS 5-4:}

Robust fit : $\log \left(L_{\mathrm{CS} 5-4}^{\prime}\right)=0.95 \times \log \left(M_{\mathrm{Vir}}\left(R_{\mathrm{CS} 5-4}\right)\right)-1.26$

$$
\begin{aligned}
& \text { Least squares }: \log \left(L_{\mathrm{CS} 5-4}^{\prime}\right)=1.26( \pm 0.06) \\
& \quad \times \log \left(M_{\text {Vir }}\left(R_{\mathrm{CS} 5-4}\right)\right)-2.23( \pm 0.17) ; r=0.85
\end{aligned}
$$

CS 7-6:

$$
\text { Robust fit : } \log \left(L_{\mathrm{CS} 7-6}^{\prime}\right)=1.03 \times \log \left(M_{\mathrm{Vir}}\left(R_{\mathrm{CS} 2-1}\right)\right)-1.66
$$

$$
\begin{aligned}
& \text { Least squares }: \log \left(L_{\mathrm{CS} 7-6}^{\prime}\right)=1.64( \pm 0.05) \\
& \times \log \left(M_{\mathrm{Vir}}\left(R_{\mathrm{CS7}-6}\right)\right)-3.37( \pm 0.15) ; r=0.83
\end{aligned}
$$

The robust estimation fit consistently gives values near unity, indicating linear relations between $L_{\mathrm{mol}}^{\prime}$ and $M_{\mathrm{Vir}}$. The least squares method yields slight superlinear relations, with values around 1.25, except for CS 7-6. More CS 7-6 maps on the lower luminosity may help to clarify which fit is better. More importantly, these correlations are by far the strongest we have plotted so far, confirming that $L_{\mathrm{mol}}^{\prime}$ is an excellent tracer of $M_{\mathrm{Vir}}$ for the dense gas.

In Table 14, we give the statistics on the ratios of logarithms of the virial mass to line luminosity for each tracer. The scatter is about a factor of 3 . All these lines provide good tracers for the mass of dense gas, as required for the Gao \& Solomon interpretation. That statement is true even though these tracers are optically thick. The explanation is probably similar to explanations given for the fact that $\mathrm{CO}$ traces the mass of lower density material. Because $\mathrm{CO}$ is thermalized at densities above about $1000 \mathrm{~cm}^{-3}$ it is not sensitive to the dense gas that we are studying with these tracers. Questions have been raised about the ability of $\mathrm{HCN}$ and CS to trace the mass because they require high densities to excite (Krumholz \& Thompson 2007; Narayanan et al. 2008). In our view, that is exactly their virtue: they trace only the dense gas, which $\mathrm{CO}$ can fail to trace. The density of the material they are tracing depends on their sensitivity to density and temperature, as discussed above. What is clear is that they trace the regions where clusters of stars, including massive stars, are forming and are thus most relevant to estimating the mass of gas that is immediately relevant for star formation.

The good correlation between $L_{\mathrm{mol}}^{\prime}$ and $M_{\mathrm{Vir}}$ stands in sharp contrast to the lack of correlation between surface density (a good tracer for the mean density of the clump) and $L_{\text {mol }}^{\prime}$ (Figures 31-34). The spread in $L_{\text {mol }}^{\prime}$ for a given surface density is about three orders of magnitude. We see no evidence in the data for the relation between $L_{\mathrm{mol}}^{\prime}$ and mean density posited by Narayanan et al. (2008).

\subsection{Relations between $L_{\mathrm{IR}}$ and $L_{\mathrm{mol}}^{\prime}$}

The next question is whether the relations between $L_{\mathrm{IR}}$ and $L_{\text {mol }}^{\prime}$ seen in other galaxies (Gao \& Solomon 2004a) extend down to the scale of individual clumps in our Galaxy. The initial results of our survey of $\mathrm{HCN} 1-0$ indicated that they did, except
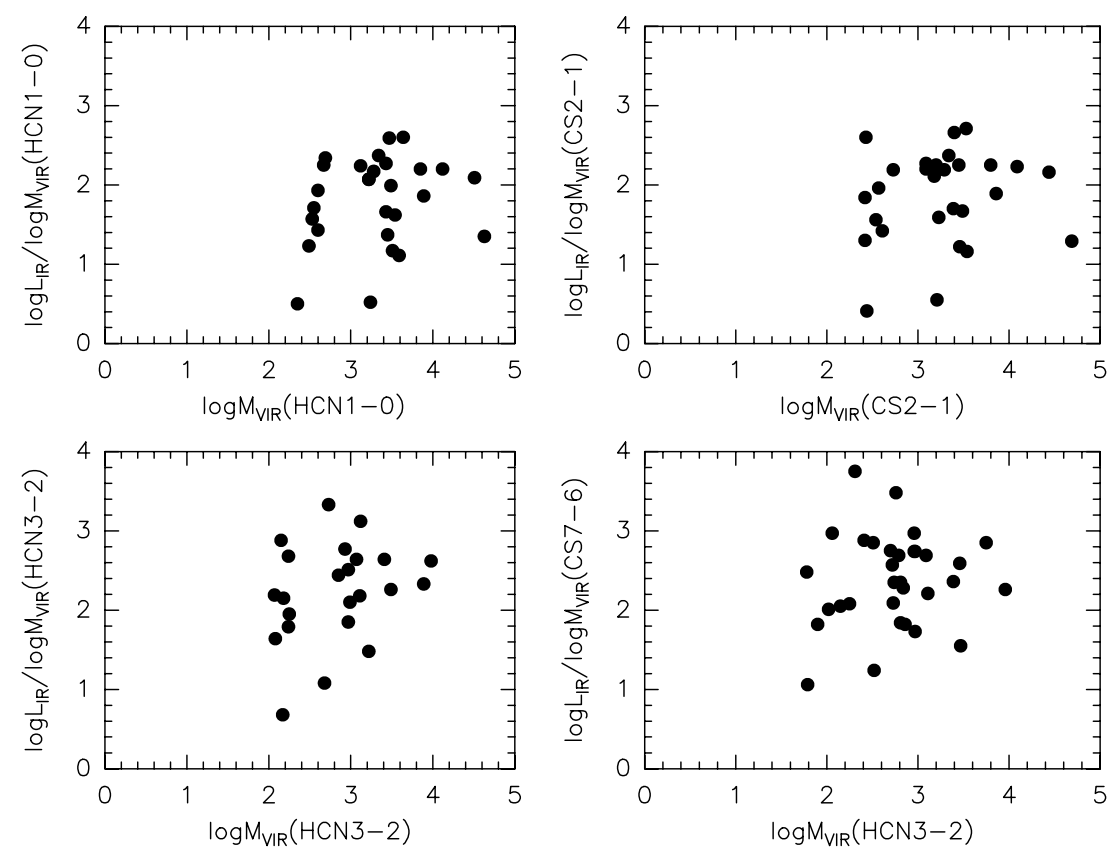

Figure 38. Star formation efficiency indicated by the infrared luminosity per unit of dense gas mass vs. virial mass. 

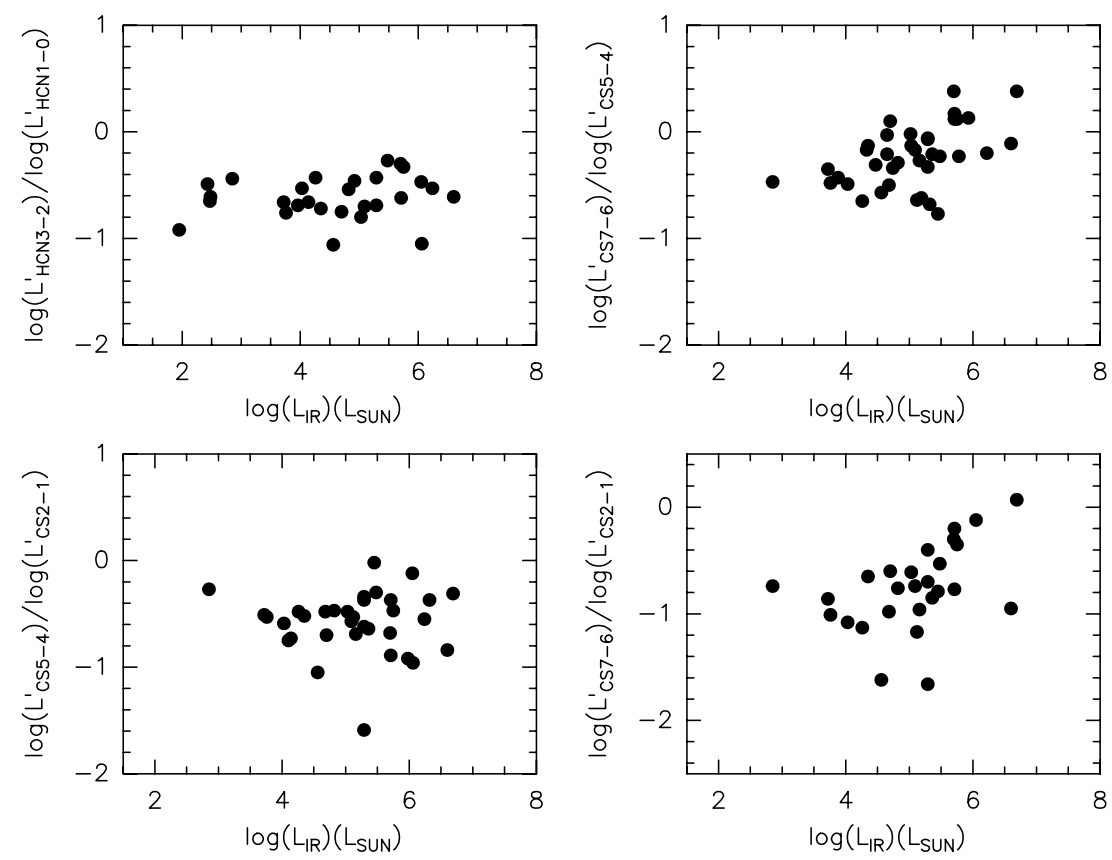

Figure 39. Luminosity ratios between different $J$ transitions vs. infrared luminosity.
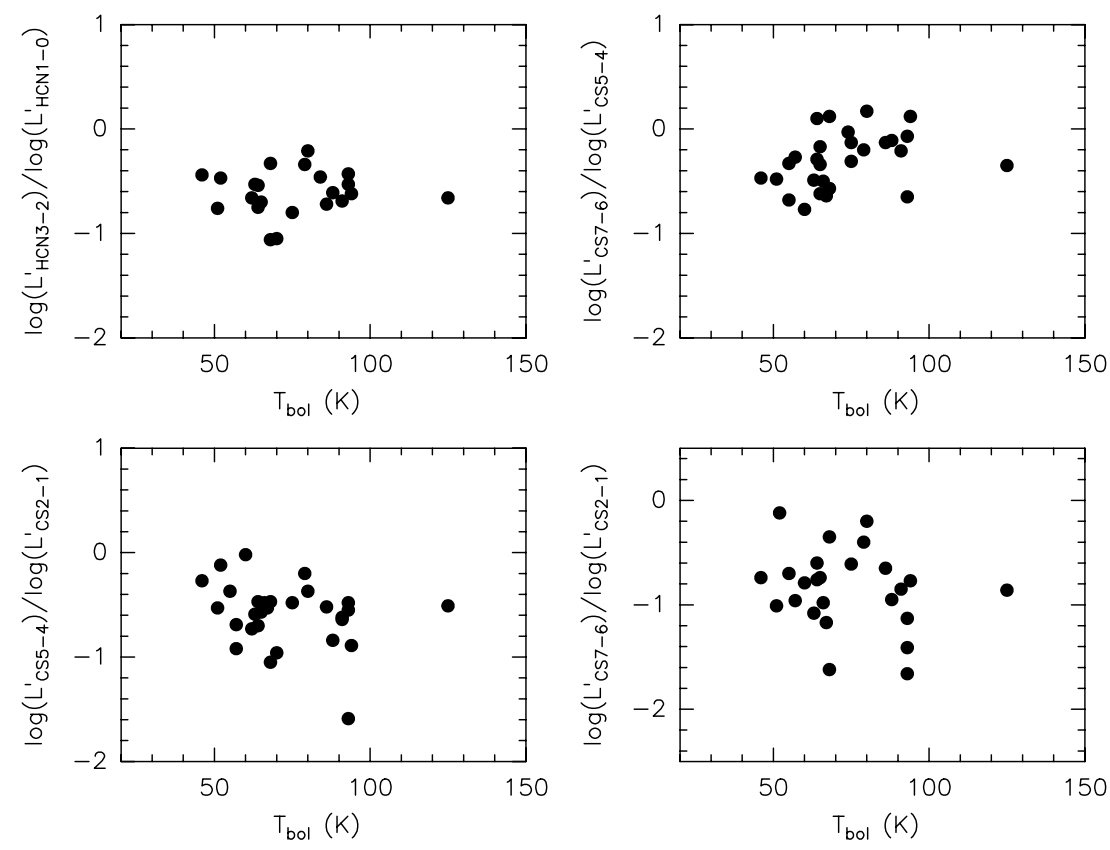

Figure 40. Luminosity ratios between different $J$ transitions vs. bolometric temperature.

that there is a threshold, below which there is not enough dense gas to sample the IMF. Because $L_{\mathrm{IR}}$ is dominated by massive stars, it can trace the star formation rate in the same way as galaxies, only with a reasonable sampling of the IMF. Even then, it will be subject to large statistical fluctuations from clump to clump, and it may underestimate the star formation rate because it takes some time for massive stars to form (Krumholz \& Thompson 2007). Therefore, we must expect substantial scatter in this relationship.

With these caveats in mind, we can ask two questions. Does $L_{\mathrm{IR}}$ correlate with $L_{\mathrm{mol}}^{\prime}$ ? Does the relation steepen for tracers with higher critical density, as predicted by Narayanan et al. (2008)? We now consider these questions for the four tracers in this survey, as well as CS 5-4 mapping survey from Shirley et al. (2003). In Figure 44, we plot $L_{\mathrm{IR}}$ versus $L_{\mathrm{mol}}^{\prime}$ for $\mathrm{HCN}$ 1-0, HCN 3-2, CS 2-1, CS 5-4 and CS 7-6. Figure 45 shows the correlation between the distance independent ratio $L_{\mathrm{IR}} / L_{\mathrm{mol}}^{\prime}$, an indicator of star formation efficiency, and $L_{\mathrm{IR}}$ for different tracers. Extragalactic data are lacking for the CS transitions, so we show the extragalactic data only for the HCN transitions. It is important to note that $L_{\mathrm{IR}}$ is the same for each tracer, as it is a property of the entire clump and is measured from emission in large beams (e.g., IRAS) or by integrating over maps at submillimeter wavelengths. This point has been missed by some analysts.

First we consider the plots using $L_{\mathrm{HCN}}^{\prime}(1-0)$ as the dense gas tracer. The correlation between $L_{\mathrm{IR}}$ and $L_{\mathrm{HCN}}^{\prime}(1-0)$ found in the galaxies extends to the scale of dense clumps in our Galaxy, as 

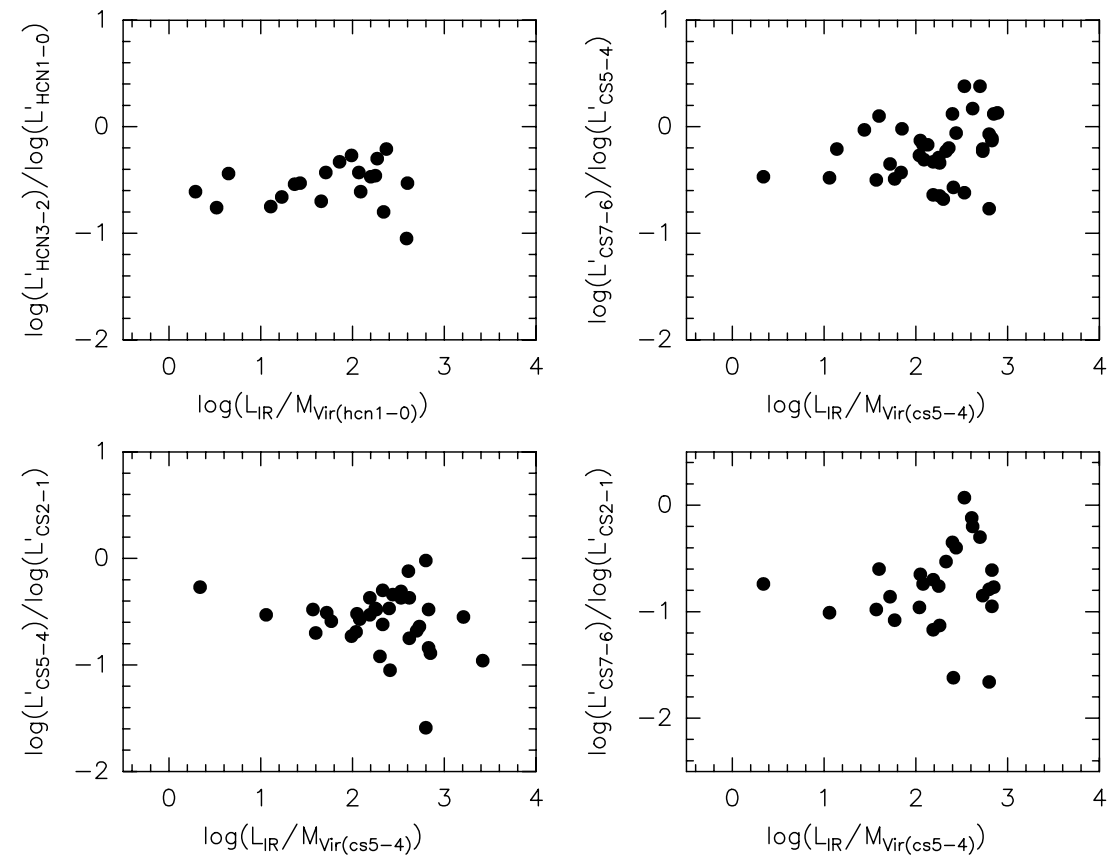

Figure 41. Luminosity ratios between different $J$ transitions vs. star formation efficiency.

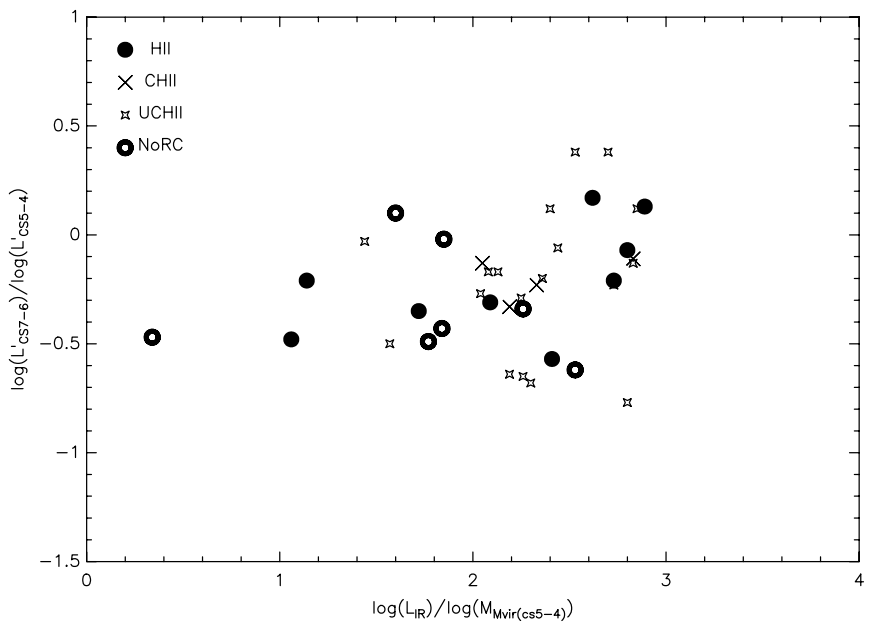

Figure 42. Distribution of clumps at different evolutionary states in the line luminosity ratio vs. star formation efficiency plot.

originally found by Wu et al. (2005). There is a threshold $L_{\text {mol }}^{\prime}$, or equivalently, $L_{\mathrm{IR}}$, which is most easily seen in Figure 45. For $L_{\mathrm{IR}}>10^{4.5} L_{\odot}$, all dense gas tracers show a nearly linear correlation with $L_{\mathrm{IR}}$. Above the threshold, a linear least-squares fit with uncertainties in both axes gives

$$
\begin{aligned}
\log \left(L_{\mathrm{IR}}\right)= & 1.07( \pm 0.06) \times \log \left(L_{\mathrm{HCN} 1-0}^{\prime}\right) \\
& +2.98( \pm 0.14) ; r=0.85
\end{aligned}
$$

and a robust fit gives

$$
\left(L_{\mathrm{IR}}\right)=1.03 \times \log \left(L_{\mathrm{HCN} 1-0}^{\prime}\right)+3.12 .
$$

The fit to this relation in other galaxies is shown in Figure 44; it is remarkably similar to that for massive, dense clumps in our Galaxy.

Data for other lines in galaxies are scarce. The other panels in Figures 44 and 45 show the relations for the clumps in this study, along with the fit for the HCN $1-0$ line in other galaxies.
Table 12

Statistics of Line Ratio $\log \left(\frac{\left(L_{\mathrm{CS} 7-6}^{\prime}\right)}{\left(L_{\mathrm{CS} 5-4}^{\prime}\right)}\right)$ by Evolutionary Stages ${ }^{\mathrm{a}}$

\begin{tabular}{lcccc}
\hline \hline \multicolumn{1}{c}{ Stage } & No H II region & UCHII & Comp. H II & H II \\
\hline Mean $^{\mathrm{b}}$ & $-0.45 \pm 0.08$ & $-0.21 \pm 0.08$ & $-0.40 \pm 0.15$ & $-0.21 \pm 0.08$ \\
Median $^{\text {Std. Dev. }}{ }^{\mathrm{c}}$ & -0.47 & -0.20 & -0.28 & -0.21 \\
St & 0.34 & 0.37 & 0.41 & 0.25 \\
\hline
\end{tabular}

Notes.

${ }^{a}$ Luminosities of CS 5-4 come from Shirley et al. (2003).

$\mathrm{b}$ The uncertainty is the sigma of the mean.

$\mathrm{c}$ The standard deviation of the ratios.

All show correlations with $L_{\mathrm{IR}}$ above the threshold. We give here (in order of increasing $n_{\text {crit }}$ ) the linear least-squares fit with uncertainties in both axes, and the robust fit for other dense gas tracers for cores with $L_{\mathrm{IR}}>10^{4.5} L_{\odot}$.

CS 2-1:

$$
\text { Least squares : } \begin{aligned}
\log \left(L_{\mathrm{IR}}\right)= & 1.03( \pm 0.05) \times \log \left(L_{\mathrm{CS} 2-1}^{\prime}\right) \\
& +3.25( \pm 0.11) ; r=0.80
\end{aligned}
$$

Robust fit : $\log \left(L_{\mathrm{IR}}\right)=0.87 \times \log \left(L_{\mathrm{CS} 2-1}^{\prime}\right)+3.56$

CS 5-4:

Least squares fit : $\log \left(L_{\mathrm{IR}}\right)=1.05( \pm 0.05) \times \log \left(L_{\mathrm{CS} 5-4}^{\prime}\right)$ $+3.77( \pm 0.08) ; r=0.86$

Robust fit : $\log \left(L_{\mathrm{IR}}\right)=0.86 \times \log \left(L_{\mathrm{CS} 5-4}^{\prime}\right)+3.90$

CS 7-6:

Least squares fit : $\log \left(L_{\mathrm{IR}}\right)=0.81( \pm 0.04) \times \log \left(L_{\mathrm{CS} 7-6}^{\prime}\right)$ $+4.31( \pm 0.06) ; r=0.81$

Robust fit : $\log \left(L_{\mathrm{IR}}\right)=0.64 \times \log \left(L_{\mathrm{CS} 7-6}^{\prime}\right)+4.58$ 
Table 13

Evolutionary Analysis of Massive Clumps

\begin{tabular}{|c|c|c|c|c|c|c|}
\hline Source & $\mathrm{H}_{\mathrm{II}} ?^{\mathrm{a}}$ & 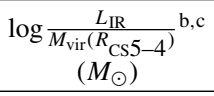 & $\log \frac{L^{\prime}(\mathrm{HCN} 3-2) \mathrm{b}}{L^{\prime}(\mathrm{HCN} 1-0)}$ & $\log \frac{L^{\prime}(\mathrm{CS} 7-6) \mathrm{b}, \mathrm{c}}{L^{\prime}(\mathrm{CS} 5-4)}$ & Outflow? ${ }^{\mathrm{d}}$ & Infall? ${ }^{e}$ \\
\hline G121.30+0.66 & & 0.34 & -0.44 & -0.47 & $\mathrm{Y}$ & $\mathrm{Y}$ \\
\hline $\mathrm{S} 231$ & & 1.77 & -0.53 & -0.49 & $\mathrm{Y}$ & \\
\hline S241 & & 1.99 & -0.66 & & $\mathrm{Y}$ & \\
\hline $12.89+0.49$ & & 2.13 & & -0.96 & & \\
\hline W33A & & 2.53 & & -0.62 & & \\
\hline $24.49-0.04$ & & 2.26 & & -0.34 & & \\
\hline $28.86+0.07$ & & 2.68 & & & & \\
\hline W43 Main3 & & & & -0.78 & & \\
\hline $32.05+0.20$ & & 1.85 & & -0.02 & & $\mathrm{Y}$ \\
\hline W75(OH) & & 1.60 & -0.66 & 0.10 & & $\mathrm{Y}$ \\
\hline BFS 11-B & & 1.84 & & -0.43 & $\mathrm{Y}$ & \\
\hline $\operatorname{Mean}^{\mathrm{f}}$ & & $2.02(0.12)$ & $-0.62(0.04)$ & $-0.45(0.11)$ & & \\
\hline Median & & 1.99 & -0.66 & -0.47 & & \\
\hline W3(OH) & UCHII & 2.08 & -0.70 & -0.17 & & $\mathrm{Y}$ \\
\hline S255 & UCHII & & & & $\mathrm{Y}$ & \\
\hline RCW142 & UCHII & 2.25 & -0.54 & -0.29 & & $\mathrm{Y}$ \\
\hline W28A2(1) & UCHII & 2.80 & & -0.77 & $\mathrm{Y}$ & \\
\hline M8E & UCHII & 2.26 & -0.43 & -0.65 & $\mathrm{Y}$ & \\
\hline $9.62+0.19$ & UCHII & 2.40 & -0.24 & 0.12 & $\mathrm{Y}$ & \\
\hline $8.67-0.36$ & UCHII & 2.19 & & -0.64 & & $\mathrm{Y}$ \\
\hline W31(1) & UCHII & 2.36 & & -0.20 & & $\mathrm{Y}$ \\
\hline $12.42+0.50$ & UCHII & & & & & $\mathrm{Y}$ \\
\hline W33cont & UCHII & & & 0.51 & & \\
\hline $14.33-0.64$ & UCHII & 2.13 & & -0.17 & & \\
\hline $20.08-0.13$ & UCHII & 1.44 & & -0.03 & & \\
\hline W42 & UCHII & & & -0.51 & & \\
\hline $31.41+0.31$ & UCHII & 2.30 & & -0.68 & & $\mathrm{Y}$ \\
\hline $31.44-0.26$ & UCHII & 2.73 & & -0.23 & & \\
\hline $35.58-0.03$ & UCHII & 1.57 & & -0.50 & & \\
\hline W49N & UCHII & 2.53 & & 0.38 & $\mathrm{Y}$ & \\
\hline W49S & UCHII & & & -0.63 & & \\
\hline $43.80-0.13$ & UCHII & 2.08 & & & & \\
\hline $45.07+0.13$ & UCHII & & -0.34 & -0.20 & $\mathrm{Y}$ & \\
\hline $59.78+0.06$ & UCHII & 2.62 & & & $\mathrm{Y}$ & $\mathrm{Y}$ \\
\hline S87 & UCHII & & & & $\mathrm{Y}$ & \\
\hline S88B & UCHII & 2.83 & -0.80 & -0.13 & $\mathrm{Y}$ & \\
\hline ON1 & UCHII & 2.04 & & -0.27 & & \\
\hline S106 & UCHII & 2.85 & -0.62 & 0.12 & & \\
\hline W75N & UCHII & 2.44 & -0.43 & -0.06 & $\mathrm{Y}$ & \\
\hline DR21S & UCHII & 2.70 & -0.30 & 0.38 & $\mathrm{Y}$ & $\mathrm{Y}$ \\
\hline CEPA & UCHII & & -0.46 & & $\mathrm{Y}$ & $\mathrm{Y}$ \\
\hline S158 & UCHII & 2.33 & -0.69 & & $\mathrm{Y}$ & $\mathrm{Y}$ \\
\hline $\operatorname{Mean}^{\mathrm{f}}$ & & $2.32(0.08)$ & $-0.48(0.06)$ & $-0.21(0.08)$ & & \\
\hline Median & & 2.35 & -0.45 & -0.20 & & \\
\hline $10.60-0.40$ & CHII & 2.61 & -0.38 & & & $\mathrm{Y}$ \\
\hline $12.21-0.10$ & CHII & 2.30 & & & & \\
\hline $19.61-0.23$ & CHII & 2.19 & & -0.33 & & \\
\hline W43S & CHII & 3.21 & -0.53 & -0.86 & & $\mathrm{Y}$ \\
\hline $32.80+0.20 \mathrm{AB}$ & CHII & & & -0.42 & & \\
\hline W44 & CHII & 2.33 & -0.18 & -0.23 & & $\mathrm{Y}$ \\
\hline $48.61+0.02$ & CHII & 3.42 & -1.05 & -1.16 & & \\
\hline M51M & CHII & 2.83 & -0.61 & -0.11 & & $\mathrm{Y}$ \\
\hline K3-50 & CHII & 2.53 & & & $\mathrm{Y}$ & \\
\hline ON2N & CHII & & & 0.06 & $\mathrm{Y}$ & \\
\hline S157 & CHII & 2.05 & -0.72 & -0.13 & & $\mathrm{Y}$ \\
\hline $\operatorname{Mean}^{\mathrm{f}}$ & & $2.61(0.18)$ & $-0.62(0.14)$ & $-0.40(0.15)$ & & \\
\hline Median & & 2.43 & -0.61 & -0.28 & & \\
\hline G123.07-6.3 & H II & 1.06 & -0.76 & -0.48 & $\mathrm{Y}$ & $\mathrm{Y}$ \\
\hline S235 & $\mathrm{H}_{\text {II }}$ & 1.72 & -0.66 & -0.35 & & \\
\hline $\mathrm{S} 252 \mathrm{~A}$ & $\mathrm{H}_{\text {II }}$ & 2.41 & -1.06 & -0.57 & & \\
\hline $13.87+0.28$ & H II & 2.80 & & -0.07 & & \\
\hline $23.95+0.16$ & $\mathrm{H}_{\text {II }}$ & 2.73 & & -0.21 & & $\mathrm{Y}$ \\
\hline
\end{tabular}


Table 13

(Continued)

\begin{tabular}{|c|c|c|c|c|c|c|}
\hline Source & $\mathrm{H}_{\mathrm{II}} ?^{\mathrm{a}}$ & $\begin{array}{c}\log \frac{L_{\mathrm{IR}}}{M_{\mathrm{vir}}\left(R_{\mathrm{CS} 5-4}\right)} \mathrm{b}, \mathrm{c} \\
\left(M_{\odot}\right)\end{array}$ & $\log \frac{L^{\prime}(\mathrm{HCN} 3-2)}{L^{\prime}(\mathrm{HCN} 1-0)} \mathrm{b}$ & $\log \frac{L^{\prime}(\mathrm{CS} 7-6)}{L^{\prime}(\mathrm{CS} 5-4)} b, \mathrm{c}$ & Outflow? ${ }^{\mathrm{d}}$ & Infall? ${ }^{\mathrm{e}}$ \\
\hline S76E & H II & 2.09 & & -0.31 & Y & \\
\hline $35.20-0.74$ & H II & 1.14 & & -0.21 & & \\
\hline W51W & H II & 2.89 & & 0.13 & & \\
\hline ON2S & H II & 2.62 & -0.21 & 0.17 & & \\
\hline Mean $^{\mathrm{f}}$ & & $2.30(0.22)$ & $-0.64(0.25)$ & $-0.21(0.08)$ & & \\
\hline Median & & 2.52 & -0.66 & -0.21 & & \\
\hline
\end{tabular}

Notes.

${ }^{a}$ The classification of evolutionary status of $\mathrm{H}$ II regions is judged from the size of the $\mathrm{H}$ II region from $2 \mathrm{~cm}$ survey. Refer to Shirley et al. (2003) and references in there for details.

${ }^{\mathrm{b}}$ Values are in $\log$ scale.

${ }^{c}$ CS 5-4 data from Shirley et al. (2003).

${ }^{\mathrm{d}}$ Refer to outflow catalogue, Wu et al. (2004).

e Judged from HCN 3-2 profile for $\delta v<-0.25$.

${ }^{\mathrm{f}}$ The mean in log scale. The uncertainty is the sigma of the mean.
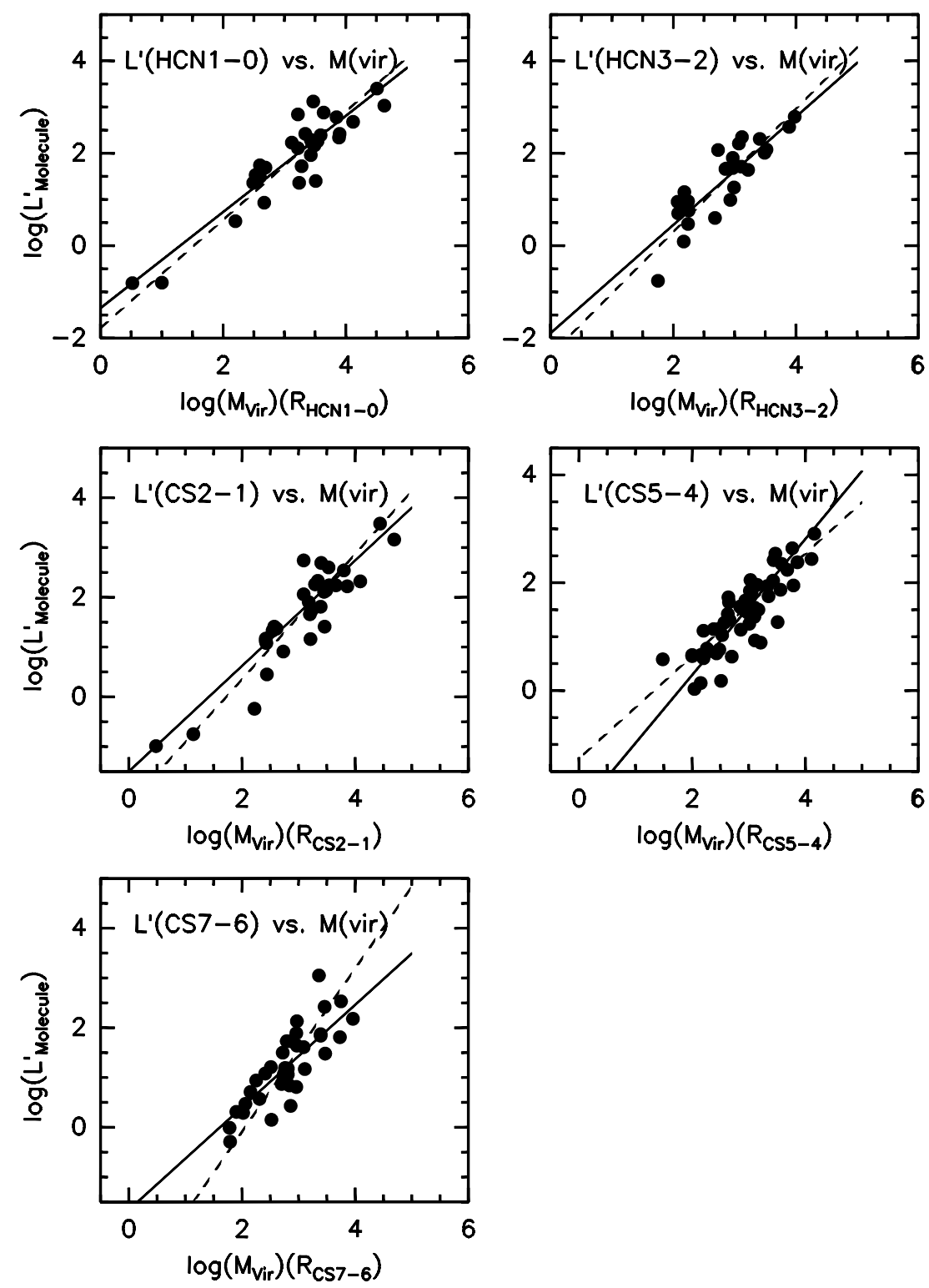

Figure 43. Correlation between the line luminosity of different transitions and the virial mass within FWHM of contour maps. The dashed line shows the linear least-squares fit with uncertainties; the solid line indicates the robust fit. Data of CS 5-4 come from Shirley et al. (2003). 

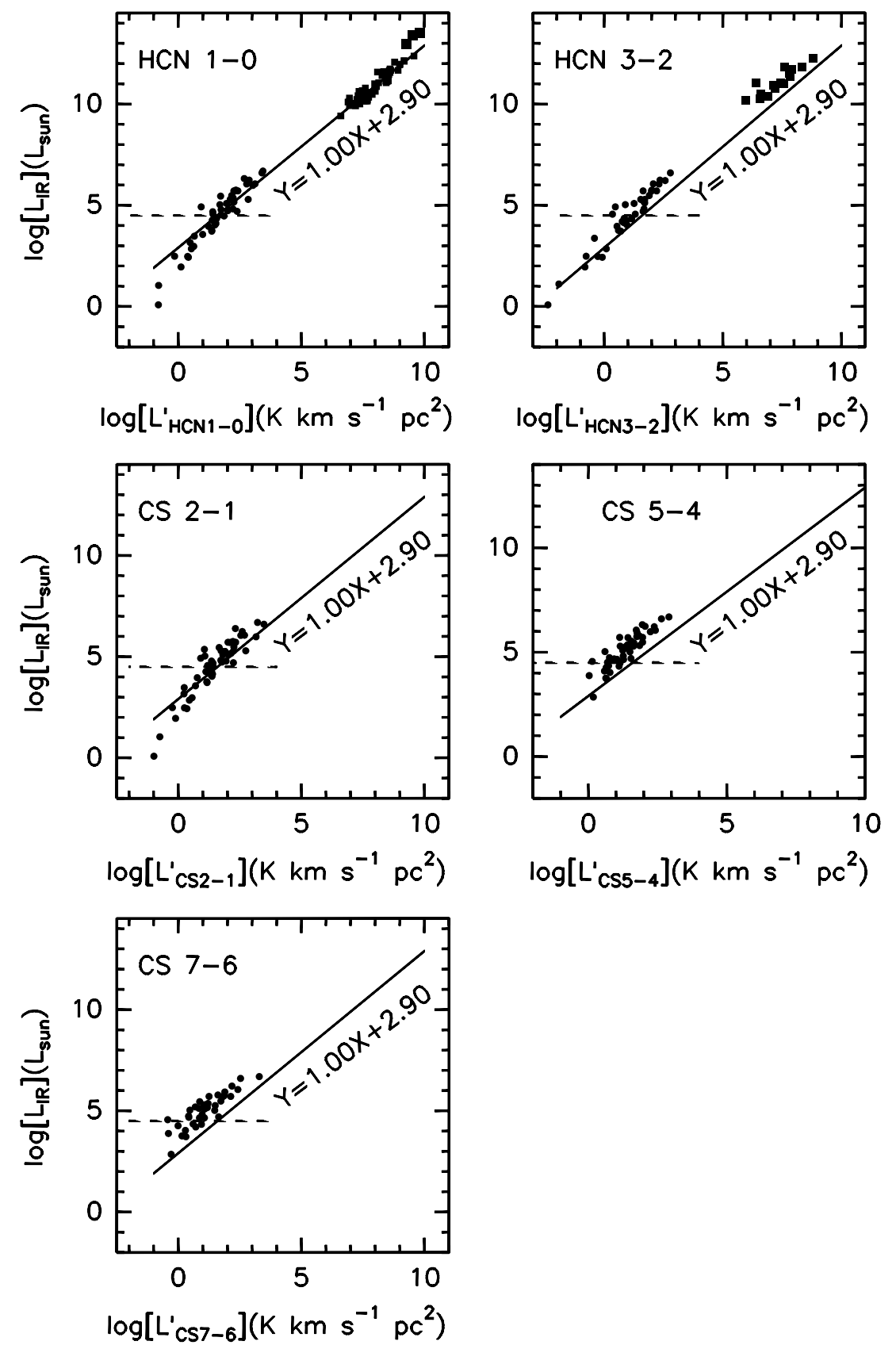

Figure 44. $L_{\mathrm{IR}}-L_{\mathrm{mol}}^{\prime}$ correlation for HCN 1-0, HCN 3-2, CS 2-1, CS 5-4, and CS 7-6 Galactic clumps. Data of CS 5-4 are taken from Shirley et al. (2003). The squares in the upper left panel are HCN 1-0 observations of galaxies (Gao \& Solomon 2004a) with three high- $z$ detections (Solomon et al. 2003; Vanden Bout et al. 2004; and Carilli et al. 2005). The squares in the upper right panel show the HCN 3-2 observations of galaxies (Bussmann et al. 2008). The solid line in the first panel shows the best-fit $L_{\mathrm{IR}}-L_{\mathrm{HCN} 1-0}^{\prime}$ correlation from galaxies, and is shown in other plots to indicate the correlation shifts between HCN $1-0$ and other tracers. The dashed line shows where $L_{\mathrm{IR}}=10^{4.5} L_{\odot}$.

Table 14

Statistics of Ratio $\frac{M_{\mathrm{Vir}}\left(R_{\text {molecule }}\right)}{L^{\prime}}$

\begin{tabular}{lcccc}
\hline \hline \multicolumn{1}{c}{ Ratio } & $\log \frac{M_{\mathrm{Vir}}\left(R_{\mathrm{CS} 2-1}\right)}{L_{\mathrm{CS} 2-1}^{\prime}}$ & $\log \frac{M_{\mathrm{Vir}}\left(R_{\mathrm{CS} 7-6}\right)}{L_{\mathrm{CS} 7-6}^{\prime}}$ & $\log \frac{M_{\mathrm{Vir}}\left(R_{\mathrm{HCN} 1-0}\right)}{L_{\mathrm{HCN} 1-0}^{\prime}}$ & $\log \frac{M_{\mathrm{Vir}}\left(R_{\mathrm{HCN} 3-2}\right)}{L_{\mathrm{HCN} 3-2}^{\prime}}$ \\
\hline Mean $^{\mathrm{a}}$ & $1.34 \pm 0.07$ & $1.57 \pm 0.07$ & $1.30 \pm 0.10$ & $1.35 \pm 0.07$ \\
Median & 1.30 & 1.59 & 1.20 & 1.29 \\
Std. dev. $^{\mathrm{b}}$ & 0.39 & 0.43 & 0.54 & 0.37 \\
\hline
\end{tabular}

Notes.

${ }^{\text {a }}$ The uncertainty is the sigma of the mean.

${ }^{b}$ The standard deviation of the ratios. 

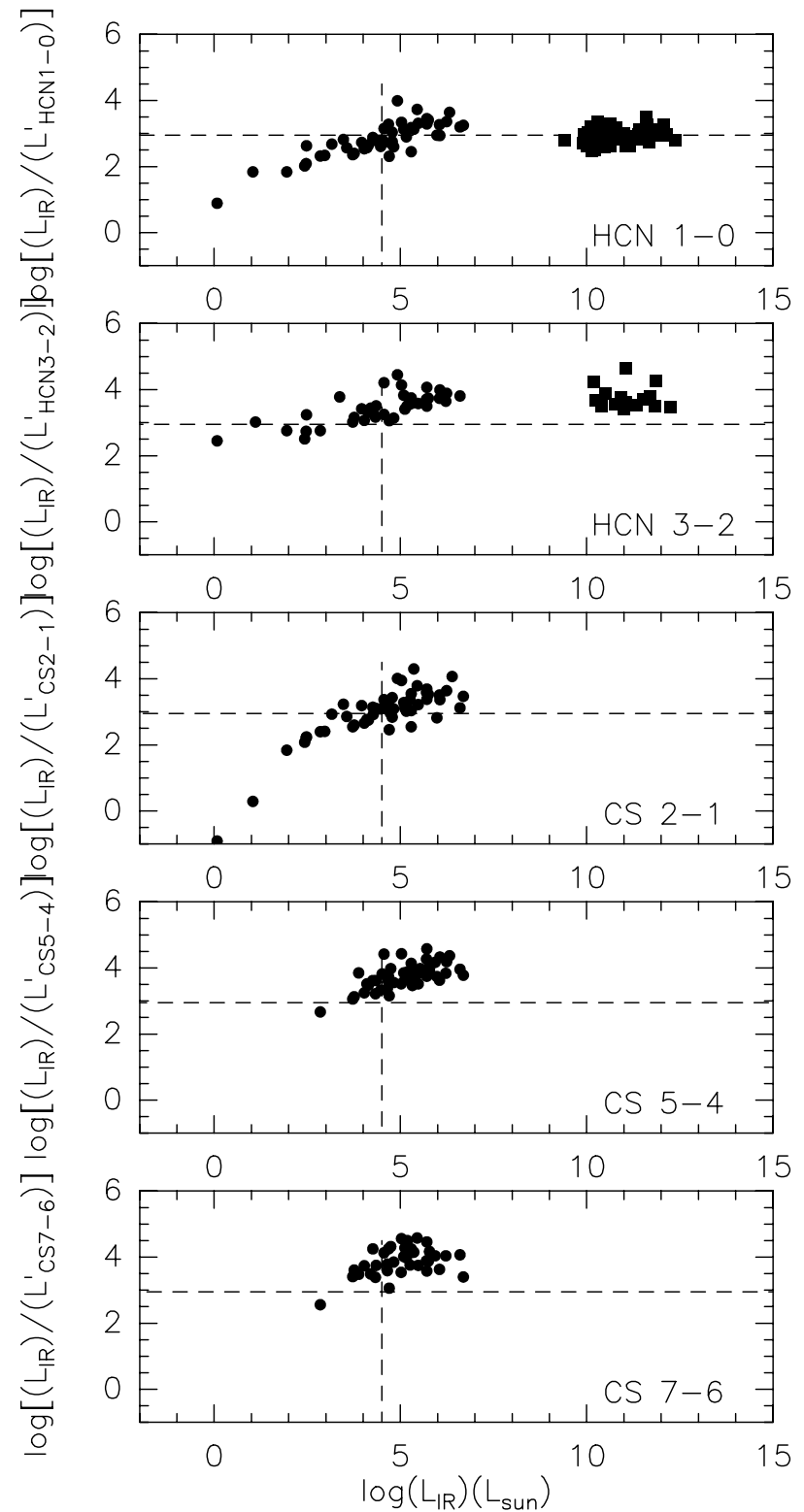

Figure 45. Correlations between the distant independent ratio $L_{\mathrm{IR}} / L_{\mathrm{mol}}^{\prime}$ and $L_{\mathrm{IR}}$ for different tracers. The squares in figures are $\mathrm{HCN} 1-0$ observations of galaxies (Gao \& Solomon 2004a) in the first panel, and HCN 3-2 observations of galaxies (Bussmann et al. 2008) in the second panel. The horizontal dashed line in the top plot indicates the averaged $L_{\mathrm{IR}} / L_{\mathrm{HCN} 1-0}^{\prime}$ ratio for galaxies; the vertical dashed line in the top plot shows the cutoff at $L_{\mathrm{IR}}=10^{4.5} L_{\mathrm{SUN}}$. These two lines are also shown in other plots to indicate the relative shifts of $L_{\mathrm{IR}} / L_{\mathrm{mol}}^{\prime}$ between $\mathrm{HCN} 1-0$ and other tracers.

\section{HCN 3-2:}

Least squares fit : $\log \left(L_{\mathrm{IR}}\right)=0.88( \pm 0.06) \times \log \left(L_{\mathrm{HCN} 3-2}^{\prime}\right)$

$$
+3.94( \pm 0.11) ; r=0.82
$$

Robust fit $: \log \left(L_{\mathrm{IR}}\right)=0.79 \times \log \left(L_{\mathrm{HCN} 3-2}^{\prime}\right)+4.09$

All the relations are close to linear. There is some tendency for the tracers with higher $n_{\text {crit }}$ to have slopes less than unity, as predicted by Narayanan et al. (2008). However they predict a slope of about 0.7 (ranges from $0.63 \sim 0.73$ from their Figure 7 ) for $\mathrm{HCN} 3-2$ whereas we see $0.88 \pm 0.06$. They do not predict values for CS transitions.
The ratio of $L_{\mathrm{IR}}$ to $L_{\mathrm{mol}}^{\prime}$ depends on the tracer. The constant term in the fit increases steadily as a function of excitation requirements. As discussed in Section 4.1, the luminosity of the higher- $J$ tracers is less for a given clump because of their higher requirements for both density and temperature. It is then natural that the relation depends on the tracer used. The dependence on critical density (or more generally on excitation requirements) is primarily in this constant term (which increases by an order of magnitude from CS 2-1 to CS 7-6) rather than in the slope in the $\log -\log$ plot.

\subsection{Comparing Explanations}

In order to explain the threshold and the linear correlation over a large range of scales, we have proposed a model of clustered star formation (Wu et al. 2005). We suggested that there is a basic unit of clustered formation. For $M_{\text {dense }}$ less than the mass of this unit, $L_{\mathrm{IR}} / M_{\text {dense }}$ rises rapidly with $M_{\text {dense }}$, as higher mass stars can form, and stellar luminosity rises rapidly with stellar mass. For $M_{\text {dense }}$ greater than the mass of this unit, the IMF is reasonably sampled and further increases in mass produce more units, but no further change in $L_{\mathrm{IR}} / M_{\text {dense }}$. If we suppose that larger scale cluster formation is built up by adding more and more such units, then the linear correlation between the total $L_{\mathrm{IR}}$ and $M_{\text {dense }}$ is a natural result, since $L_{\mathrm{HCN}}^{\prime}$ traces $M_{\text {dense }}$. In that case, the only difference between star formation on different scales and in different environments-from molecular clouds in our Galaxy to massive starbursts-is just how many such units they contain. Tables 15 and 16 contain the statistics of infrared luminosity, virial mass, and $L_{\mathrm{IR}} / \mathrm{M}_{\text {virial }}$ of all the massive dense clumps, and of the sample of massive dense clumps with $L_{\mathrm{IR}}>10^{4.5} L_{\odot}$ only, respectively.

The advantage of this model is that it can easily explain not only the linear correlation, but also the luminosity threshold: above a luminosity of about $10^{5} L_{\odot}$, the IMF is reasonably well sampled, and the dependence of stellar luminosity on stellar mass is less steep. The luminosity threshold is set by the requirement to sample the IMF. A threshold of $L_{\mathrm{IR}}>10^{4.5} L_{\odot}$ is consistent with a value $\left(\sim 10^{5} L_{\odot}\right)$ that dominates the luminosity function of the UCHII regions in the Milky Way (Casassus et al. 2000). This model can explain the fact that starburst galaxies have a very high star formation rate, but a similar star formation efficiency with respect to the dense gas as individual massive dense clumps in the Milky Way. Extreme starbursts in galaxies happen when entire molecular clouds become filled with dense gas, likely due to the huge pressure in the extreme environments.

To summarize, the model proposed by Wu et al. (2005) starts with what we know about well-studied regions of star formation in our Galaxy and tries to extend that picture to other galaxies.

The alternative models assume that star formation laws derived from extragalactic studies covering large scales can be extended to the scales where stars actually form. For example, Krumholz \& Thompson (2007) assumed that a Schmidt law in the form involving volume density describes star formation: $\dot{\rho}_{\star} \propto \rho_{g}^{1.5}$ over the full range of densities. They further assumed that the distribution of densities in a molecular cloud follows a lognormal probability distribution. They argued that a molecular tracer essentially measures the mass of gas with density equal to or greater than the critical density for that transition. With a simple model of radiative transfer to correct the critical density for trapping, they showed that these assumptions led to a linear relation between star formation rate and line luminosity of a transition when the mean density of the galaxy was less than the critical density of the transition, as seen for $\mathrm{HCN} 1-0$. 
Table 15

Statistics of Properties of Massive Dense Clumps ${ }^{\text {a }}$

\begin{tabular}{|c|c|c|c|c|c|}
\hline Satis. & $\begin{array}{c}\mathrm{M}_{\operatorname{vir}(\mathrm{HCN} 1-0)} \\
\left(M_{\odot}\right) \\
\end{array}$ & $\begin{array}{c}\mathrm{M}_{\mathrm{vir}(\mathrm{HCN} 3-2)} \\
\left(M_{\odot}\right) \\
\end{array}$ & $\begin{array}{c}\mathrm{M}_{\mathrm{vir}(\mathrm{CS} 2-1)} \\
\left(M_{\odot}\right) \\
\end{array}$ & $\begin{array}{c}\mathrm{M}_{\mathrm{vir}(\mathrm{CS} 7-6)} \\
\left(M_{\odot}\right) \\
\end{array}$ & $\begin{array}{c}L_{\mathrm{IR}} \\
\left(L_{\odot}\right) \\
\end{array}$ \\
\hline Mean $^{\text {b }}$ & $5300 \pm 1760$ & $1555 \pm 445$ & $5000 \pm 1870$ & $1300 \pm 310$ & $470000 \pm 120000$ \\
\hline Median & 2700 & 890 & 1950 & 650 & 106000 \\
\hline \multirow[t]{2}{*}{$\mathrm{Sdv}^{\mathrm{c}}$} & 9600 & 2310 & 10300 & 1930 & 920000 \\
\hline & $\begin{array}{c}\frac{L_{\mathrm{IR}}}{M_{\mathrm{vir}(\mathrm{HCN} 1-0)}} \\
\left(L_{\odot} / M_{\odot}\right)\end{array}$ & $\begin{array}{c}\frac{L_{\mathrm{IR}}}{M_{\mathrm{vir}(\mathrm{HCN} 3-2)}} \\
\left(L_{\odot} / M_{\odot}\right)\end{array}$ & $\begin{array}{c}\frac{L_{\mathrm{IR}}}{M_{\mathrm{vir}(\mathrm{CS} 2-1)}} \\
\left(L_{\odot} / M_{\odot}\right) \\
\end{array}$ & $\begin{array}{c}\frac{L_{\mathrm{IR}}}{M_{\mathrm{vir}(\mathrm{CS} 7-6)}} \\
\left(L_{\odot} / M_{\odot}\right) \\
\end{array}$ & \\
\hline Mean $^{\mathrm{b}}$ & $117 \pm 20$ & $371 \pm 102$ & $139 \pm 26$ & $592 \pm 195$ & \\
\hline Median & 98 & 182 & 129 & 229 & \\
\hline$S d v^{c}$ & 105 & 487 & 134 & 1085 & \\
\hline
\end{tabular}

Notes.

${ }^{a}$ Statistics on massive dense clumps only, which does not include the sources below the horizontal line in Table 1 .

$\mathrm{b}$ The uncertainty is the sigma of the mean.

c The standard deviation.

Table 16

Statistics of Properties of Massive Dense Clumps with $L_{\mathrm{IR}}>10^{4.5} L_{\odot}{ }^{\mathrm{a}}$

\begin{tabular}{|c|c|c|c|c|c|}
\hline Satis. & $\begin{array}{c}M_{\mathrm{vir}(\mathrm{HCN} 1-0)} \\
\left(M_{\odot}\right)\end{array}$ & $\begin{array}{c}M_{\mathrm{vir}(\mathrm{HCN} 3-2)} \\
\left(M_{\odot}\right)\end{array}$ & $\begin{array}{c}M_{\operatorname{vir}(\mathrm{CS} 2-1)} \\
\left(M_{\odot}\right)\end{array}$ & $\begin{array}{c}M_{\mathrm{vir}(\mathrm{CS} 7-6)} \\
\left(M_{\odot}\right)\end{array}$ & $\begin{array}{c}L_{\mathrm{IR}} \\
\left(L_{\odot}\right)\end{array}$ \\
\hline Mean $^{\mathrm{b}}$ & $6650 \pm 2350$ & $2070 \pm 630$ & $6300 \pm 2500$ & $1480 \pm 420$ & $630000 \pm 160000$ \\
\hline Median & 2950 & 1170 & 2500 & 710 & 200000 \\
\hline \multirow[t]{2}{*}{$\mathrm{Sdv}^{\mathrm{c}}$} & 10800 & 2600 & 11500 & 2030 & 1020000 \\
\hline & $\begin{array}{c}\frac{L_{\mathrm{IR}}}{M_{\mathrm{vir}(\mathrm{HCN} 1-0)}} \\
\left(L_{\odot} / M_{\odot}\right)\end{array}$ & $\begin{array}{c}\frac{L_{\mathrm{IR}}}{M_{\mathrm{vir}(\mathrm{HCN} 3-2)}} \\
\left(L_{\odot} / M_{\odot}\right)\end{array}$ & $\begin{array}{c}\frac{L_{\mathrm{IR}}}{M_{\mathrm{vir}(\mathrm{CS} 2-1)}} \\
\left(L_{\odot} / M_{\odot}\right)\end{array}$ & $\begin{array}{c}\frac{L_{\mathrm{IR}}}{M_{\mathrm{vir}(\mathrm{CS} 7-6)}} \\
\left(L_{\odot} / M_{\odot}\right)\end{array}$ & \\
\hline Mean $^{b}$ & $137 \pm 23$ & $473 \pm 130$ & $169 \pm 30$ & $726 \pm 246$ & \\
\hline Median & 120 & 324 & 155 & 439 & \\
\hline$S d v^{c}$ & 106 & 532 & 137 & 1204 & \\
\hline
\end{tabular}

Notes.

${ }^{a}$ Statistics on massive dense clumps only, which does not include the sources below the horizontal line in Table 1.

b The uncertainty is the sigma of the mean.

c The standard deviation.

If, on the other hand, the mean density of the gas $(\langle n\rangle)$ is greater than the critical density of the tracer, then the $L_{\mathrm{IR}}-L_{\text {tracer }}^{\prime}$ correlation will be super-linear, as is the case for CO. Narayanan et al. (2008) add to this picture the idea that index is flattened by the increasing index $(\beta)$ in the relation between $L_{\text {mol }}^{\prime}$ and density.

The average density determined from CS excitation of the massive clumps in our sample is about $10^{5.9} \mathrm{~cm}^{-3}$ (Plume et al. 1997), less than the critical density of all the tracers in this study except for the CS 2-1 line (Table 9), but greater than the effective density (Table 9) and the density that was found to contribute most to the HCN 1-0 line in the simulations of Krumholz \& Thompson (2007). In fact, a density derived from excitation analysis is biased toward the densest regions and the mean density of the clumps in the sense of mass divided by volume is generally less (e.g., Shirley et al. 2003). As noted above, the relations we find do not support the suggestions by Krumholz \& Thompson (2007) or Narayanan et al. (2008).

Another problem with the Krumholz-Thompson argument is that there is no evidence in our Galaxy that the star formation density depends on the local gas density in the way assumed for a Schmidt law. Rather, there is a threshold density below which there is essentially no star formation. Above that density, clumps and cores do not have lognormal distributions, but instead have centrally condensed power-law distributions (Mueller et al. 2002; Shirley et al. 2003).
More detailed discussion about the origin of the $L_{\mathrm{IR}}-L_{\text {tracer }}^{\prime}$ correlation is beyond the scope of this paper. More discussion can be found in recent reviews and papers (e.g., Shirley et al. 2008; Narayanan et al. 2008; Evans 2008; Baan et al. 2008; Iono et al. 2009; Juneau et al. 2009).

\subsection{Caveats: Different Beams and Different Distances}

The beam sizes used to map the lower excitation lines were roughly twice as large as those used for the higher excitation lines (Table 2). As shown by Liszt \& Linke (1975), derivation of densities via excitation analysis with such data can be very misleading. Densities from such an analysis with better-matched beams have been obtained for this sample by Plume et al. (1997), and we have not analyzed the current data in that way. Instead, we focused on the new feature of these data, which are maps of multiple transitions. We treated each transition separately and derived mean surface and volume densities for each tracer (Section 4.4). We then discussed the difference between these mean densities and those derived by Plume et al. (1997) from excitation analysis.

The sources in this study lie at a large range of distances $(0.2$ to $13.7 \mathrm{kpc})$. This is an inevitable consequence of our attempt to capture the full range of properties of massive dense clumps, as many of the most extreme star-forming clumps are at large distances. The effective linear resolution is much worse for the distant clumps, which could bias the determinations 

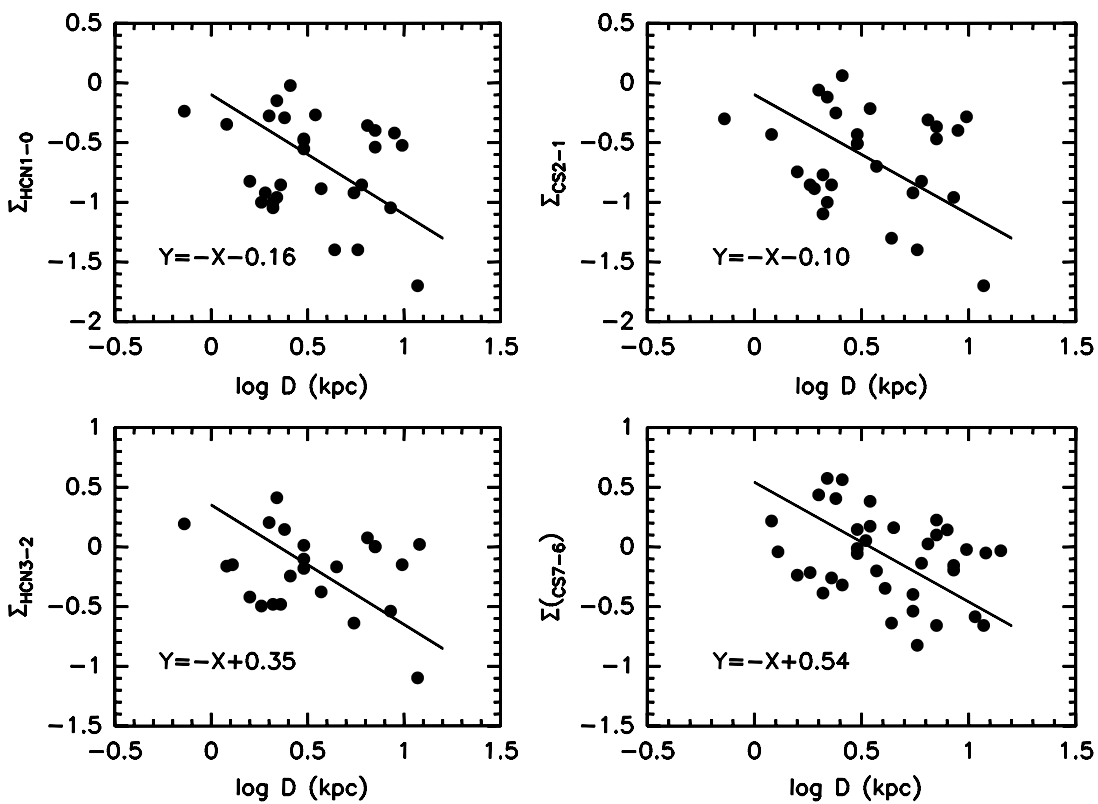

Figure 46. Log-log scale plots of the surface density vs. distance for massive clumps for different transitions. The lines in the plots have fixed slopes of -1 with different offsets.
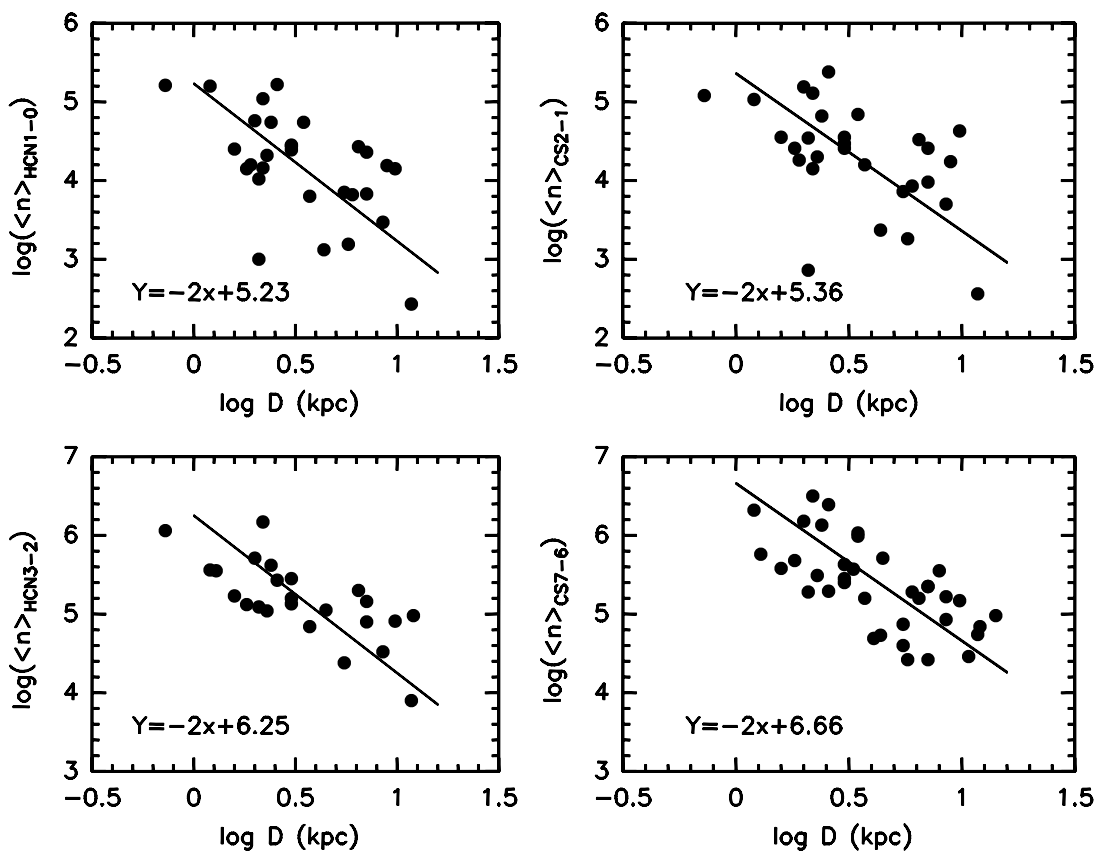

Figure 47. Log-log scale plots of the averaged volume density vs. distance for massive clumps with different transitions. The lines in the plots have fixed slopes of -2 with different offsets.

of some quantities, such as surface and volume densities, to lower values. Examination of Equations (3), (5), and (6) would suggest that $\Sigma \propto d^{-1}$ and $\bar{n} \propto d^{-2}$ if there were no trends in mass with distance. To check the importance of this effect, we plot in Figures 46 and 47 the logarithms of surface and mean volume densities versus the logarithm of distance. The lines in the plots have slopes fixed to the values suggested above and only the offset is fitted. With substantial scatter, the expected relations are seen. Note that these relations assume that there is no distance effect on the clump masses. Sensitivity limits might lead to more distant clouds being systematically more massive, partly canceling the effects of decreasing resolution.

We would expect no strong trends with distance in line ratio plots, and indeed none are seen (Figure 48).

\section{SUMMARY}

Maps of over 50 massive, dense clumps in HCN and CS were obtained. The main sample was drawn from the sample of Plume et al. (1992) selected from water maser surveys and found to have CS 7-6 emission. In addition, we mapped a smaller number of sources of lower luminosity from other surveys. These are not included in statistics quoted for massive, dense clumps. We have presented maps of $\mathrm{HCN} 1-0$ and $3-2$, along with CS 2-1 and 7-6. Together with previously published maps of CS 5-4, these provide the basis for analysis of systematics of massive, dense clumps in tracers with a wide range of excitation requirements. Upper state energies in temperature units range from $4.3 \mathrm{~K}$ to $66 \mathrm{~K}$, critical densities range from 

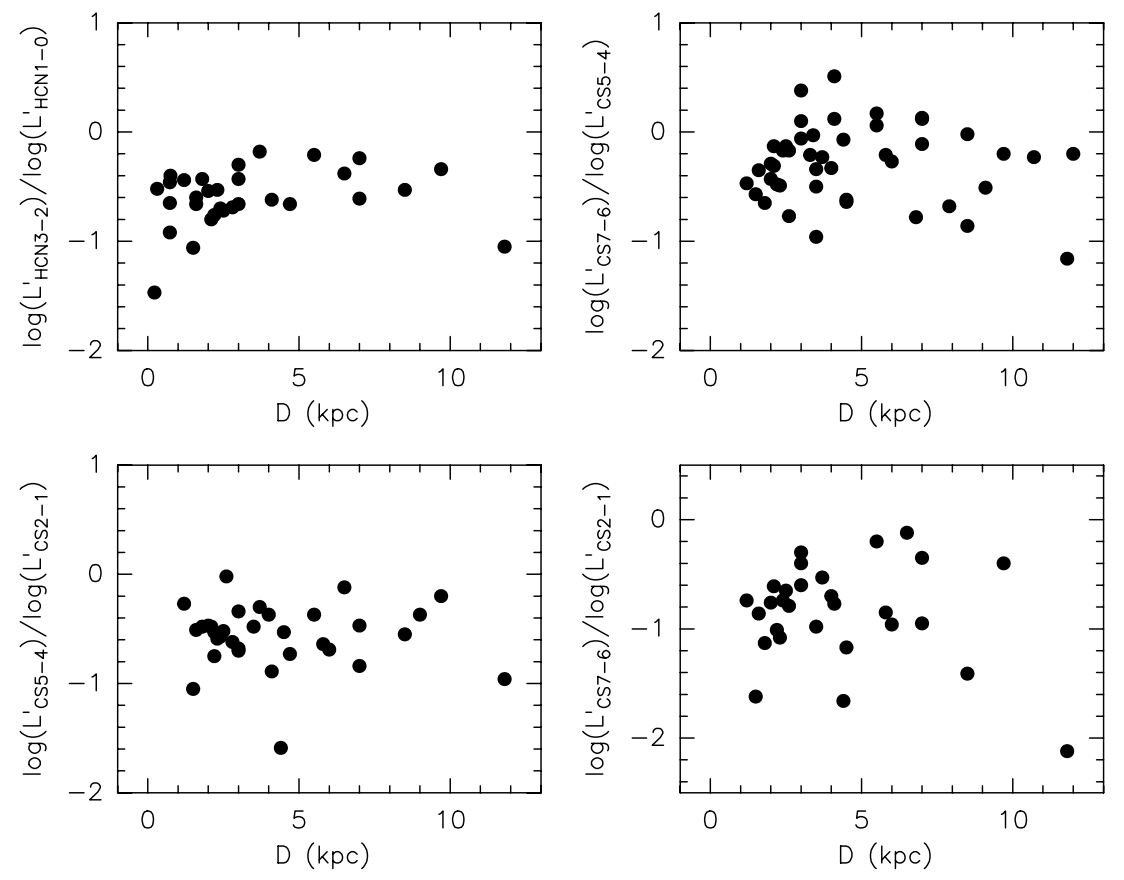

Figure 48. Plots of the line ratios vs. distance for massive clumps. No obvious trends are seen.

$3.9 \times 10^{5} \mathrm{~cm}^{-3}$ to $6.8 \times 10^{7} \mathrm{~cm}^{-3}$, and effective densities range from $4.1 \times 10^{3} \mathrm{~cm}^{-3}$ to $2.6 \times 10^{5} \mathrm{~cm}^{-3}$.

As excitation requirements increase, FWHM map sizes shrink, as expected for centrally peaked density distributions. Interestingly, the linewidths increase with excitation requirement, producing an inverse size-linewidth relation, opposite to the one found for low density regions. The linewidths are highly supersonic, with Mach numbers greater than 6. These facts suggest that outflows and expanding $\mathrm{H}$ II regions are injecting turbulence on small scales. An alternative would be increased inflow velocities.

Virial masses were computed for each of the tracers. Because the tracers with higher excitation requirements have smaller FWHM sizes, the virial masses derived from them are less. For the tracers that best trace the whole clump, CS $2-1$ and HCN 1-0, the mean and median virial masses are 5000 and $1950 M_{\odot}(\mathrm{CS})$, or 5300 and $2700 M_{\odot}(\mathrm{HCN})$. The mean and median infrared luminosities are $4.7 \times 10^{5} L_{\odot}$ and $1.06 \times 10^{5} L_{\odot}$. These clumps include some of the most massive and luminous star-forming regions in the Galaxy, such as W43, W51, and W49. For clumps with luminosity above $10^{4.5} L_{\odot},\left\langle L_{\mathrm{IR}}\right\rangle=(6.3 \pm 1.6) \times 10^{5} L_{\odot}$.

Mass surface densities are similar to those needed for high-mass star formation, according to theoretical arguments (Krumholz \& McKee 2008). Even though only CS 7-6 indicates a mean surface density exceeding their threshold of $1 \mathrm{~g} \mathrm{~cm}^{-2}$ for massive star formation, the observational and theoretical uncertainties are sufficient to suggest that most of these clumps can form massive stars. And their high luminosities indicate that indeed they are doing so.

Line profiles skewed to the blue, using the blue peak criterion, are common (16 examples) in the sample, but some (nine) are skewed to the red. This excess of blue over red is less than in the original sample of Wu \& Evans (2003), but it still suggests the existence of large scale inflow motions in many clumps.

The line luminosities all correlate very well with virial mass, with different offsets because of the fact that higher excitation transitions trace smaller fractions of the core. There is no apparent correlation of line luminosity with surface density or volume density, contrary to some recent suggestions (e.g., Narayanan et al. 2008). Line ratios show little correlation with any other properties. In principle, ratios of line luminosity should trace density, but in a power-law density distribution, densities range widely and line ratios tend to constant values.

The far-infrared luminosity correlates very well with all the line luminosities. The correlations are close to linear, with some marginal evidence for lower exponents for lines with the most stringent excitation requirements. While that agrees qualitatively with predictions by Narayanan et al. (2008), the effect is much less than the predicted one.

Overall, the data support the idea that the linear relation between $L_{\mathrm{IR}}$ and $L_{\mathrm{mol}}^{\prime}$ for starburst galaxies and molecular lines that are hard to excite can be explained if such galaxies have large numbers of massive, dense clumps similar to those in this study. In this case, the star formation rate in starburst galaxies depends on the total mass of gas above the threshold density or surface density needed for massive star formation, rather than on a local, nonlinear form of the Schmidt law.

We thank D. Narayanan and M. Krumholz for lively discussions that illuminated their models. J.W. thanks the postdoctoral fellowship support from the Submillimeter Array of the Smithsonian Observatory while completing this work. This work was supported in part by NSF Grant AST-0607793 and by McDonald Observatory.

\section{REFERENCES}

Aguirre, J., et al. 2009, ApJS, submitted

Ahrens, V., Lewen, F., Takano, S., Winnewisser, G., Urban, S., Negirev, A. A., \& Koroliev, A. N. 2002, Z. Naturforsch., 57a, 669

Anglada, G., Estalella, R., Pastor, J., Rodriguez, L. F., \& Haschick, A. D. 1996, ApJ, 463, 205

Barranco, J. A., \& Goodman, A. A. 1998, ApJ, 504, 207

Baan, W. A., Henkel, C., Loenen, A. F., Baudry, A., \& Wiklind, T. 2008, A\&A, 447,747

Bertoldi, F., \& McKee, C. F. 1992, ApJ, 395, 140

Beuther, H., Schilke, P., Menten, K. M., Motte, F., Sridharan, T. K., \& Wyrowski, F. 2002, ApJ, 566, 945 
Blitz, L., Fich, M., \& Stark, A. A. 1982, ApJS, 49, 183

Bonnell, I. A., \& Bate, M. R. 2005, MNRAS, 362, 915

Bonnell, I. A., Bate, M. R., Clarke, C. J., \& Pringle, J. E. 1997, MNRAS, 285, 201

Bonnell, I. A., Bate, M. R., \& Zinnecker, H. 1998, MNRAS, 298, 93

Brand, J., \& Blitz, L. 1993, A\&A, 275, 67

Braz, M. A., \& Epchtein, N. 1983, A\&A, 54, 167

Bussmann, R. S., et al. 2008, ApJ, 681, L73

Calzetti, D. 2008, in ASP Conf. Ser. 390, Pathways Through an Eclectic Universe, ed. J. H. Knapen, T. J. Mahoney, \& A. Vazdekis (San Francisco, CA: ASP), 121

Caselli, P., \& Myers, P. C. 1995, ApJ, 446, 665

Carilli, C. L., et al. 2005, ApJ, 618, 586

Carpenter, J. M. 2000, AJ, 120, 3139

Casassus, S., Bronfman, L., May, J., \& Nyman, L. -A. 2000, A\&A, 358, 514

Cernis, K. 1990, Ap\&SS, 166, 315

Chen, H., Myers, P. C., Ladd, E. F., \& Wood, D. O. S. 1995, ApJ, 445, 377

Chini, R., Kreysa, E., Mezger, P. G., \& Gemuend, H. -P. 1986, A\&A, 154, L8

Churchwell, E., Wolfire, M. G., \& Wood, D. O. S. 1990, ApJ, 354, 247

Dame, T. M., \& Thaddeus, P. 1985, ApJ, 297, 751

Downes, D., Wilson, T. L., Bieging, J., \& Wink, J. 1980, A\&A, 91, 186

Elmegreen, B. G. 1985, in Protostar and Planets II, ed. D. C. Black \& M. S. Matthews (Tucson, AZ: Univ. Arizona Press), 33

Evans, N. J., II. 1989, RevMexAA, 18, 21

Evans, N. J., II. 1999, Ann. Rev. Ast. \& Astrophys., 37, 311

Evans, N. J., II. 2003, in Proc. Conf. on Chemistry as a Diagnostic of Star Formation, University of Waterloo, Canada, 2002 August 21-23, ed. C. L. Curry \& M. Fich (Ottawa: NRC Press), 157

Evans, N. J., II. 2008, in ASP Conf. Ser. 390, Pathways Through an Eclectic Universe, ed. J. H. Knapen, T. J. Mahoney, \& A. Vazdekis (San Francisco, CA: ASP), 52

Fuller, G. A., Williams, S. J., \& Sridharan, T. K. 2005, A\&A, 442, 949

Gao, Y., \& Solomon, P. M. 2004a, ApJ, 606, 271

Gao, Y., \& Solomon, P. M. 2004b, ApJS, 152, 63

Genzel, R., \& Downes, D. 1977, A\&AS, 30, 145

Goodman, A. A., Barranco, J. A., Wilner, D. J., \& Heyer, M. H. 1998, ApJ, 504, 223

Gottlieb, C. A., Myers, P. C., \& Thaddeus, P. 2003, ApJ, 588, 655

Gregersen, E. M., \& Evans, N. J., II. 2000, ApJ, 538, 260

Gregersen, E. M., Evans, N. J., II, Mardones, D., \& Myers, P. C. 2000, ApJ, 533, 440 .

Gregersen, E. M., Evans, N. J., II, Zhou, S., \& Choi, M. 1997, ApJ, 484, 256

Harris, S., \& Wynn-Williams, C. G. 1976, MNRAS, 174, 649

Hofner, P., Kurtz, S., Churchwell, E., Walmsley, C. M., \& Cesaroni, R. 1996, ApJ, 460, 359

Hunter, T. R., Churchwell, E., Watson, C., Cox, P., Benford, D. J., \& Roelfsema, P. R. 2000, AJ, 119, 2711

Iono, D., et al. 2009, ApJ, 695, 1537

Juneau, S., Narayanan, D. T., Moustakas, J., Shirley, Y. L., Bussmann, R. S., Kennicutt, R. C., \& Vanden Bout, P. A. 2009, ApJ, 707, 1217

Kauffmann, J., Bertoldi, F., Bourke, T. L., Evans, N. J., II, \& Lee, C. W. 2008, A\&A, 487, 993

Kennicutt, R. C. 1998, ApJ, 498, 541

Kennicutt, R. C., et al. 2007, ApJ, 671, 333

Keto, E. R. 1990, ApJ, 355, 190

Krumholz, M. R., \& Bonnell, I. A. 2007, arXiv:0712.0828

Krumholz, M. R., Cunningham, A. J., Klein, R. I., \& McKee, C. F. 2010, arXiv:1001.0971

Krumholz, M. R., Klein, R. I., \& McKee, C. F. 2007, ApJ, 656, 959

Krumholz, M. R., \& McKee, C. F. 2008, Nature, 451, 1082

Krumholz, M. R., \& Thompson, T. A. 2007, ApJ, 669, 289

Kun, M., \& Prusti, T. 1993, A\&A, 272, 235

Kurtz, S. 2002, in ASP Conf. Ser. 267, Hot Star Workshop III: The Earliest Phases of Massive Star Birth, ed. P. A. Crowther (San Francisco, CA: ASP), 81

Larson, R. B. 1981, MNRAS, 194, 809

Lada, C. J., \& Lada, E. A. 2003, ARA\&A, 41, 57
Li, D., Velusamy, T., Goldsmith, P. F., \& Langer, W. D. 2007, ApJ, 655, 351

Liszt, H. S., \& Linke, R. A. 1975, ApJ, 196, 709

McKee, C. F., \& Tan, J. C. 2002, Nature, 416, 59

McKee, C. F., \& Tan, J. C. 2003, ApJ, 585, 850

Mueller, K. E., Shirley, Y. L., Evans, N. J., II, \& Jacobson, H. R. 2002, ApJS, 143,469

Mundy, L. G., Evans, N. J., II, Snell, R. L., Goldsmith, P. F., \& Bally, J. 1986, ApJ, 306, 670

Mundy, L. G., Evans, N. J., II, Snell, R. L., \& Goldsmith, P. F. 1987, ApJ, 318, 392

Myers, P. C. 1985, in Protostars and Planets II, ed. D. C. Black \& M. S. Matthews (Tucson, AZ: Univ. Arizona Press), 81

Narayanan, D., Cox, T. J., Shirley, Y., Dave, R., Hernquist, L., \& Walker, C. K. 2008, ApJ, 684, 996

O’Dell, C. R. 2005, Book Review, The Orion Nebula: Where Stars are Born (Cambridge, MA: Harvard Univ. Press), 170

Pirogov, L., Lapinov, A., Zinchenko, I., \& Shul'Ga, V. 1996, Astron. Astrophys. Trans., 11, 287

Pirogov, L. 1999, A\&A, 348, 600

Plume, R., Jaffe, D. T., \& Evans, N. J., II. 1992, ApJS, 78, 505

Plume, R., Jaffe, D. T., Evans, N. J., II, Martin-Pintado, J., \& Gomez-Gonzalez, J. 1997, ApJ, 476, 730

Price, S. D., Egan, M. P., Carey, S. J., Mizuno, D. R., \& Kuchar, T. A. 2001, AJ, 121,2819

Rosolowsky, E., et al. 2009, arXiv:0909.2871

Sanders, D. B., \& Mirabel, I. F. 1996, ARA\&A, 34, 749

Schmidt, M. 1959, ApJ, 129, 243

Schuller, F., et al. 2009, A\&A, 504, 415

Shirley, Y. L., Evans, N. J., II, Mueller, K. E., Knez, C., \& Jaffe, D. T. 2003, ApJS, 149,375

Shirley, Y. L., Wu, J., Bussmann, R. S., \& Wootten, A. 2008, in ASP Conf. Ser. 387, Massive Star Formation: Observations Confront Theory, ed. H. Beuther, H. Linz, \& T. Henning (San Francisco, CA: ASP), 401

Snell, R. L., Goldsmith, P. F., Erickson, N. R., Mundy, L. G., \& Evans, N. J., II. 1984, ApJ, 276, 625

Solomon, P. M., Rivolo, A. R., Barret, J., \& Yahil, A. 1987, ApJ, 319, 370

Solomon, P. M., Vanden Bout, P. A., Carilli, C. L., \& Guelin, M. 2003, Nature, 356,318

Sridharan, T. K., Beuther, H., Schilke, P., Menten, K. M., \& Wyrowski, F. 2002, ApJ, 566, 931

Tan, J. C., Krumholz, M. R., \& McKee, C. F. 2006, ApJ, 641, L121

Urban, A., Martel, H., \& Evans, N. J. 2010, ApJ, 710, 1343

Val'tts, I. E., Ellingsen, S. P., Slysh, V. I., Kalenskii, S. V., Otrupcek, R., \& Larionov, G. M. 2000, MNRAS, 317, 315

Vanden Bout, P. A., Solomon, P. M., \& Maddalena, R. J. 2004, ApJ, 614, 97

Velusamy, T., Peng, R., Li, D., Goldsmith, P. F., \& Langer, W. D. 2008, ApJ, 688, L87

Williams, J. P., Blitz, L., \& McKee, C. F. 2000, in Protostar and Planets IV, ed. V. Mannings, A. P. Boss, \& S. S. Russell (Tucson, AZ: Univ. Arizona Press), 97

Williams, J. P., et al. 2007, BAAS, 38, 110

Wink, J. E., Altenhoff, W. J., \& Mezger, P. G. 1982, A\&A, 108, 227

Wood, D. O. S., \& Churchwell, E. 1989, ApJ, 340, 265

Wu, J., \& Evans, N. J., II. 2003, ApJ, 592, L79

Wu, J., Evans, N. J., II, Gao, Y., Solomon, P., Shirley, Y., \& Vanden Bout, P. A. 2005, ApJ, 635, L173

Wu, J., Vanden Bout, P. A., Evans, N. J., II., \& Dunham, M. M. 2009, ApJ, 707, 988

Wu, Y., Wei, Y., Zhao, M., Shi, Y., Yu, W., Qin, S., \& Huang, M. 2004, A\&A, 426, 503

Zhang, Q., Hunter, T. R., Brand, J., Sridharan, T. K., Cesaroni, R., Molinari, S., Wang, J., \& Kramer, M. 2005, ApJ, 625, 864

Zhou, S., Evans, N. J., \& Wang, Y. 1996, ApJ, 406, 296

Zinchenko, I., Forsstroem, V., Lapinov, A., \& Mattila, K. 1994, A\&A, 288, 601

Zinchenko, I., Mattila, K., \& Toriseva, M. 1995, A\&AS, 111, 95 